\title{
Having Fun, Working Out: Adaptive and Engaging Video Games for Exercise
}

by

Christopher Burt

A thesis submitted to the Faculty of Graduate and Post-Doctoral Affairs

in partial fulfillment of the requirements for the degree of

\author{
Master of Applied Science \\ in \\ Human-Computer Interaction
}

Carleton University

Ottawa, Ontario

(C) 2014

Christopher Burt 



\section{Abstract}

This study evaluates an original game, Sea Monkeys, which contributes to the field of adaptive games while testing the viability of exergames that present continuous, expandable narrative. Adaptivity stands to assist game designers in resolving challenge management, a sophisticated problem in exergaming. Psychological flow provides a unified perspective from which to evaluate experiences of physical exertion and game engagement. The study was performed using independent measures to discern the effect of adaptivity ( $\mathrm{n}=25)$. Participants were exposed to two rounds of the game using full-body motion input and equipped with a heart rate monitor. Cardiovascular exertion meeting exercise standards was observed. Measurements suggest that adaptivity enhanced the reliability of the challenge encountered by players. Players reported the narrative to be engaging and helpful, and flow experiences were indicated. Sea Monkeys demonstrates an engaging, effective, and expandable game design for delivering exercise benefit. 


\section{Acknowledgements}

Thanks to my family for endless support. My gratitude goes out to my friends who helped me along the way. My best wishes go to Dennis Arsenault, my exergaming comrade, and Shannon Fenton, who created the game's charming characters.

I extend my gratitude to my supervisor, Anthony Whitehead, for providing generous and exciting opportunities throughout my education, and dynamic and enthusiastic guidance throughout my efforts.

Thanks also to my defense committee for the opportunity to present what I've learned. 


\section{Contents}

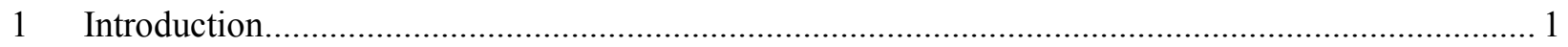

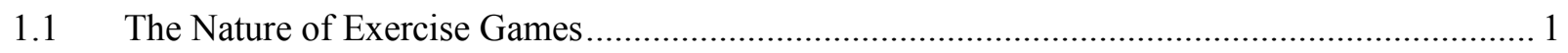

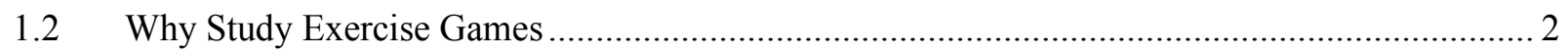

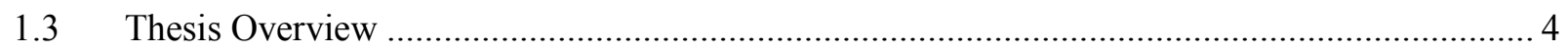

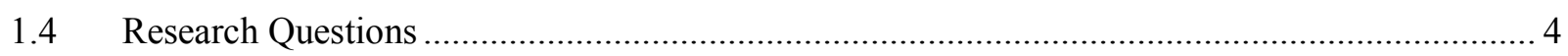

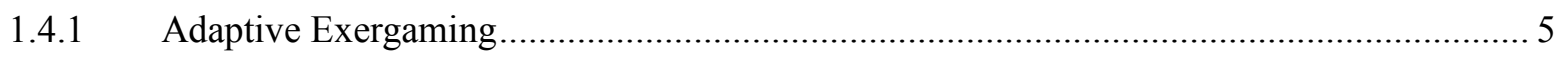

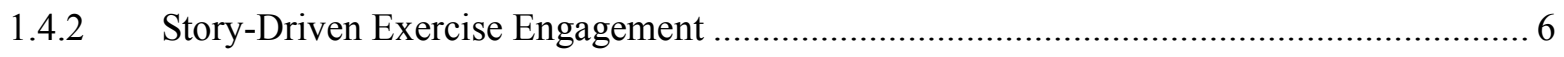

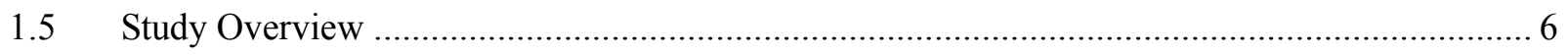

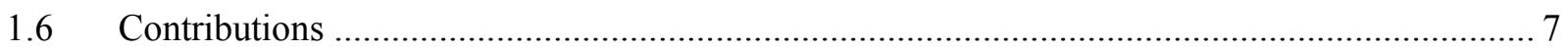

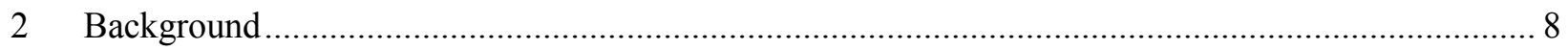

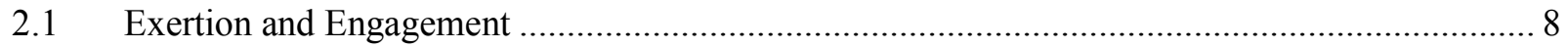

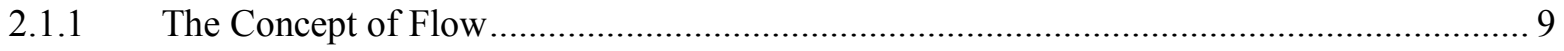

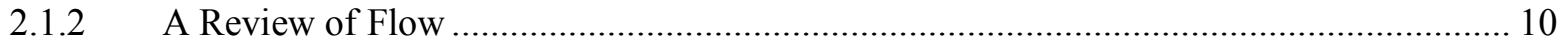

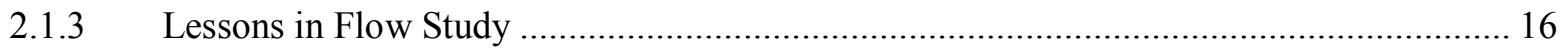

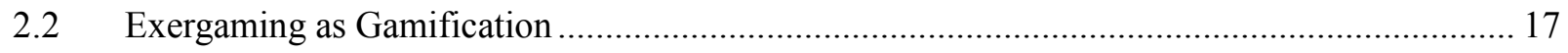

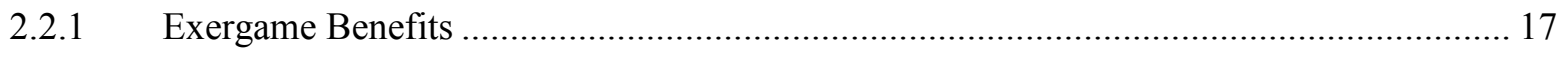

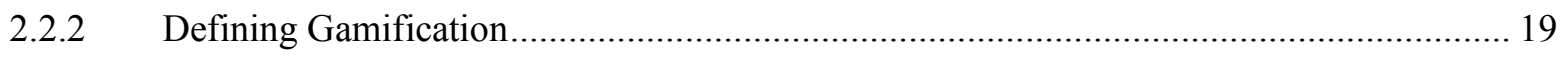

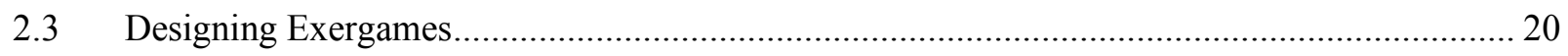

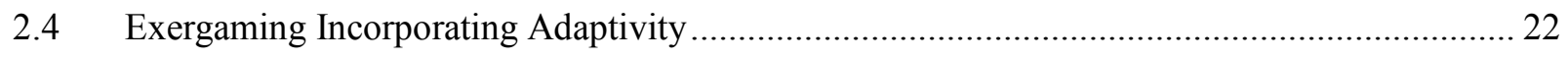

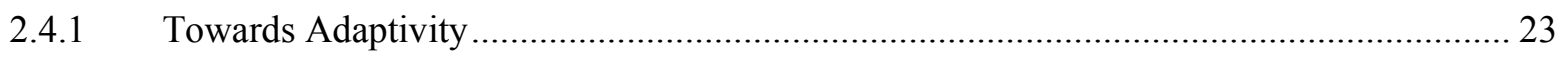

2.4.2 A Framework for Adaptive Exergames ................................................................... 24

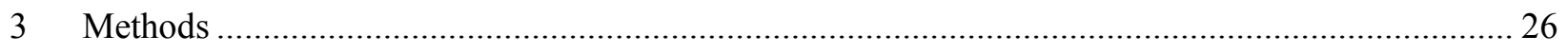

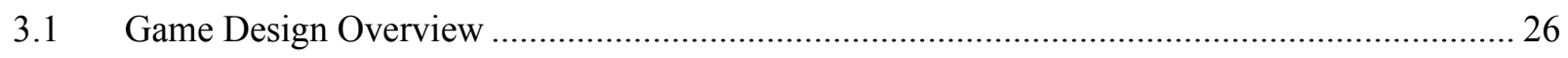

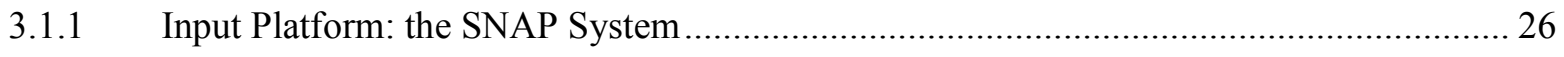




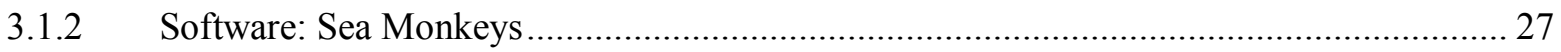

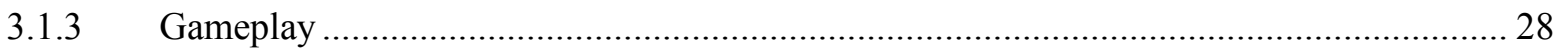

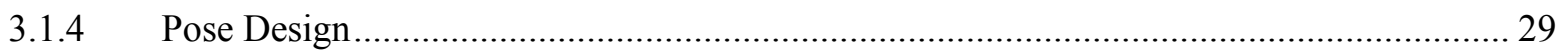

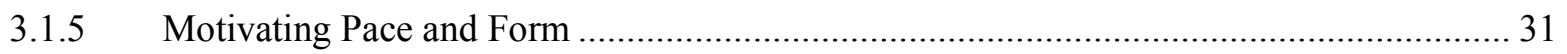

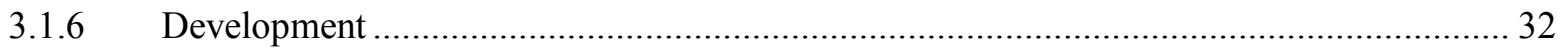

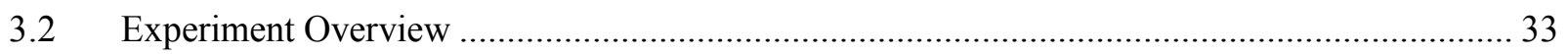

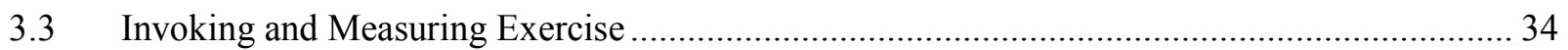

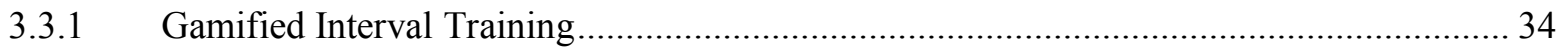

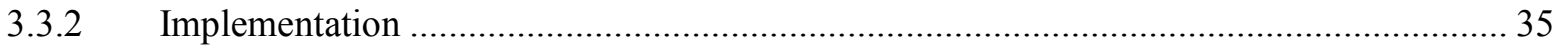

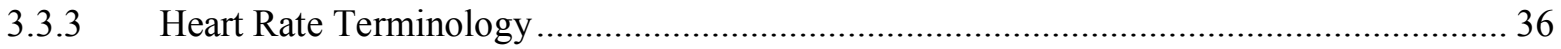

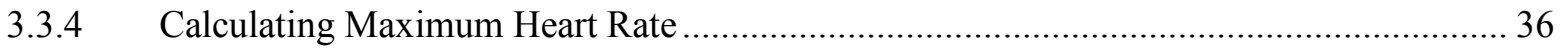

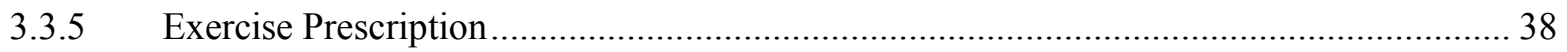

3.3.6 Invoking and Measuring Exercise: Evaluation ........................................................ 38

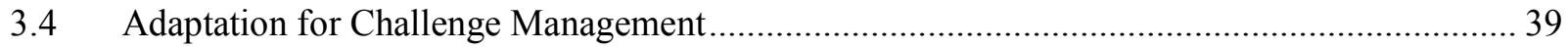

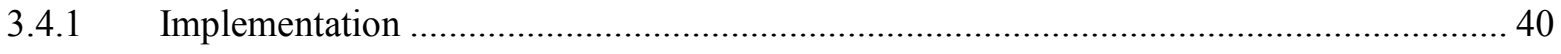

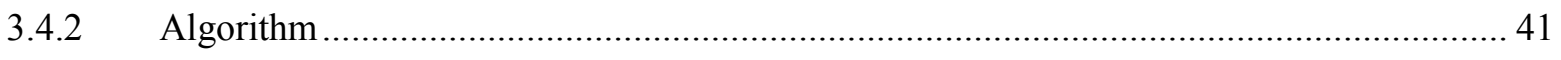

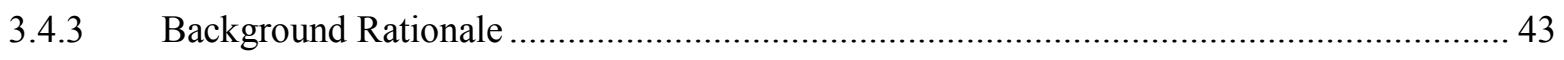

3.4.4 Adaptation for Challenge Management: Evaluation .................................................. 44

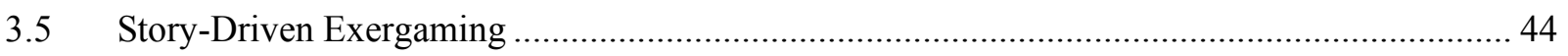

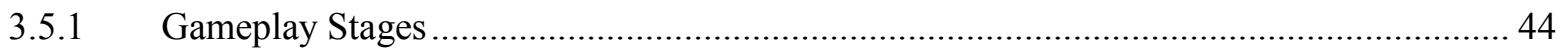

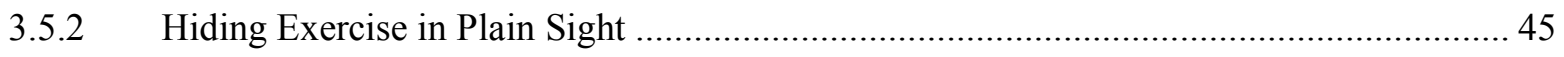

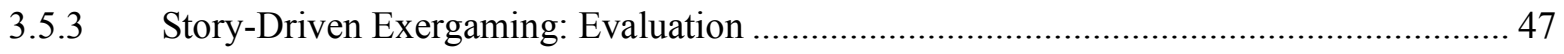

3.6 Fulfilling Design Requirements and Addressing Limitations ................................................ 48

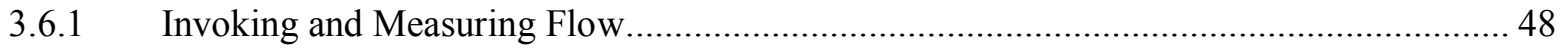

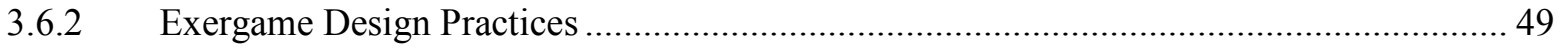

3.6.3 Gathering Robust Experiential Reports .............................................................. 50 


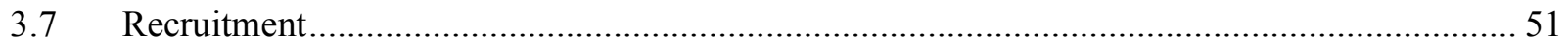

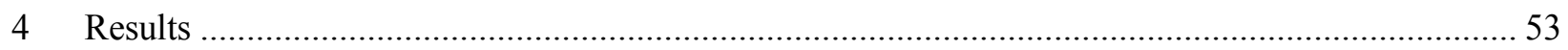

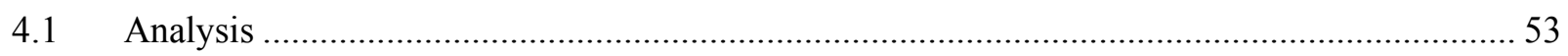

4.1.1 Adaptation is Suggested to Increase Workout Reliability ............................................ 53

4.1.2 Story-Driven Exergaming Can Promote Flow and Enjoyment..................................... 56

4.1.3 Verification of Interval Training Intensity ............................................................ 58

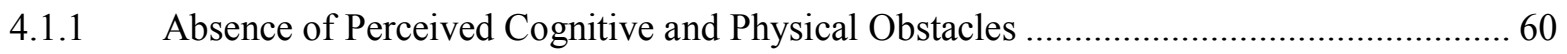

4.1.2 Correlations with Motion Gaming Experience ................................................................ 62

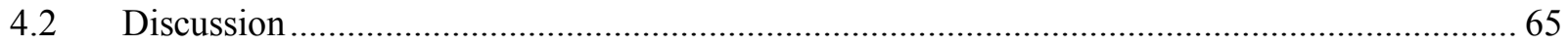

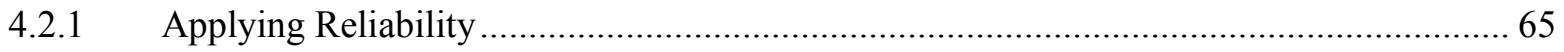

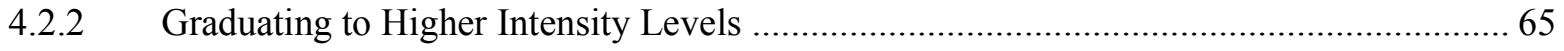

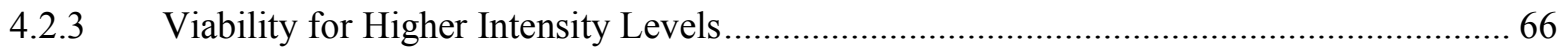

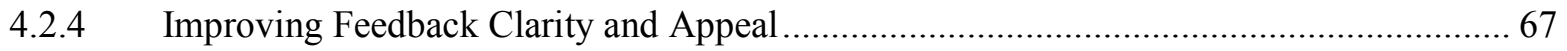

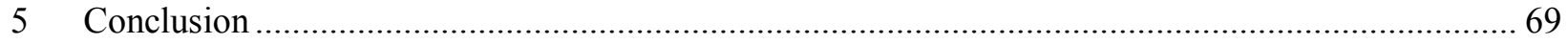

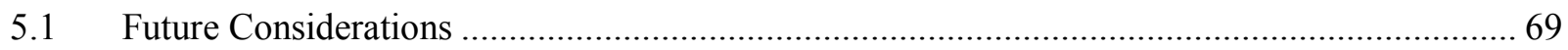

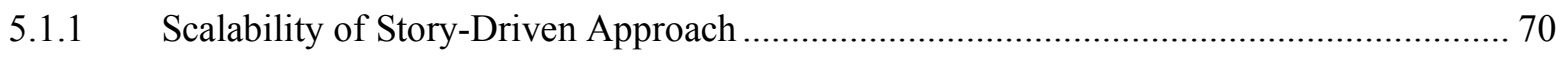

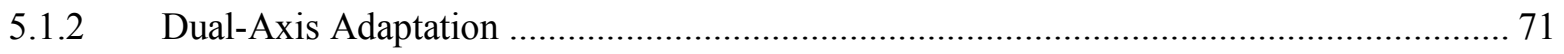

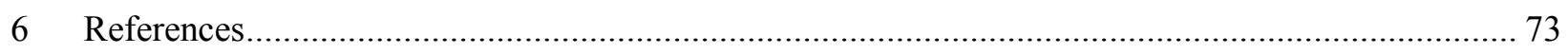

Appendix A Comparison of Formulas for Maximum Heart Rate …................................................ 79

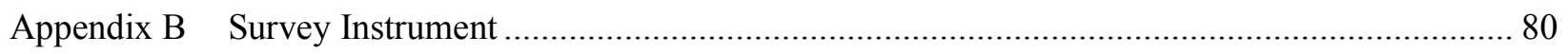

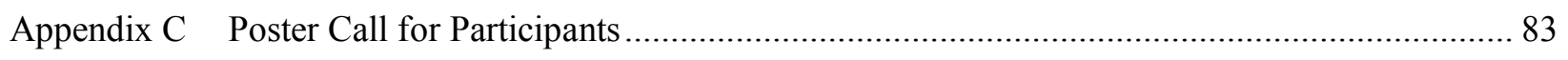

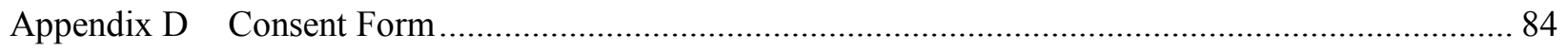

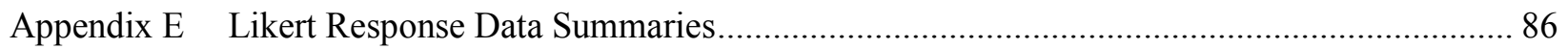




\section{Figures}

Figure 1 Peripherals worn by the user include the SNAP motion sensors (yellow) and the Zephyr HxM

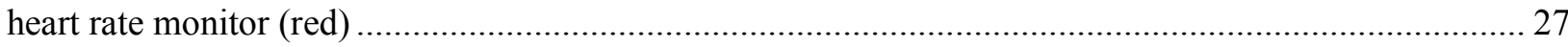

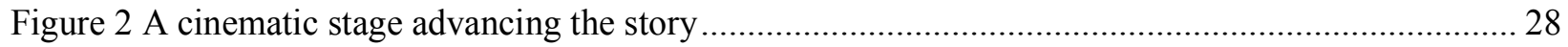

Figure 3 Action stages begin with animated notification of the expected exercise ...............................28

Figure 4 Players are represented by the middle character; the others provide an example to follow......... 29

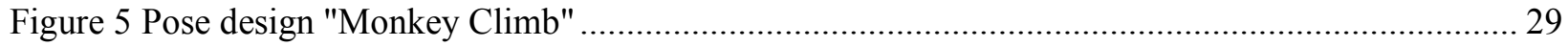

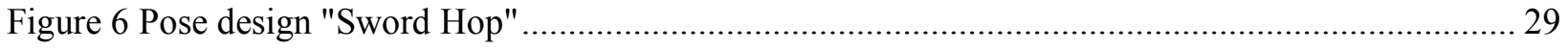

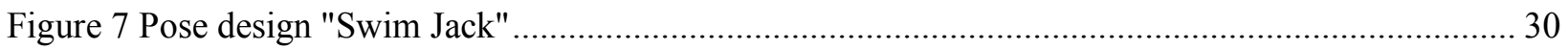

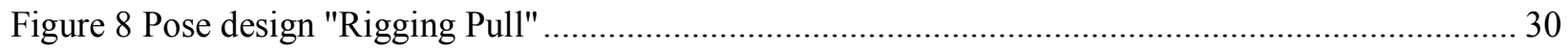

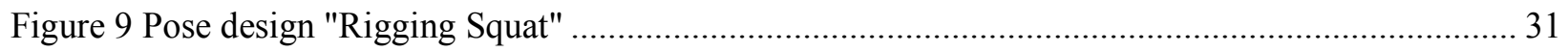

Figure 10 Hardware and software relationships supporting the game ................................................. 32

Figure 11 Simplified curves of intensity during the experiences of gaming and interval-based exercise. The similar curves indicate compatible designs, opening the door for exergames to combine them into one

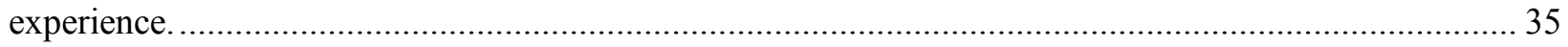

Figure 12 The adjustment curve used by algorithm in the case where the player is below HRtarget. The progress axis represents the player's current position in the range from HRrest to HRtarget.

Figure 13 The adjustment curve used by algorithm in the case where the player is above HRtarget. The progress axis represents the player's current position in the range from HRtarget to HRmax.

Figure 14 Average proportion of time spent in heart rate reserve intensity zones in each action stage, for all players overall, for both rounds averaged.

Figure 15 Typical HR result from a single participant illustrating interval training implementation 


\section{Tables}

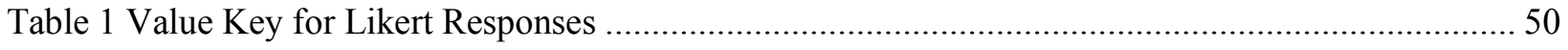

Table 2 Variance of Time Participants Spent in Exercise Ranges ..................................................... 56

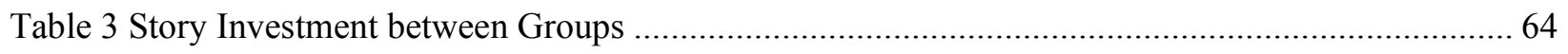




\section{Equations}

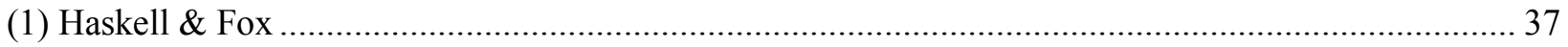

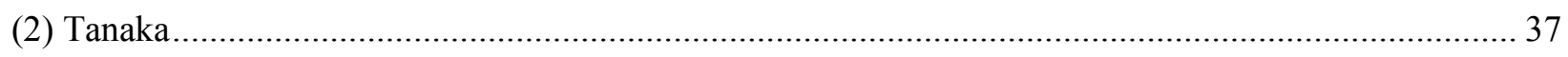

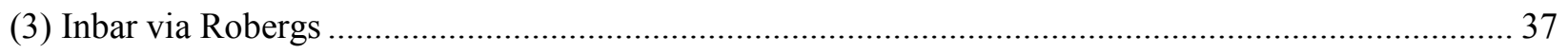

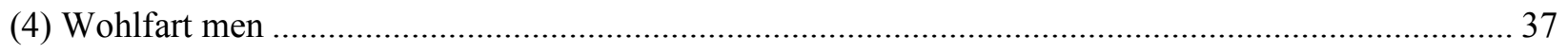

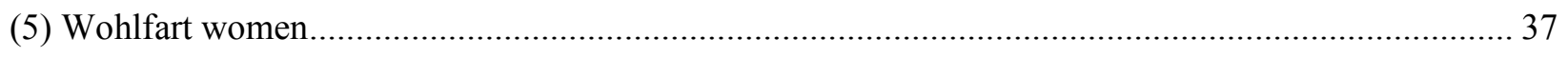

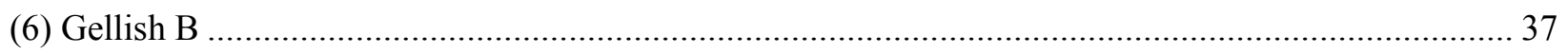

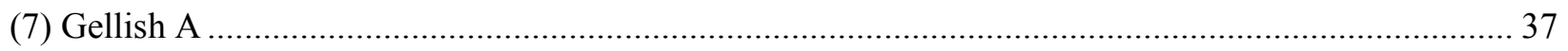

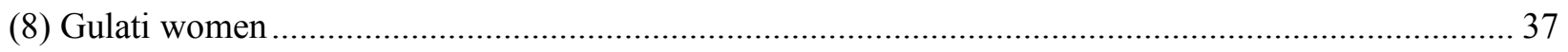




\section{Introduction}

In the video games industry, novel control mechanisms are continuously explored. Most games use a standardized control mechanism found on major gaming platforms, such as keyboard and mouse, or home console button pads. However, the history of the medium has seen many attempts at alternatives involving other devices. In recent years, there has been a serious resurgence in alternative control devices, with all major home consoles competing for sales in motion gaming technology, including Nintendo's Wii [39], Microsoft's Kinect [13], and Sony's Playstation Move [72]. Mainstream attention and widespread consumer adoption of motion control technologies has opened the door to a secondary trend towards exercise gaming, or "exergames". These are video games which promote physical motions that ostensibly result in beneficial physical exercise.

The term "exergame" has been employed to describe a game whose gameplay can result in beneficial exercise, regardless of whether that is the core intent of the game's design. One of the most effective and efficient exergame designs remains Konami's Dance Dance Revolution [55], using only a dancepad to control a rhythm-based motion game. Yet, $D D R$ 's design is presented simply as a simulated dance experience, focusing on entertainment value, without explicit regard to exercise benefit. More recently, games such as Nintendo's Wii Fit [39] and Ubisoft's Your Shape [78] series have put more explicit focus on the notion of gaming as exercise.

\subsection{The Nature of Exercise Games}

Games can be a powerful element of our lives. Many development initiatives, as well as a vast commercial industry, revolve around the human fascination with games. Because they are very sophisticated systems, studies in many disciplines have contributed to their advancement and reacted to their success. Because they are so powerful, games have been leveraged to address issues other than having fun, creating interesting interactions or conveying a story. One such issue is exercise and physical fitness. It is not disputed that there are substantial benefits to regularly getting physical exercise. Exercising is commonly known to be conducive to weight management, to reduce the risk of disease, and to promote overall better physical condition. 
Exergames, games that incorporate exercise, have cohered as a genre in the eyes of researchers and consumers. From purely entertainment-based beginnings such as Dance Dance Revolution, exerciseenabling games such as the Wii Fit have carved out a significant market by appealing specifically to the desire for physical fitness. Studies into exergames are generally motivated by a health crisis in the United States and elsewhere, and seek to leverage and expand the appeal of physically beneficial games. The general outlook for exergames is that they have potential as an alternative fitness solution for people who are not motivated to exercise by conventional means.

The outlook for exergames as contributors to fitness is very promising, but the road ahead is not entirely clear. It has been shown that the enjoyment of gaming is sufficient to motivate exercise in the first place [18]. Exergames have been integrated into successful community fitness programs [10], enhancing the programs even without assigning specific gaming goals to participants [61].

\subsection{Why Study Exercise Games}

This study examines exergame design from the perspective of the dual-flow model [45], which suggests two major components of success. These two components imply research goals for exergame design:

a) Maximize the effectiveness of exergames, delivering an exercise regimen to increase and maintain physical fitness.

b) Expand the appeal of exergames by optimizing attractiveness to players, thus maximizing the number of people who will play and reap the health benefits.

The first goal, maximizing efficacy, refers to whether the player actually receives a workout that is beneficial. A distinction can be made between a game enhanced by physical interaction, and a beneficial exercise session enhanced by integrating a game experience. The Nintendo Wii is a leading example of the former. Densely populated with applications, it is a system that largely uses physical interactions for the sake of novelty, creatively enhancing the possibilities of gaming interaction, without necessarily offering meaningful exercise. However, we will focus on the latter group: exergame design as an endeavor to support the exercise habits and physical fitness of users by offering a gaming experience that can make this task enjoyable and self-motivating. Towards this focus, the notion of "gamification" [22] will be examined. Gamification is the process of enhancing a non-game experience using game elements. By understanding robust gamification, we can relate it to exergames as a design process. 
The second goal, expanding appeal through optimized attractiveness, refers to how enjoyable the game is according to a wide audience of players. An important aspect of enjoying a game experience is that the level of challenge encountered by the player is appropriate, neither too difficult nor too easy. This goal creates a bridge to the realm of player modelling, i.e. the creation of data models that can represent the player, her actions, her needs and preferences, and more. Player modeling is the key to a variety of techniques for creating adaptive games, imbuing games with functionality for making changes to better suit players, also known as adaptivity. The ability to make changes after development time and during playtime has the potential to radically increase the appeal of the game to players in several ways: by discerning universal values, by indentifying values of a subgroup, and even by modeling the values of individuals. By creating one product that can achieve appeal in this dynamic way, more players can enjoy the game more thoroughly. This can enable the maximum audience to receive health benefits, fulfilling the second goal of exergame research. Furthermore, because exergames have multiple initiatives, integrating adaptivity could support them in multiple ways. Adaptivity can enable adjustments that are conducive to both of the above goals, effectiveness and attractiveness.

The needs of exergame design have been unfulfilled in many cases. Although they have taken many interesting forms, many on the market are not yet living up to their potential. Examining Wii Sports, one of the most popular physical interaction video games, Graves [19] said that the energy used when playing Wii Sports was not enough to contribute towards the recommended daily amount of exercise in children. Regarding mobile device-based exercise apps, Pagoto [38] concluded that mobile apps are underperforming and do not include good features to promote prolonged weight management. Even in academic studies, creating a physically beneficial gaming experience that will last over the long term remains challenging, such as in Lin's [32] study where most players' enthusiasm in the game decreased after the first two weeks.

Design strategies to fulfill the two goals of exergames are still not well understood. For long-term success, the design of exergames requires the consideration of both effectiveness and attractiveness. We present a study employing a story-driven exergame, with a full-body pose-based control device, in order to examine a method for addressing both effectiveness and attractiveness issues. As will be discussed, synthesizing these considerations requires a robust gamification, supporting an appropriate fitness routine with fundamentally integrated game mechanics which result in the same level of attractiveness as conventional entertainment-based games. 


\section{$1.3 \quad$ Thesis Overview}

After research questions and contributions are established, the theoretical background of our study is explored. Rather than focusing on exergames at the hardware level, the exploration is initially of an experiential theory which is applicable to both physical exercise and gaming: psychological flow. We review flow by dividing its applications into categories to gain perspective on its wide applicability, eventually narrowing down to gaming. Then, we turn to a design approach that can unite exercise with gaming: gamification.

We proceed to examine the dual-flow model, an application of flow theory for exergaming that can help us to apply gamification. The framework introduces a need for adaptivity, the capacity for the game to make adjustments to itself at runtime in response to players. Adaptivity for gaming in general is reviewed, as well as its applications in exergaming studies.

The methods for this study begin with a game design overview, introducing the broad concept and major features of our original game, created to investigate our research questions. This includes defining our objectives for invoking physical exercise in players, and how we measure it. We explain specifically how we apply the frameworks and guidance we have reviewed in our theoretical background, and how we evaluate our success in that application.

Results are presented by interpreting data collected from play sessions with the game, and player responses to our survey. Responses to our research questions, as well as our apparent levels of success in applying our theoretical background, are expounded. Implications and limitations are discussed. Finally, we draw conclusions for the study, and outline considerations for future studies in our lab, in labs using similar equipment, and in exergaming research in general.

\section{$1.4 \quad$ Research Questions}

Two main research questions are posed. The first employs a study with two groups to examine the effect of the presence or absence of adaptivity. The second gathers perceptions from players in general, but does not rule out effects of adaptivity. 


\subsubsection{Adaptive Exergaming}

For this study, we treat exergames as an approach to fulfilling the dual goals of attractiveness and effectiveness. These goals promote a state of psychological flow, which has multiple results. A state of optimal performance yields autotelic enjoyment, which serves the exergame's value as an entertainment source. In the context of an activity which is equivalent to physical training, it also yields a productive performance that can maintain or improve the player's level of fitness. This serves the exergame's core value of fitness benefit, which can apply to more specific benefits such as weight management.

Reaching the dual goals requires that players experience an appropriate level of challenge, in both a gaming sense and in a physical sense. The exergame must be neither too hard, nor too easy. In conventional game design, creating an appropriate level of challenge is a matter of iterative fine-tuning at design time. For an exergame, however, challenge depends strongly on the individual player's levels of strength, speed, and coordination. These levels vary widely between individuals. Although a person's level of fitness can be improved over time, in an exergaming context, that immutable variation presents a hard problem. There is no 'ramping up' to be done in the short term; the player cannot just try harder to overcome a level of challenge which is poorly matched to their physical condition. What follows are frustration and fatigue (if the challenge is excessive) or boredom and possible degeneration of fitness (if the challenge is lacking).

This study investigates an avenue to overcome this hard problem: adaptivity. Specifically, an individual data model of the player supports fine-tuning of gameplay at runtime. Adaptivity is applicable to a broad range of games [70], including exergames [2,49]. In this case, the application of the adaptivity is challenge management - adjusting the level of challenge encountered by the player in order to promote the kind of balanced experience described by the dual-flow model, in terms of attractiveness and effectiveness.

Research Question \#1: Does individual adaptivity for challenge management improve exergaming experience? 


\subsubsection{Story-Driven Exercise Engagement}

Our approach to exergame design incorporates the idea of "hiding the exercise in plain sight" as discussed in section 3.5.2. That is, the nature of the game as an exercise aid, where a major component of the interaction involves physical exertion, is not averted from the player's attention. Rather, the player maintains a degree of awareness of their workout performance in an experience that contextualizes that workout in the world of the game, integrating it with the story.

The intended result of this approach is that, instead of forgetting all about exercise, the player discovers that it can be enjoyable when presented in this integrated way. The study serves as an initial test of the validity of this approach, which we believe represents a robust gamification of exercise. We conduct direct questioning about enjoyment and emotional investment. As well, an established survey tool for measuring flow is employed to detect experiences of flow and autotelic enjoyment.

Research Question \#2: How enjoyable is the game with regards to its format as a workout experience integrated as a story-driven game?

\section{$1.5 \quad$ Study Overview}

Towards addressing the research questions, a study was conducted with player $\mathrm{s}(\mathrm{n}=25)$ playing an original exergame and completing a survey afterwards. Towards Research Question \#1, the study has an independent measures (i.e. between-subjects) component where the players are divided into two groups. The experimental group plays with adaptivity turned on, while the control group plays without adaptivity, at a default challenge level. Main measurements include the proportion of time the player spent in the targeted zone of exercise intensity.

Research Question \#2 is investigated primarily using results from the whole pool of participants, although effects of adaptivity between the two groups are not ruled out while considering related issues. Main measurements include responses indicating enjoyment and investment in the story.

In the case of both groups, a study session involves resting to attain a baseline heart rate measurement, equipping the game input sensors, and training into the pose databank to prepare for play. Then, gameplay occurs for a total of about 20 minutes plus a break. Finally, the survey is taken. Full details on the study are explained in section 3 . 


\subsection{Contributions}

This study joins a growing body of work in adaptive exergames [2,34,49], and is among the first to do so using a full-body interface [37]. Viability is supported for exergames using an interval training style of exercise, as opposed to continuous motions through play. Introducing a story into an exergame using this interval training style is demonstrated.

The work implements several existing frameworks, supporting them as theoretical backdrop for exergame development. Integration of exercise into a gaming experience is approached from gamification perspective [22]. The dual-flow model [45] informs our adaptivity design, which we position using Smith's taxonomy [47]. Inspired by the GameFlow framework's [53] integration of psychological flow into game studies, we evaluate player experiences from a perspective of flow using the structured questioning approach provided by the Flow State Scale [26].

Biometric measurements suggest that our adaptivity offers increased reliability in the level of exergaming challenge encountered by the player. Those measurements are in the form of heart monitor data. We find it worthwhile to continue investigating adaptivity as an element of game development that can serve particularly sophisticated contexts, such as exergaming, which must combine an effective workout with an attractive game.

Survey results show that exergaming contextualized within an expandable story which unfolds over the course of the game was compelling and did not detract from the experience or the workout. This is affirmed by indications of flow experience and direct responses from players. That affirmation stands to incrementally broaden the conceptual realm of how an exergame can be designed.

As an auxiliary result, some differences between participant groups were observed that are attributed to the relative degree of prior motion gaming experience of participants, based on a gender disparity. Implications are outlined for supporting inexperienced players, and leveraging experience, in order to maximize content value for game creators, as well as the exergaming benefit for players. 


\section{Background}

\section{$2.1 \quad$ Exertion and Engagement}

Research has begun on design approaches and game elements for exergames. Yim [71] reviewed several types of exergames to analyse design elements that promote player motivation. Several requirements for motivating players were outlined:

- Integrate music. Music can generally enhance engagement and has been used as integral to gameplay in exergames such as Dance Dance Revolution.

- Facilitate leadership for novice players. In games with unconventional form factors and methods of interaction, such as exergames, the learning state is paramount, and guidance can assist greatly with player integration.

- Provide achievable short-term goals. Players can more easily summon immediate motivation to reach manageably visible and reachable objectives.

- Hide player's fitness level. Depersonalizing the game context can let players forget about their own identities while engaging the game world. This can avert personal insecurities about fitness and physical exercise.

- Avoid systematic barriers to grouping. Also, actively assist players in forming groups. Social or multiplayer exergames should be designed for a streamlined interpersonal experience. This ensures maximum leverage of competition-based motivation.

More generally, a successful exergame design needs to account for the two primary goals described in section 1.2: Effective physical exertion, and sustained player attraction [45]. For a robust design, both of these goals should be supported by a unified consideration of what is known in psychology as 'optimal experience', or flow. 


\subsubsection{The Concept of Flow}

The concept of 'flow' stems from Csikszentmihalyi's research on 'optimal experience' [12]. These terms refer to a state where a person performing a task experiences a merging of action and awareness, where external challenges and the internal skills to face them are balanced, and both are at a relatively high level. It is the state where a person performing exerts an above-average level of skill and efficacy to solve problems, and perceives an above-average sense of enjoyment by doing so. Csikszentmihalyi's original definition of flow, hereafter referred to as 'classic' flow, is comprised of nine components. The short codes representing each component come from [57].

1. Challenge-Skill Balance (CHAL): In flow, there is a feeling of balance between the demands of the situation and personal skills.

2. Action-Awareness Merging (ACT): Involvement is so deep that there is a feeling of automaticity about one's actions.

3. Clear Goals (GOAL): A feeling of certainty about what one is going to do.

4. Unambiguous Feedback (FDBK): Immediate and clear feedback is received, confirming feelings that everything is going according to plan.

5. Concentration on Task at Hand (CONC): A feeling of being really focused.

6. Sense of Control (CONT): The feeling of being able to deal with the situation and exert some amount of power.

7. Loss of Self-Consciousness (LOSS): Concern for the self disappears as the person becomes one with the activity.

8. Transformation of Time (TRAN): Time can be seen as passing more quickly, more slowly, or there may be a complete lack of awareness of the passing of time.

9. Autotelic Experience (ENJY): A feeling of doing something for its own sake, with no expectation of future reward or benefit. This is also described as intrinsic enjoyment. 
The flow concept and its components have been applied to analysis of human experiences in a wide variety of fields. Many of these studies have been toward a more explicit understanding of the flow state and its elements in certain contexts $[8,11,46]$. Others have approached flow as an analytical tool towards optimum performance of a task [30], or improving design decisions [53,68]. Still others employ flow within, or parallel to, another framework to create new perspective on difficult problems [9]. Gaming studies have readily accepted the flow concept because its components are readily relatable to concepts in gaming literature [53], identifiable in a gaming experience, and familiar to many gamers.

The next section provides an overview of flow literature in games as well as other disciplines. The following section extracts lessons from the literature review which pertain to studies involving flow. The degree to which we apply those lessons in the present study will be explained in the Methods section.

\subsubsection{A Review of Flow}

This section reviews literature from several disciplines that has, over the past two decades, created, examined, or incorporated frameworks for flow. The review includes such a range of different studies on flow in order to reveal the breadth and variety with which the flow concept has been applied.

McLellan proposed that the primary unit of transaction in contemporary society is experiences, as opposed to products or services [36]. Her driving point is, stated simply, is that everything is becoming an experience, and all experiences are designed. The argument for universality of experience design supports our wide-reaching review of flow and the domains it has touched. By cross-examining various approaches we can gain insight into what makes them effective overall.

Flow studies have looked at various audiences including athletes [57], online game players [9], Website users [46], knowledge workers [41], college students [43] and children [23]. They have treated flow in several ways, including incorporating the concept into design considerations [14,63], evaluating designs after the fact [53], and optimizing task performance [30]. 


\subsubsection{From Csikszentmihalyi's Works to User Experience and Web}

Csikszentmihalyi and LeFevre [11] examined flow in the workplace and elsewhere using random sampling, putting focus on the balance of skills and challenge while looking for emotional responses. The result was somewhat paradoxical, indicating that people experience significantly more flow at work than in leisure, despite the intuitive preference for leisure. This may mean that people have difficulty selfexamining their preferred activities because of socialized notions, e.g. work is not as enjoyable as time away from work. However, although the sample of people was highly varied, it seems (as the author cautioned) difficult to draw clear-cut general conclusions from a framework that operates only on the two axes of challenge and skill.

Ghani and Deshpande [16] looked at flow in human-computer interaction to examine the relationship between flow and task attributes like scope and autonomy. They found that task scope was a primarily associated with flow, meaning that the scope of tasks in new software should be a consideration in preparing the software for acceptance by an audience. This study casts a very wide net over a highly varied domain, making it appropriate for later studies to investigate flow in smaller HCI domains with more focus.

Chen, Wigand and Nilan [8] focused more specifically on web usage, conceptualizing the web as a "multi-activity medium" to account for different possible task scopes. They also conceptualized flow itself not merely as a holistic combination of the nine classic components, but rather as a structural framework with antecedent conditions, experience elements, and post-experience effects. The results found significant flow association with "searching" or information-seeking web activities. Skadberg and Kimmel [46] also found flow experiences in online searching when they focused on an even narrower case study of an informational website. They found flow to be most correlated with the speed and attractiveness of the site, which enhanced interactivity, surpassing the significance even of user skill (vital to the classic CHAL component). It seems that some primarily absorptive activities like web searching are sufficiently interactive to create flow experience, but the weighting of contributing factors is different. 


\subsubsection{The Flow State Scales and Behavioural Viewpoints}

Tenenbaum and Fogarty [57] evaluated Jackson's [57] Flow State Scale (FSS), a popular survey instrument for evaluating flow experience. The FSS and its successor the FSS-2 [25] have been used primarily in athletics, but the FSS has been adapted to other realms such as cognitive evaluation [40]. It is a relatively context-independent measure of the degree to which a subject has experienced flow in an activity. Tenenbaum evaluated the FSS, finding no significant reasons to alter it [57]. However, even with such a rigorous affirmation, it seems the validity of an FSS-based data pool should not be written off as a non-concern, because a survey instrument is a self-reporting mechanism (rather than an objective measuring mechanism) that can be deployed in a variety of ways. Ghani [16] noted matching concerns with reliance on self-reporting, and Rogatko [43] discussed its drawbacks.

Studies have employed flow to examine online games from the perspective of behavioral analysis, contributing to the understanding of addiction [9] and technology acceptance modeling [21]. Hsu's [21] framework integrates flow elements to gain new perspective, but the survey employed seems problematic: the concept of flow is introduced directly to the participant. Flow seems like an overly academic concept to put forth to a user who is expected to reflect and respond naturally on recalled personal experiences. Hsu's results appear consistent with Chen's [8], but this does not alleviate the issue. In fact, Chen notes difficulties for participants in understanding terms from the flow literature like "challenge". Rogatko [43] confirmed that the phrase "in the zone" is a suitable layman's term to express the concept of flow. Layman's terms like this should be used when writing for survey participants who may not (and do not need to) completely understand the academic concept of flow. Chou's survey accords with this guideline, and was reused later with success by Gackenbach [14]. Similarly, Inal and Cagiltay [23] made sure to remove items from their scale that their participants (school-age children) would have been unlikely to understand. 


\subsubsection{Distinguishing Conditions from Perceptions}

Quinn [41] employed a structural flow framework similar to Chen's [8] while examining flow in knowledge work. Several data collection methods were used, including interviews and a focus group, which seems to make this study more robust than just a survey. The study was successful but the structural outlook on the flow concept seems to be incompatible with the holistic outlook employed in Jackson's FSS [26]. Quinn concluded that people experience flow differently depending on job and task type, whereas the holistic model seems to imply that flow is the same in spite of the activity, and its degree depends mainly on the inclinations and skills of the individual. The two outlooks were compared by Beard and Hoy [4] in a study that evaluated their relative applicability and quality in the context of elementary school teachers. There, the Quinn flow model (used with knowledge workers) was expected to fit better with teachers than would the Jackson model (used most often with athletes). However, the result was, unexpectedly, the opposite.

It seems that the relative quality of the two scales may not be the best clue to understanding this result, but rather the subtle difference in conceptualization may be the key. The Quinn model dictates antecedents, conditions about a flow situation that are established before any experience takes place, such as the presence of challenges that will be balanced against the skills of the subject (CHAL). However, classic CHAL has also been described as the perception or feeling of balance between challenges and skills (by Csikszentmihalyi as repeated by Tenenbaum [57]). A feeling can definitely only occur during an experience, rather than before it. The Jackson model subscribes to this view, evaluating all components at once as part of a unified flow concept, during the experience. This conceptual difference between the two frameworks, and its implications on studies comparing them, is worth investigating in the future. For instance, Payne et al. [40] performed a study where this distinction may have been useful; the study uses an adapted version of the FSS (the Jackson model) but the analysis of the results seems to subscribe to the antecedent-based structural viewpoint of flow (the Quinn model), seeking to frame the circumstantial balance of challenge and skills in advance of any experience. 


\subsubsection{GameFlow's Issues and Success}

Sweetser and Wyeth [53] present GameFlow, a framework that stems from the classic nine-component flow concept, overlaying it with concepts from game design studies. The result is a framework for evaluating games that includes most of the classic components and adds a social component. The social component is interesting but it seems to be at odds with the individual spirit of some of the components in classic flow, especially LOSS (the diminishing sense of self). For instance, a participant in an FSS survey might indicate that they were "not worried about others" [57] during their experience. Inal and Cagiltay [23] use an approach that can contend with this issue, analyzing social interactions and gameplay interactions as two separate forces that can mutually but distinctly influence flow experience, instead of merging the two interactions into one framework. Another issue with GameFlow is that the ACT component of flow (the automaticity of action) is undervalued. ACT is one of the most distinctive and potent components of flow experience. It should be more directly incorporated with GameFlow's "Player Skills" component as a measure of skills ramping up over time. However, the de-emphasis on ACT may be inherent in the nature of the GameFlow framework: it is a critical review tool. Users of the framework (ie. expert critics) must infer potential flow states in hypothetical users, rather than measuring them while evaluating designs. In this context, ACT may be very difficult to infer. Similarly, TRAN (the transformation of time) is minimally visible in GameFlow, and is lumped in with LOSS in its Immersion component. This deemphasizes a very distinctive flow component that is, in contrast, measured quite independently in the FSS. But again, this is due to GameFlow's purpose as a critical review tool, where TRAN is difficult to infer. Sweetser and Wyeth later refined a version of GameFlow to be more effectual in the specific context of real-time strategy games [52].

Despite the above issues, GameFlow has been widely cited and was later adapted by Jegers [27] to review pervasive gaming designs. Jegers adds a "pervasive dimension" to GameFlow components. The most interesting consideration to come out of this addition is the moderation of the Immersion component, since pervasive (mobile) players cannot afford to be totally immersed. This calls attention to a context where flow is desirable (ie. gaming) but difficult to achieve (ie. mobile situations). The Pervasive Game Flow framework does not offer explicit approaches to mitigate this difficulty, but it is still a good example of how a flow-based framework can be utilized and refined to explore the needs of a particular context. One can imagine a similar process to create versions of GameFlow to evaluate designs for other specialized gaming contexts, such as co-operative games, and games for audiences with special needs. 


\subsubsection{Performance, Timing and Preferences}

Lee and Kwon [30] examined flow with the goal of correlating it with game performance. They found that their subjects' achievement level was much more strongly related with the player's information processing strategies than with flow. However, their testing format had a limitation: the timeframe of user experience was only 30 minutes. Flow is a state of deep immersion where one may even forget one's own identity as separate from the activity (LOSS). In order for that to occur, a significant amount of time seems necessary to become immersed. Thames [58] explicitly recommended at least one hour for real flow experiences. Rogatko [43] also cautioned about timeframe, recommending a full hour instead of the 10 minute period used in another study. As a counter-example, Beume et al. [5] used 12-minute sessions, and LOSS did not even enter consideration in the results, suggesting that timeframe may have been a limitation there as well.

Inal and Cagiltay [23] examined flow in the gameplay and socializing experiences of school-age children over a long period of observation. They used a flow scale survey as a face-to-face interview, mindfully adapting to their audience. They found interesting gender disparity in what factors and conditions contribute to flow experience.

Beume [5] found curious results when examining the relationship between game difficulty and fun. The objective measure of difficulty was correlated strongly with perceived fun, but weakly with perceived difficulty. This seems like an indicator of the ACT component, where a player simply responds to challenges automatically without necessarily perceiving them as challenges at all. However, it could also be the result of an oversimplified model of flow. The study essentially focuses on the CHAL component (the balance of challenge and skill) but does not incorporate the aspect of both challenges and skills being at above-average levels (for each particular player) during the experience. This neglects the possibility of an "apathy" situation [11], where players are not sufficiently challenged and do not employ a sufficient level of skill to enter flow. This could be detrimental to a study focusing on fun and enjoyment.

Gackenbach [14] surveyed a variety of gamers and discerned that "hardcore" gamers have stronger preferences for certain kinds of games (according to structure or genre). The results indicate that a "moderate" level of "game dynamics" (the amount of features and activity in a game) is most conducive to flow. This seems to accord well with the classic CHAL component, since a "moderate" challenge would be perceived as "balanced". 
Wang and Chen [63] made a similar investigation that related challenge and preferences to learning performance in educational games. Their most notable finding seems to be a "compensation effect", where players who preferred non-challenging games but were matched with challenging games anyway actually learned more. However, it was also found that optimal flow did not actually result in optimal learning, posing a curious design consideration for learning games.

\subsubsection{Lessons in Flow Study}

Having reviewed and critiqued a variety of works across several disciplines, we can extract a number of lessons applicable to studies of flow.

Lesson 1: Allow sufficient time for flow experiences to occur while data gathering. One hour has been suggested $[43,58]$. Some studies $[5,30]$ may have been limited by insufficient timeframes for flow experiences.

Lesson 2: Use everyday language when asking survey participants about their potential flow experiences. Instead of introducing academic concepts, use layman's terms and try to make questions intuitive and natural, in order to facilitate natural answers based on intuition about one's experiences. The FSS [26] appears to be a good example of this. It has influenced the design of further studies $[23,43]$.

Lesson 3: Do not neglect ACT, LOSS and TRAN. They may be difficult to perceive and measure [57] and it may be tempting to aggregate them into a more easily applicable framework (see the critique of GameFlow) but these are some of the most distinctive components of the flow concept and may be important to distinguishing and qualifying the significance of a flow experience.

Lesson 4: If possible, mix self-reports with objective measures. Ghani and Deshpande [16] expressed concern about reliance on self-reporting, and Skadberg and Kimmel [46] explicitly called for more objective measures in future flow studies, suggesting cross-validation with different data in the meantime.

Lesson 5: If possible, use more than one framework. Beard and Hoy's [4] effort to compare two distinct frameworks yielded unexpected results that defied intuitive expectations regarding the frameworks' relative applicability to the domain at hand. Applying more than one conceptualization of flow in future studies may reveal implications about the relative domain suitability (or cross-domain compatibility) of each framework.

The way in which we apply these lessons is discussed in the Methods section. For now, our background review continues with a design approach for making exercise into a gaming experience. 


\section{$2.2 \quad$ Exergaming as Gamification}

This project examines exergame design as an endeavor to support the exercise habits and physical fitness of users by offering a gaming experience that can make this task enjoyable and self-motivating. Towards this goal, the notion of "gamification" will be examined. Gamification has generally been used to describe the process of enhancing a non-game experience (in this case, exercising) using game elements, but there may be significantly more to it than that, if the value of the user's experience is to be significantly augmented. By understanding gamification, we can integrate it as a design process for exergames.

\subsubsection{Exergame Benefits}

Staiano [50] provides a history of exergaming development and applications. This is organized mainly by type of device (eg. stationary bicycles, dance pads) and includes a brief critique of the strengths and weakness of each, relating these attributes to their history of success or failure. The review makes no distinctions between the core intents of exergaming products - games that were developed primarily for enjoyment (eg. Dance Dance Revolution) are held in equal regard with games that were developed primarily as a fitness incentive (eg. Wii Fit). What matters here is not the core design motivation, but rather the results.

Secondly, Staiano enumerates motivations for exergame study by examining various studies and products and citing positive effects observed in several categories. The first category is Physical Outcomes, divided into Caloric Expenditure and Heart Rate Increase (exergames have been seen to contribute to legitimate workout exertion as well as long-term fitness), and Coordination (exergames have contributed to skill transfer into real-world activities; while traditional games have been associated with improved hand eye coordination fine motor skills).

The second category is Psychosocial Outcomes, divided into Social Interaction (social exergaming has fostered human relationships, and there are cases where fitness effects have been sustained solely by interest in the assocated social interaction), Self-Esteem and Self-Efficacy (absorption in a game during exergaming may reduce self-consciousness barriers, and has been seen to increase self-esteen), Mood (exercise generally improves mood and exergames have been seen to be no exception), and Motivation (games are known to invoke motivation intrinsically, and this has been channeled to promote exercise with games as a reward). 
The third category is Cognitive and Academic Outcomes, divided into Attention (traditional games have improved task handling skills, and this may transfer to exergames), Visual-Spatial Skills (perceptual ability and speed have been associated with aspects of traditional games that may map effectively to exergames), and Academic Performance (games, including exergames, are related to problem solving and task-reward scenarios).

Finally, Staiano discusses the potential of exergames in school settings, in physical education courses. Advantages include competitive price, adaptability to space constraints, high allowance for sharing, and the potential to foster social interactions and self-esteem as a side-benefit.

Throughout the review, the authors make a strong case for exergames by taking advantage of both the exercise aspect (related to what we refer to as effectiveness) and the gaming aspect (related to what we refer to as attractiveness). Studies on general exercise benefits are associated with exergames; this seems justifiable, as the review also establishes that exergames have been seen to contribute to real fitness. Also, in the absence of research specifically on exergames, some categories cite traditional game studies, suggesting that the results may apply also to exergames. This seems like a reasonable assessment because the major appeal of exergames is to make exercising more game-like, or to integrate exercising into a game experience.

Gamification for physical fitness and weight management has already seen significant demand and success in the real world [3]. However, whether an exergame is just an attempt to add game-like elements to exercising or truly integrates exercising into a game experience is an important distinction.

"Gamification" has recently become the term to describe fusing non-game experiences with games. It has become something of a buzzword in some domains, and its validity as a methodology remains vague and questionable. Bogost [6] has severely critiqued its use as a buzzword from a marketing perspective. He indicates that gamification has largely generally produced shallow applications that do not create cohesive game experiences. Instead, they simply add elements that are perceived as characteristic of games, such as points, progress bars and trophies. For the gamification concept to yield real value and fulfill its potential, it is Bogost's position that the design process must be richer than just tacking on "incidental properties" of games. 


\subsubsection{Defining Gamification}

Huotari [22] builds on previous attempts to define 'gamification', offering a modern definition from a service marketing perspective. First, the argument is framed with a review of attempts to define games in general. There is emphasis on the difference between the two axes upon which the definitions can be situated: the systemic aspect of games, and the experiential aspect. Both aspects are shown to be identifiable in prior attempts in the literature to define gamification. Another important note is the lack of any literature identifying definitive aspects which are unique to games (ie. uncommon to other media or systems). This is explained as a symptom of the game's nature as the interaction of multiple conditions instead of a singular isolated aspect. It is proposed that existing definitions of gamification which only incorporates the systemic aspect of games (the rules, mechanics and outcomes) are inadequate in light of the experiential aspect (engagement, particularly enjoyment).

Secondly, Huotari provides an overview of major concepts from service marketing methodology. The concepts considered are 'customer as co-producer' and 'value-in-use'. Both essentially put forth that the service (or its value) is co-created partly by the service provider, and partly by the customer who interacts with it. The value of the service emerges only through the interaction of the customer with the designs implemented by the provider. This is the core of the experiential aspect that should emerge as a quality of a gamified system, in addition to the systemic conditions.

Thirdly, a distinction between 'core' services and 'enhancing' services is made. This is conceptually vital to the definition of gamification from a service-oriented perspective, because gamification is a process which acts in the role of an 'enhancing' service. This distinction is meant to clarify what exactly is being gamified. Next, these service concepts are applied to games, which are viewed as similar to services in that they are a process which provides value to customers (ie. players). This frames the importance of considering the experiential aspect of games and situates the proposed definition of gamification: "a process of enhancing a service with affordances for gameful experiences in order to support the user's overall value creation".

This new definition is presented in contrast with a competing one which is based purely from a systemic perspective. The competition definition of gamification does not incorporate the experiential aspect, at least not to the fundamental degree that the new one does thanks to insight from service marketing methodology. 
Exergames, games meant to encourage exercise, seem to be an application of gamification in the sense of this new definition. In the context of exergames, the 'core' service is a fitness regime, with the goal of enhancing the user's fitness level through exercise. The 'enhancing' service is the mediation of the player's interaction with the regime through gameplay, with the goal of disguising the regime as a game instead of an exercise routine. (The degree to which it is disguised may vary, but as long as there is sufficient enjoyment leading to productivity, it may not matter.) The gamified service is the motivation to exercise, gained by interacting indirectly with the fitness regime through an intrinsically interesting game.

The service marketing perspective can be extended from the process of gamification to the creation of games themselves. Games can be viewed as more than the aggregation of systemic conditions. An experiential aspect can be added to the philosophy of their creation and validation. Since games are ultimately exercised and manifested through play, we can apply the 'co-producer' concept to consider the player as an integral creator of the game. This would accord with Adams' framework for considering games, particularly exergames, as a structure of contingencies [1]. Such contingencies are composed of antecedents, actions, and consequences, vitally involving the player in the creation of emotional game experiences.

We have examined a perspective of gamification, a process with multiple initiatives and specific priorities, which seems compatible with the multiple initiatives of health-focused exergaming. Next, we will examine a theoretical model for evaluating exergame design, in order to see how these multiple initiatives can be expressed in a more explicit way.

\subsection{Designing Exergames}

Sinclair's [45] 'dual-flow model' focuses on defining and supporting the initiatives of gaming enjoyment and exercise efficacy in parallel. It frames a theoretical model that encompasses and organizes vital considerations for the design of fun and effective exergames. The authors propose to approach exergame design using a dualistic model with two major components: factors of the effectiveness of exercise games with regards to providing actual exercise, and factors of attractive experiences that provide experiential value to the user and support the effectiveness of the exercise by encouraging frequent play. They construct both components in the fashion of Csikszentmihalyi's classic 'flow' construct. 
The dual-flow model is supported by studies asserting that the exertion invoked by exergames occurs on a scale relevant to the player's overall health and fitness [33]. These studies also establish the appeal of games in relation to exercise, finding that players are willing to engage in strenuous exercise even in advance of any entertainment presented by games or other media, where the entertainment is presented later as a reward [44]. Sinclair's review covers findings that introduce the importance of adaptivity, which can dynamically sustain the appeal of the experience, improving attractiveness and thus effectiveness overall. In particular, Yannakakis et al. [69] concluded that although the balance of challenge and skill is universally important for enjoyment, the actual quantities of those two factors are dependent on individuals.

The two components of the dual-flow model are built by incorporating insights from the review of studies to construct two parallel submodels of flow: 'attractiveness' and 'effectiveness'. Attractiveness is closely related to the classic measurement of flow, seeking to balance game challenges against player skills. That means avoiding both frustration (excessive challenge) and boredom (insufficient challenge).

Attractiveness is based on Csikszentmihalyi's original elements, and GameFlow [53] elements are also considerations. Effectiveness describes the balance between workout intensity and player fitness, sustaining and escalating both elements at a productive rate. Effectiveness means avoiding both failure (excessive intensity) and deterioration (insufficient intensity).

Finally, the authors present the need for adaptivity towards fulfilling the requirements presented by the dual-flow model. They suggest two methods. The first is game designs that focus intensely on the input, such as Dance Dance Revolution, which makes great allowances for creating game levels with different levels of difficulty, enabling designers to include content that is appropriate for a wide variety of skill and fitness levels. The second method is 'feedback'-based adaptation of game difficulty that takes each player's skill level into account. Yannakakis' "Bug Smasher" project is cited as exemplary for this method. To introduce the importance of adaptivity, the authors cite Yannakakis to demonstrate the applicability of dynamic game adaptivity to exergames. Yannakakis is a leader in the field of game adaptivity and procedural content generation. The works of he and his colleagues recently culminated in a framework called Experience Driven Procedural Content Generation (EDPCG) [70]. The framework proposes a set of design methods and implementation techniques that allow games to become "experience-driven", ie. primarily adaptive to the experiences, and the resulting feedback, of players. In the case of the dual-flow model, the kind of system suggested might be defined in the terms of Yannakakis' EDPCG framework as a data-driven system, with directly represented content and gameplay-based experience monitoring, and using a data model against which fitness data can be compared. 
We can consider the dual-flow model from the perspective of the service marketing definition of gamification, and it seems to accord very well. Both of these perspectives examine systems with multiple, sometimes contradictory, goals. They both examine an experiential aspect - for gamification, this is value-in-use, and for dual-flow, it is game attractiveness. The experiential aspect is examined in parallel with, and with at least as much importance as, a systemic aspect - for gamification, this is the core service, and for dual-flow, it is exercise effectiveness). These similarities suggest that the service marketing perspective definition of gamification is compatible with the dual-flow model for evaluating exergame design. Their compatibility makes it even more compelling to see exergame design as an application of gamification.

\subsection{Exergaming Incorporating Adaptivity}

In exergame design, a major goal is to expand the appeal of the game in order to maximize the fitness benefit on as wide an audience as possible. Maximizing appeal can be assisted by employing techniques for optimized experience. This is a bridge from exergaming to the realm of game studies called 'player modelling', which has generated research on data models that can represent the player, her actions, her needs and preferences, and more.

Player modeling is the key to a variety of techniques for creating adaptive games, imbuing games with functionality for making changes to better suit players. The capacity to make changes after development time and during playtime has the potential to radically increase the appeal of the game to players by discerning universal values, indentifying values of a subgroup, or even modeling the values of individuals.

By creating one product that can achieve appeal in this dynamic way, more players can enjoy the game more thoroughly, enabling the maximum audience to receive health benefits. Furthermore, because exergames have multiple initiatives, integrating adaptivity can support them in multiple ways. As established in the dual-flow model, exergames need to be both attractive and effective, and adaptive games can implement adjustments that are conducive to both these needs. 


\subsubsection{Towards Adaptivity}

Smith [47] offers a taxonomy of player modeling as a tool to clarify this important method toward game adaptivity. "Player modeling" refers loosely to the design, population and analysis of a data model that is based on the player. It can support a variety of procedural techniques by which a game is modified after development time, in the time when the player is experiencing it, in order to optimize it toward some design goal. This description is broad and theoretical, and to narrow down what player modeling means in specific cases of practice is the intent of Smith's study.

The main points made initially are that player modeling has no single unambiguous meaning, the term is used throughout the literature in highly various ways, and even here the authors offer no unifying definition. Rather, the contribution is a survey of works and concepts, which is constructed as a taxonomy of various kinds of player modeling. For any given work from the nebulous world of player modeling, qualifiers may be chosen from this taxonomy to describe more precisely the scope, intent, mechanisms, and results of a given system.

The taxonomy is divided into four facets that together describe a player modeling system: The domain from which data is extracted, the purpose of the model in terms of changes made to the game, the scope of the player model in terms of what kind of population it represents, and the source of insight or method used to interpret the player model to make decisions. Each facet has several defined values which are described and exemplified by applicable works.

This contribution of this framework is two-fold. For one, it has the progressive goal of not carving out a definition, but instead introducing qualifiers to refine and already ambiguous term. By taking an inclusive approach instead of setting out to establish an exclusive definition of the term, this study creates a widely appreciable framework. The contribution is also supported by the study's non-dependence on existing examples. It does more than just categorize a set of works; it frames an encompassing range of concepts that also represents hypothetical and theoretically logical systems.

In conventional video games, adaptivity can support a variety of design goals, such as challenge, variety, and preferences. In the case of exergaming, we have two specific design goals which are important, attractiveness and effectiveness. It remains to examine how designers could actually approach using adaptivity to support exergaming goals. For this insight, we will examine Gobel's [17] framework for adaptive exergames, which combines several components in a structure designed to enable adaptive exergaming experiences. 


\subsubsection{A Framework for Adaptive Exergames}

Gobel [17] examines exergames from the perspective of 'serious games', a category for games that are primarily motivated by significant real-life purposes, such as education, training, rehabilitation, and (in the case of exergames) exercise. A framework is proposed that allows health professionals to author and modify exergame-based fitness programs. The framework includes an adaptive component, measuring vital parameters of the user in order to adjust gameplay. Data is collected from player behavior, as well as devices that observe the player's physical state, for the purpose of operating on a player model. The adaptive engine is itself adapted from a storytelling project, and different exercise sessions are organized as story segments. The most appropriate segment to visit next is chosen based on the type of player, according to collected data. In addition to this player-category-based decision making, the engine accounts for the important stages of a productive workout - warmup, the main session, and cooldown. Adjustments can also be made based on explicit user preference, i.e. asking the user what kind of intensity is desired.

Some existing work-in-progress exergames are also described. All of the games serve as candidates for applying the proposed framework to implement adaptivity, thereby augmenting enjoyability and optimizing workout efficacy. Ergoactive is a stationary bike platform with several applications based on speed and intensity as input, as well as auxiliary input such as hand-held motion controller. SunSportsGo is a biathlon-like game that combines running, input via leg-mounted sensors, and shooting, input via an additional sensor. It supports a simultaneous multiplayer mode. Y-Move is a head-tracking based game that uses bodily motion to control a car and navigate a racecourse. It also supports single and multi-player modes, opening the door to social engagement benefits described in GameFlow. 
The "story-based" approach to adaptivity suggests a particular kind of player modeling; it can be identified more precisely by using the taxonomy constructed by Smith [47]. Described with the terms of that taxonomy, Gobel's framework suggests a cross-domain application, with adaptivity being based on both in-game player actions, and the player's own human reactions. Its purpose is "descriptive", guiding a high-level course of action, as opposed to "generative", which would lay out fine details without relying on pre-existing modules of content. The scope is "class"-based, evaluating individual players as they fit into one of several parameterized groups. (These groups are defined by "annotating" exercise modules with player data values. The choice between modules constitutes the assignment of a player into a group.) The "source", or type of reasoning on the data used to make decisions (whether it is "induced", "interpreted", "analytic" or "synthetic"), is not specifically implied by the framework, but would instead by implied by the specific application. So, in Smith's terms, Gobel's framework represents an approach to action/reaction-based, class-based, descriptive adaptation in exergaming applications.

The next section commences the description of our game used in the present study. Gobel's study represents a valuable synthesis of several theoretical aspects - workout essentials, adaptivity, and gamebased flow theory. We have attempted a similar synthesis using a design inspired by other games in research such as DanceBeat [2] and Heart Burn [49]. We include a description of our game in terms of Smith's taxonomy in section 3.4.3 to position it relative to Gobel's study and other adaptivity research. 


\section{Methods}

In order to address our research questions, we present an original exergame which incorporates adaptivity for challenge management. Players experience a continuous story while interacting through action stages in which they must perform tasks contextualised by the story. The game is played with a pose-based motion sensor system, for which players wear a set of accelerometers. Players also wear a heart rate monitor so that the game can measure and record the degree of cardiovascular exercise being performed.

The participant game session is followed by a survey (Appendix B). The survey gathers insight into the player's preferences and gaming history, as well as their flow experiences during the session.

\subsection{Game Design Overview}

The game is original software which works with an existing hardware. Players interact by striving for combo scores in the context of a story which unfolds throughout play. It incorporates an interval training exercise design, and adaptation for challenge management of the workout.

\subsubsection{Input Platform: the SNAP System}

The platform of this study includes an gaming input system called the Sensor Network for Active Play, or SNAP $[64,65]$. It is composed of a networked group of acceleration sensors which are worn upon the body using straps, nearby highly mobile joints. In this study, the sensors are positioned at the wrists and knees as shown in Figure 1.

Collectively, the sensors are used to create a data model of a particular bodily pose. During an initial training phase, the user performs poses while a researcher uses administrative software to collect samples of sensor data to create the data model. Later, when the pose is performed again, the model may be used to determine if the user's current bodily pose approximately matches the pose they have trained into the system. The system can read when a user performs one of a set of poses during gameplay. As an exergaming platform, the SNAP system has been compared favorably against Wii Sports and others [66]. 


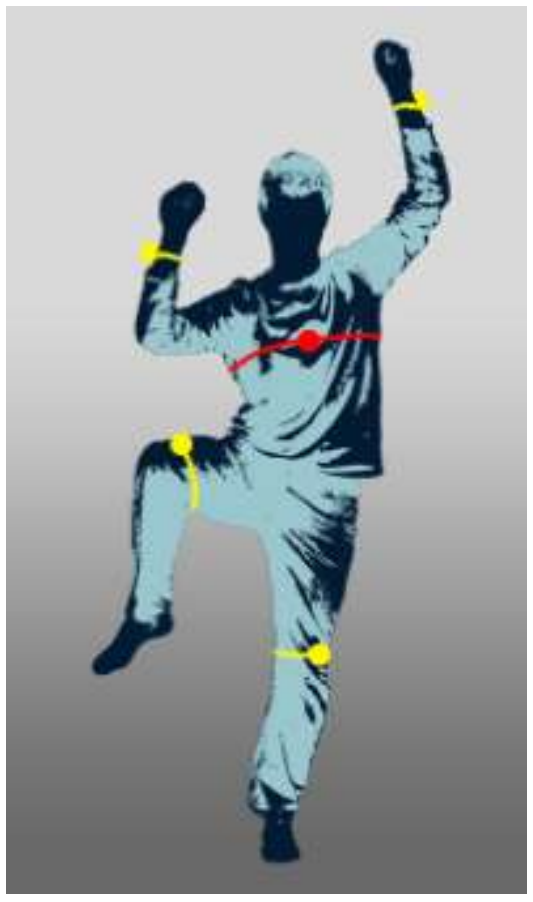

Figure 1 Peripherals worn by the user include the SNAP motion sensors (yellow) and the Zephyr HxM heart rate monitor (red)

\subsubsection{Software: Sea Monkeys}

Sea Monkeys is an original game designed for full-body pose control. It is meant to indirectly promote exercise through the gameplay of repeatedly stepping through workout-like poses. The game uses the SNAP system as its primary player input platform, in addition to administrative input from the researcher through keyboard. The underlying exercise design is described in section 3.3. The game can be set to adapt its level of challenge to suit individual players through adaptivity described in section 3.4. Gameplay is contextualised in a story-driven experience which is alternative to conventional exercise settings. The story-driven design is outlined in section 3.5. 


\subsubsection{Gameplay}

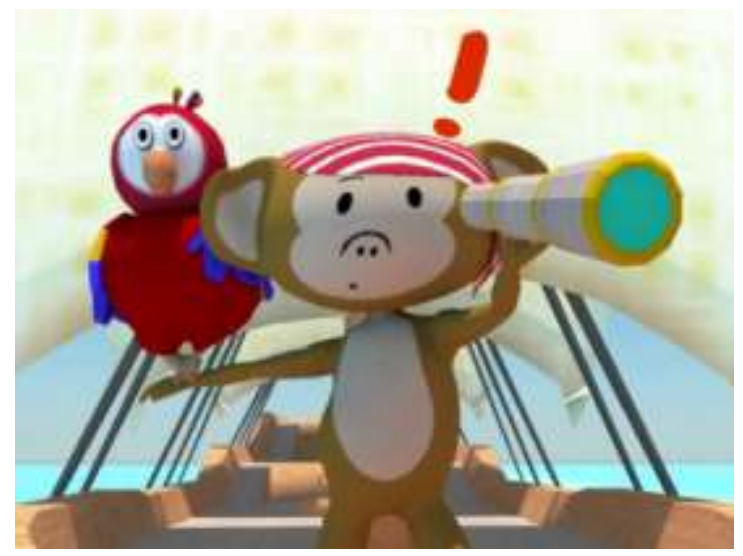

Figure 2 A cinematic stage advancing the story

At the outset of gameplay, the player views an introductory cinematic which begins the story. All cinematic stages are simply short movie segments (Figure 2). Their duration serves the player as a rest period between action stages. With the first cinematic finished playing, the game proceeds to an action stage, where the player is first reminded how to perform the expected exercise for this stage (Figure 3). Following a countdown, the player attempts to keep pace with animated characters that perform the exercise.

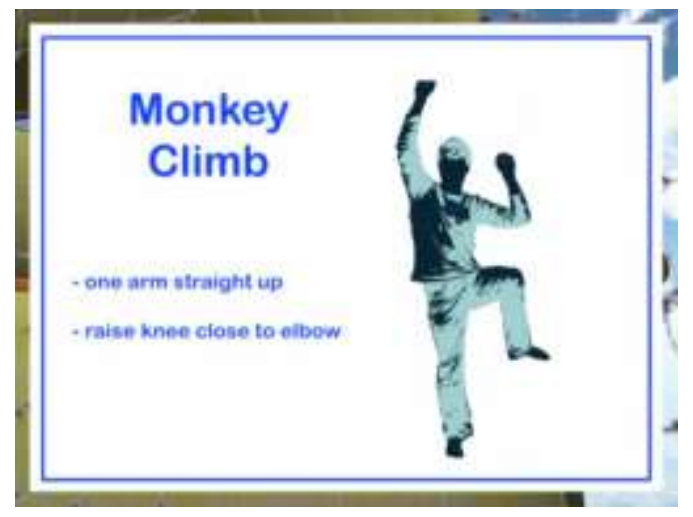

Figure 3 Action stages begin with animated notification of the expected exercise

As the characters animate, the player's task is to follow along, matching their poses. There are two types of characters for the player to watch - the player's avatar, and her helpers. The helpers simply step through the poses at all times, never faltering, to offer a consistent example to follow. Meanwhile, the player's avatar, which is visually distinct, reacts to whether the player has matched the expected pose within the expected time (Figure 4). 


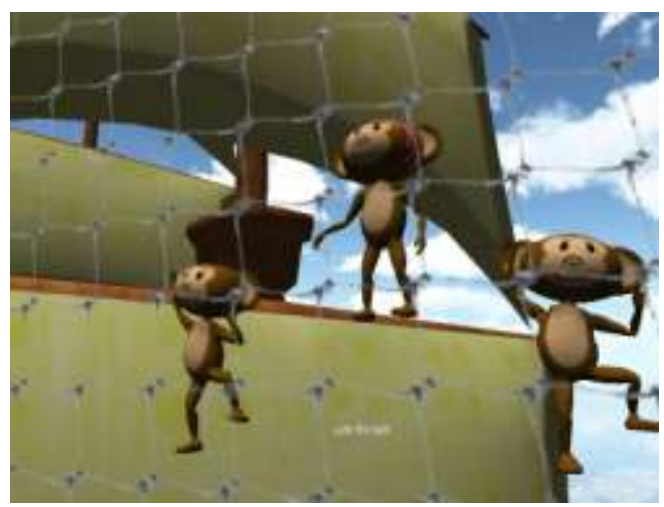

Figure 4 Players are represented by the middle character; the others provide an example to follow

\subsubsection{Pose Design}

The game involves five different exercises which are performed repetitively. Each exercise is composed of two poses. All the exercises are designed to exploit the full-body interface by demanding that the player shift his body weight.

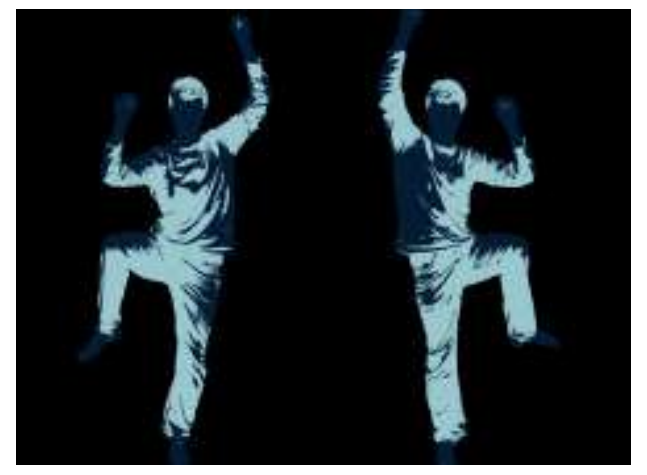

Figure 5 Pose design "Monkey Climb"

The Monkey Climb - In the context of the story, the idea of this motion is to climb a net. Players keep their arms up, and draw each knee up towards the elbow in turn.

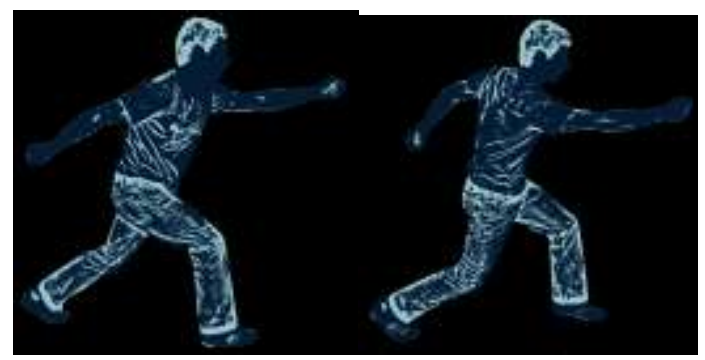

Figure 6 Pose design "Sword Hop" 
The Sword Hop - In the context of the story, the idea of this motion is that the player has a pair of swords, thrusting each of them forward in turn. The player thrusts each hand forward while moving into a stepping stance where the opposite leg is also forward. Then, the stance is switched in a single motion that can be accomplished with a small hop or step.

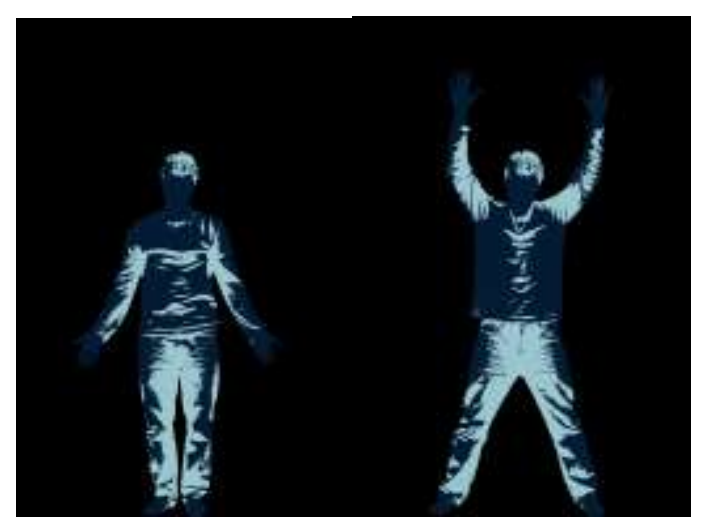

Figure 7 Pose design "Swim Jack"

The Swim Jack - In the context of the story, the idea of this motion is a backstroke through water. In practice, it is a regular jumping jack with brief pauses on each pose.

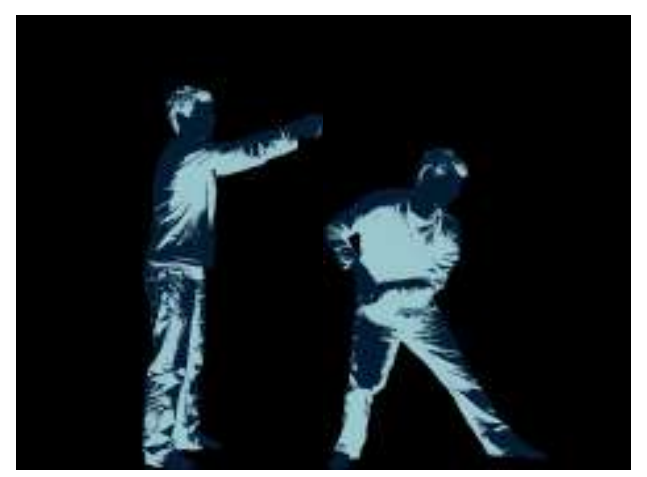

Figure 8 Pose design "Rigging Pull"

The Rigging Pull - In the context of the story, the player pulls a rope horizontally to move something tied to the rope. The player starts by standing and reaching out as if grabbing the rope, and then steps back while moving her arms to her waist as if pulling it back. Then the player returns to standing up to grab the next length of the rope. 


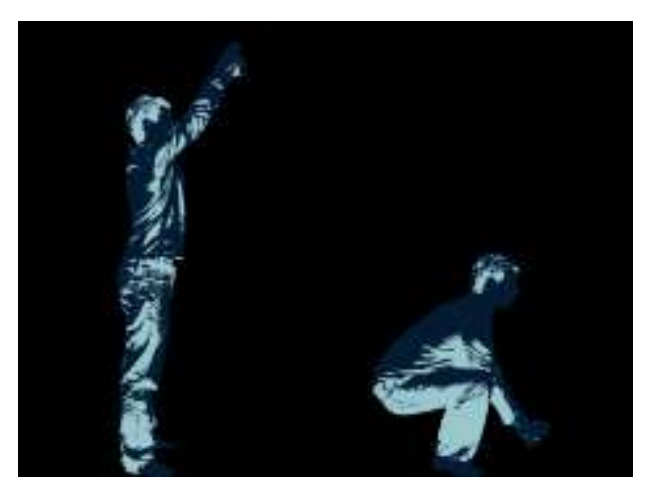

Figure 9 Pose design "Rigging Squat"

The Rigging Squat - In the context of the story, the player pulls a rope down vertically to adjust the sails of a ship. The player starts by standing and reaching up as if grabbing the rope, and then squats down and lowers their arms as if pulling it down. The player repeats by standing back up and reaching up again.

\subsubsection{Motivating Pace and Form}

Essential to the game's mechanic is motivating the player to perform the exercise with consistent pace and form. The game needs to encourage the player to follow along as closely as possible, performing the exercise correctly at all times. In this way, a player fulfills an exercise routine with consistency and effort by achieving success in the game. Several elements of the game's presentation motivate consistency:

- Visual feedback - The player's animated avatar reacts to performance on each movement. If the player is keeping up with the helper NPCs, the movement proceeds normally. If the player has not matched the pose in time, the avatar momentarily appears to stagger, trying to recover balance. This informs the player of whether the last pose was successful, allowing them to re-evaluate their form and timing if necessary.

- Audio feedback - Similar to the animated avatar's reaction, audio cues inform the player of success on each movement. A positive sound is heard on success, a tinkling bell similar to the sound heard when collecting a coin or minor item in many video games. A negative (but not harsh) sound is heard on failure, a cartoonish honking horn. The volume of these sounds is balanced such that they are distinct but not disturbing and do not drown out the background music and other effects. 
- Combo counter - As a player continues to match the poses, successive matches earn a combo score. The combo count is visualized prominently, affording the player a simple measure of the consistency of her performance. Ideally, the player wants to maximize this combo, a desire which invokes self-competition. The player naturally wants to get a high score, and from stage to stage, the player can try to maximize her combos relative to her own past performance.

The action stage proceeds, as the player attempts to maximize combos. After a set duration, the action stage is complete, and the game proceeds to another cinematic. The story proceeds, alternating between action stages and cinematic stages.

\subsubsection{Development}

Sea Monkeys was created using the Unity 4 game engine. Visual assets were created with Autodesk Maya, and cinematic sequences were created with Adobe Flash.

The SNAP system is managed using original software to process raw sensor data and decide if a pose is being performed.

During the game session, participants wear the Zephyr HxM BT heart rate monitor [75]. It is a wireless device worn about the chest with an elastic strap. It connects via Bluetooth to the PC running the game. The data received from the monitor is recorded for analysis and also used in real-time by the game. The HxM has been used in past exergaming research [2] based on validation of other devices in the same product line [74] against more sophisticated ECG devices.

Figure 10 outlines the relationship of the game, supporting software, and peripherals.

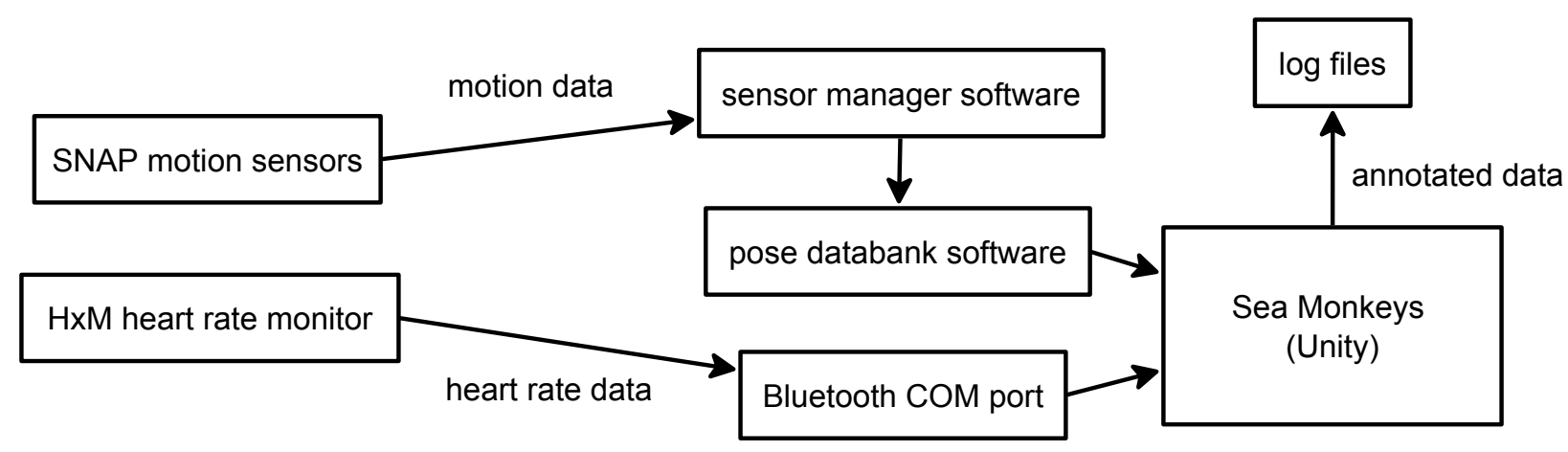

Figure 10 Hardware and software relationships supporting the game 


\subsection{Experiment Overview}

The procession of an experimental session with the game is outlined here. Following sections explore the rationale and evaluation for major design elements, including exercise measurement in section 3.3, adaptivity in section 3.4 , and story-driven design in section 3.5 .

Each session involves a single participant guided by a researcher. Although the exercise demanded by the game is not designed to be extreme, some exertion is expected, so bottled water is made readily available for participants.

1. The participant puts on all sensors, including the heart rate monitor, and the SNAP accelerometers. Connectivity with the host PC is confirmed. Game settings are adjusted, including the player's age for heart rate calculations, and whether the game will play in Adapt mode (if this participant is part of the experimental group) or Normal mode (control group). From this point on, the game is recording heart rate data, pose data, and internal state data to a log file.

2. The participant sits at rest for about ten minutes. A reading of the resting heart rate is taken. Further heart rate calculations for exercise measurement are outlined in section 3.3.

3. The researcher explains each pose and exercise. The participant practices the moves while the researcher performs data assimilation, taking several samples of accelerometer data at the moments the player performs a pose. This builds the databank used by SNAP to distinguish poses during gameplay. Meanwhile, the participant becomes accustomed to the moves, and physically warms up for the upcoming exercise. The duration of this stage can vary depending on the coordination of the researcher and the participant.

4. The participant rests briefly before beginning gameplay for 5 to 10 minutes. This helps to normalize the heart rate data still to be generated, preventing some participants from starting the game with their heart rate already significantly stimulated.

5. Gameplay begins with the opening cinematic stage. The game proceeds, alternating action stages and cinematic stages, for a total time of about 10 minutes.

a. Depending on the mode in which the game is set, adaptation for challenge management may be performed. Adaptation is detailed in section 3.4.

b. Each action stage takes about 1 minute, while each cinematic stage takes 30 to 45 seconds. Stages and the story they support are detailed in section 3.5. 
6. The participant takes a break during which she rests for a time, the duration of her choice, preparing for the second playthrough of the game.

7. The participant plays the game a second time with the same content and in the same mode (either Adapt or Normal). This results in more heart rate data, and allows more opportunity for the player to experience psychological flow, as discussed in section 3.6.1. Another 10 minutes of play time brings the total exposure to about 20 minutes plus the break.

8. With the game complete, the participant removes the sensors, rests and completes the survey.

\subsection{Invoking and Measuring Exercise}

One of the major requirements for an exergame is to provide effective physical exercise. In order to be effective, this study employs an exergame design inspired by interval training. For the purpose of testing, exercise is defined in terms of heart rate measurements gathered using a heart rate monitor.

\subsubsection{Gamified Interval Training}

An effective workout demands that the game have the capacity to elicit physical exertion from the player which is equivalent to conventional exercise. The approach taken in the current study is inspired by interval training, particularly High-Intensity Interval Training, or HIIT [29,54], a relatively recent trend in physical training.

In HIIT, a trainee will employ brief, repeating periods of highly intense exercise, with recovery periods between those peaks of intensity. From a design perspective, this style of working out is very compatible with typical game narratives. Many games which are partly or entirely linear will feature periods of elevated pace, risk and challenge, separated by periods of less intense activities such as upkeep, travelling, or advancing the plot in a more passive way. Figure 11 outlines the similarity of these patterns of action. 

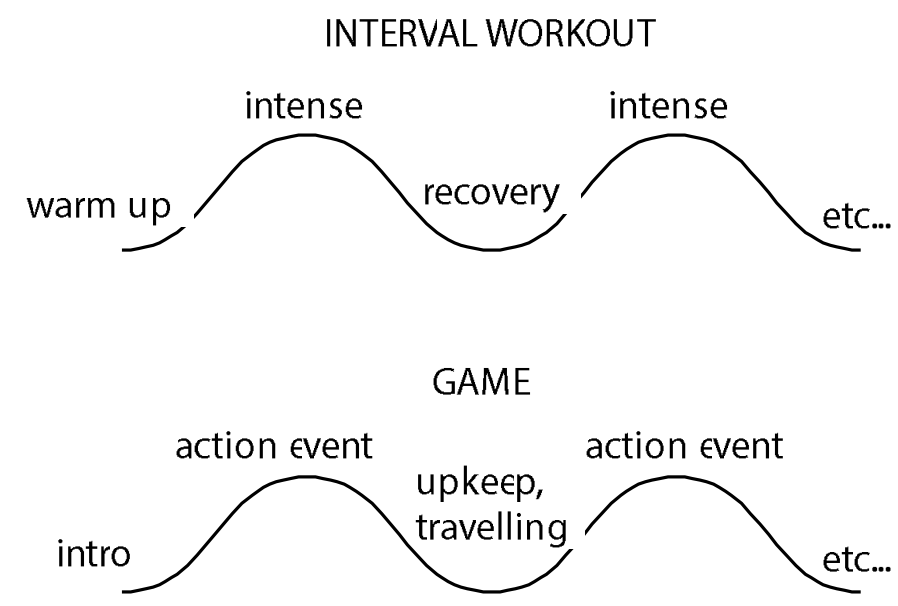

Figure 11 Simplified curves of intensity during the experiences of gaming and interval-based exercise. The similar curves indicate compatible designs, opening the door for exergames to combine them into one experience.

The current study exploits the compatibility of game narratives and interval training. The exergame puts the player through periods of high physical demand as part of the game action, and separates these periods with cinematic segments which advance the story and also allow for physical recovery. Mainstream games such as Ubisoft's Your Shape haven taken a similar approach, but have not contextualised the interval-based workout in a story. Rationale for this story-driven design is expanded in section 3.5. This section will continue to cover the invocation and measurement of exercise.

\subsubsection{Implementation}

In order to fulfill an interval-training based workout, the timing of the game is carefully controlled. The motions required on each action stage are repetitive and exertive, so they become demanding fairly quickly, and each action stage can be relatively intense. The timing is enforced so that each action stage takes one minute, and each cinematic takes between 30 and 45 seconds. In addition, each action stage begins with a 15 -second period where a tutorial animation notifies the player of which exercise is coming up, and reminds them how exactly to perform it. 
Overall, the player will spend 30 to 45 seconds watching a cinematic, followed by 15 seconds of tutorial animation, followed by 1 minute of actual action. Interval-based training is sometimes described in terms of a "time-on, time-off" ratio, or the ratio of exercise duration to rest duration. In those terms, throughout the game, the player experiences a ratio somewhere between $4: 3$ and 1:1. This is not outside the realm of interval training, although a ratio of 2:1 is often seen [54]. However, since our objective is not highintensity exercise, but rather moderate-intensity, we estimate that our ratio is appropriate. It is also an affordance for our story-driven game design.

\subsubsection{Heart Rate Terminology}

This study considers some essential aspects of heart rate (HR) measurement in order to detect cardiovascular exercise.

A person's resting heart rate is the approximate rate at which the heart beats when at rest - not undergoing any physical exertion. If exertion has occurred recently, a few minutes is generally required for a person to reach their resting heart rate.

A person's maximum heart rate is the highest physiologically possible rate at which a person's heart rate can physically beat. This value is calculated based on age, rather than being measured directly.

A person's heart rate reserve is the range between their resting and maximum heart rates. This is the range in which the heart performs during exertion. The next sections examine standards of how to establish the range, and where in the range we can expect to see exercise.

\subsubsection{Calculating Maximum Heart Rate}

Trying to directly measure an individual's true maximum heart rate is difficult. Determining a value repeatably and confidently by direct measurement presents a danger to the participant. The maximum HR value is meant to be the fastest the heart can physically beat, but actually pushing the heart to its maximal performance presents a risk of injury. Small studies such as the present one would be burdened with handling this risk.

Instead, there is a history of efforts to create a formula to calculate a person's approximate maximum HR. In order to be practical, these formulas strive to be both accurate and relatively easy to use.

A common rule-of-thumb to calculate maximum HR is: 


$$
\operatorname{HRmax}=220-\text { age }
$$

(1) Haskell \& Fox

This is often attributed to Haskell and Fox [28]. Several more recent efforts have introduced only a little more complexity in favor of accuracy. In 2001, Tanaka [56] presented this formula for general adults::

$$
\text { HRmax }=208-0.7 \times \text { age } \quad \text { (2) Tanaka }
$$

It was evaluated to be at least as good as older formulas [7]. In 2002, Robergs reviewed several formulas [42] and concluded that the most accurate (despite unnacceptably large error) came from Inbar [24]:

$$
\text { HRmax }=205.8-(0.685 \times \text { age }) \quad \text { (3) Inbar via Robergs }
$$

In 2003, Wohlfart [67] presented a formula for men:

$$
\operatorname{HRmax}=203.7 /\left(1+\mathrm{e}^{0.033 *(\operatorname{age}-104.3)}\right)
$$

(4) Wohlfart men

and a formula for women:

$$
\operatorname{HRmax}=190.2 /\left(1+\mathrm{e}^{0.0453 *(\text { age }-107.5)}\right)
$$

(5) Wohlfart women

In 2007, Gellish [15] offered several formulas for general adults, including a simple one that has been used in other exergaming research [49]:

$$
\text { HRmax }=207-(0.7 * \text { age }) \quad \text { (6) Gellish B }
$$

as well as another with a better margin of error:

$$
\text { HRmax }=192-0.007 * \operatorname{age}^{2} \quad \text { (7) Gellish A }
$$

Finally, In 2010, Gulati [20] presented a new formula for women:

$$
\text { HRmax }=206-(0.88 \times \text { age }) \quad \text { (8) Gulati women }
$$

For the purpose of the present study, the latter formula from Gellish (7) is selected. It is the most recent effort in our awareness to create a formula for general adults, and among the formulas presented in its source, it is the most recommended by its author in terms of accuracy. 
As a simple check that the selected formula seems sound, we compare these formulas in Appendix A, which lists the results of the formulas for various ages. Primarily, we find that equation (7) does not differ from the others by any clearly suspicious margin. Secondly, we find that on average, the results of the other recent formulas are within approximately +- 3 BPM of equation (7). We conclude, in the absence of a universal consensus of how to calculate maximum HR, that equation (7) is suitable for use in this study.

\subsubsection{Exercise Prescription}

Heart rate reserve (HRR) is the range from resting HR to maximum HR for an individual. In other words, it is the expected operational range of the heart. Resting HR is at $0 \% \mathrm{HRR}$, and maximum HR is at $100 \%$ HRR.

The American College of Sports Medicine (ACSM) makes recommendations for when, in terms of HRR, we can expect to see cardiovascular exercise. ACSM standards have been used in past exergaming research $[2,55,66]$. Effectively, heart rate performance is an acceptable indication of physical exercise, as shown in [66]. ACSM [59] prescribes these HRR ranges for exercise:

- $\quad$ Light exercise: $20 \%$ to $39 \%$ HRR

- Moderate exercise: $40 \%$ to $59 \%$ HRR

- Hard exercise: $60 \%$ to $84 \%$ HRR

\subsubsection{Invoking and Measuring Exercise: Evaluation}

For the purpose of this study, the main exercise goal of the game is to invoke moderate exercise. By collecting initial information about participants, including resting heart rate and age, we establish a data model reflecting cardiovascular performance. Throughout the game, we record heart date data in real-time to populate the model.

Because we implement gamified interval training, we expect at least some of the gameplay session to include heart rate results indicating exercise. The principal evaluation of this design is the amount of time a player's heart rate appears in the moderate exercise range. Combined with the adaptation as described in the next section, our exercise findings are towards investigation of Research Question \#1. 


\subsection{Adaptation for Challenge Management}

Individual people have unique levels of fitness and physical capacity. A physical task that is relatively challenging for one person because of its intensity, its duration, or the techniques required, may be relatively easy for someone with a greater level of fitness. While engaged in physical activity, whether it is conventional exercise or the gameplay of an exergame, individual users will react differently to a given task, and their experience will differ. Furthermore, the benefit to the user, in terms of both fitness and enjoyment, can be hindered if the task is outside the range of difficulty appropriate for their particular level. If the task is too easy, the user will gain little or no benefit from doing something that is not at all physically challenging, and indeed their fitness level may even degrade over time [45]. On the other hand, if the task is too difficult, benefit will also be unreachable as the user is unable to fulfill the task and thus gain the benefit of following through with the exercise.

The variety of physical fitness levels to be found in users presents a problem for exergame design: how to ensure that the benefits of physical activity are optimally promoted in as wide a spectrum of users as possible. The solution this study explores is adaptation for challenge management. When our game is set to adapt challenge, each player's performance is evaluated using heart rate standards for exercise measurement, and their performance is used to adjust the remainder of their experience.

Ideally, the result is the fulfillment of the two components of the dual-flow model, attractiveness, the appeal and enjoyment felt by the player, and effectiveness, the degree to which the exercise is an appropriate, productive workout. Both components are tied to the level of challenge experienced by the player. In our game, that challenge is presented by a single game mechanic where the player performs exercise moves which are contextualized by a story.

Our game employs challenge management to address both components of dual-flow:

- Successful use of the attractiveness component is measurable through player reports following play sessions. It results in consistent enjoyment, which, in a mainstream market context, can lead to greater player retention, and a larger player population by virtue of the game being fun over a long period.

- Successful use of the effectiveness component is measurable in the short term using heart rate data. It allows us to anticipate potential long-term results, including the maintenance or improvement of physical fitness and weight management, which can increase the chance of broader health benefits over a person's lifetime. 
Both components are served in our game by a single challenge management mechanism. We believe this represents a fully integrated gamification of exercise.

\subsubsection{Implementation}

The game is played in one of two modes: Adapt, which performs challenge management during play, or Normal, which does not. In Adapt mode, the difficulty of the game is adjusted dynamically according to feedback from the player. In Normal mode, the game remains at a default challenge level throughout play.

This study was performed using independent measures across two groups. The control group played in Normal mode, not experiencing any challenge management, and the experimental group played in Adapt mode, with the challenge level being adapted to suit them.

In Adapt mode, the game performs an adaptation at the end of each action stage, to prepare for the next action stage. The adaptation is in two parts: the evaluation of feedback from the player, and the adjustment axis whose value is changed over time. The evaluation feeds into the adjustment axis through the adaptation algorithm described in the next section. The adjustment axis feeds into the evaluation through the biometric reaction in the player, detected by the heart rate monitor. With each part feeding into the other, a feedback loop is completed.

The evaluation is in terms of heart rate performance. While playing the game, at the conclusion of an action phase is when physical effort is greatest as the player strives to endure the exercise and maintain pace and form. The game has been constantly reading the player's heart rate, and at this point, an average heart rate is calculated for the last 15 seconds (HRavg). The value of HRavg is the heart rate that this action stage has managed to push the user towards; it is the measure of the exertion demanded by this action stage. It is calculated by taking the mean of the last 15 samples received from the heart rate monitor, where a sample is received about once per second.

The adjustment axis is the pace of the exercise. Each different exercise has its own range of possible pace, between a minimum (which should be fairly slow for most people), and a maximum (which should be rather quick for most people). The bounds of these ranges were chosen through informal preliminary testing.

The pace of an exercise is effectively its level of difficulty. In the game, a more difficult exercise is one that the player is expected to perform faster, while the expectations remain the same for accuracy of form and timing. 


\subsubsection{Algorithm}

In order to adjust the challenge level of the game, an adaptation algorithm is used. The algorithm involves both static and dynamic data on the player. The result is the game's difficulty (the pace of exercise) being modified to a new value within an allowed range.

The adaptation algorithm uses static heart rate information about the individual player, including her resting heart rate and her maximum heart rate. Before the game is played, the player's resting heart rate (HRrest) is measured manually by the researcher. This is done by having the player sit at rest for about ten minutes. The maximum heart rate (HRmax) is calculated based on the player's age, using a formula proposed by Gellish [15] (section 3.3.4).

The player's target heart rate (HRtarget) is the ideal heart rate that the game should strive to invoke through challenge management (when played in Adapt mode). It is calculated according to the ACSM prescriptions for measuring exercise using heart rate reserve (HRR) [59] as described in section 3.3.5. For the purpose of this study, the target for players was exercise of a moderate intensity, $40 \%$ to $59 \% \mathrm{HRR}$. The game seeks to ensure that players attain maximum time spent in this range by pushing them towards the centre of it, $50 \%$ HRR. Therefore, the target heart rate is the HR equivalent of an HRR of $50 \%$ intensity:

$$
\text { HRtarget }=((\text { HRmax }- \text { HRrest }) * \text { intensity })+\text { HRrest. }
$$

The game's current difficulty setting is its current position within the range of possible pace for each exercise. The position in that range is a single percentage figure, Pace $\%$. For instance, with a Pace $\%$ of zero, the exercise proceeds the slowest it is allowed to go, and is thus the easiest it can be. With a Pace\% of 100 , the game proceeds the fastest, and is thus the most difficult it can be.

The algorithm maps the player's current HRavg to a position along a curve. The value of the curve is the amount by which the difficulty is adjusted. That amount is added to the current Pace\%, making the game harder or easier by some amount. The shape and bounds of the curve depend on whether the player's current HRavg is below or above HRtarget, i.e. whether she is currently below or above the ideal heart rate.

When below HRtarget, the HRavg is mapped to a range between HRrest and HRtarget. The position of HRavg within that range is placed on a curve (Figure 12):

$$
\Delta \mathrm{Pace} \%=(\mathrm{x}-1)^{2}
$$




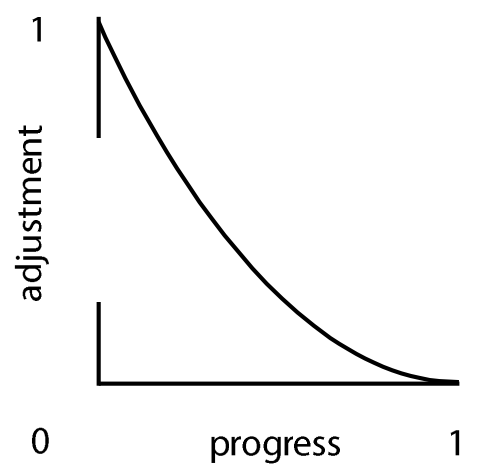

Figure 12 The adjustment curve used by algorithm in the case where the player is below HRtarget. The progress axis represents the player's current position in the range from HRrest to HRtarget.

When above HRtarget, the HRavg is mapped to a range between HRtarget and HRmax. The position of HRavg with that range is placed on a curve (Figure 13):

$$
\Delta \mathrm{Pace} \%=\mathrm{x}^{2}
$$

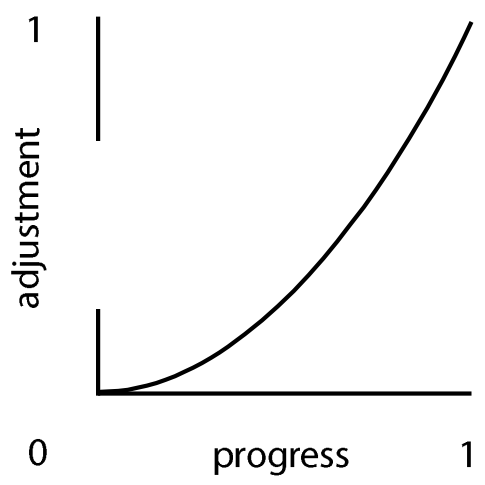

Figure 13 The adjustment curve used by algorithm in the case where the player is above HRtarget. The progress axis represents the player's current position in the range from HRtarget to HRmax.

The value of the curve (which can be from zero to 1 ) is converted to the percentage by which Pace\% should be adjusted. If the player is below HRtarget, we want to increase the difficulty to push the player up towards the target, so we add that percentage to Pace\%. If the player is above the target, then we want to allow the player a slight reprieve so that we do not exceed our target intensity and risk physical failure through excess difficulty. In that case, we subtract the percentage from Pace\%. After this adjustment, the Pace $\%$ itself is clamped to a range of $0 \%$ to $100 \%$. We never want to go slower (less than $0 \%$ ) or faster (more than 100\%) than the limits we have manually set. 
The algorithm covers the extreme cases for adaptation. One extreme case is, after an action stage, the player has made no progress away from their HRrest and towards HRtarget, meaning the exercise has not resulted in any apparent exertion. In this case, the algorithm adjusts challenge by an increase of $\Delta \mathrm{Pace} \%$ $=100 \%$, increasing the challenge to maximum in order to promote exertion in this apparently very fit player. The other extreme case is, after an action stage, the player is close to HRmax. This is far more exertion than necessary for most exercise routines, much less our moderate-intensity one. In this case, the algorithm adjusts challenge by a decrease of $\Delta \mathrm{Pace} \%=100 \%$, decreasing the challenge to minimum to suit this already very overworked player.

The shapes of the curves are designed so that their shapes result in certain behavior. Both are very steep at the ends where the player is farthest from the target, and both are very shallow at the ends where the player is close to the target. As a result, the adjustment is greater where we are nearer to the extreme cases. Where we are close to the target, the adjustment is less, allowing us to slowly and carefully adjust towards an optimum challenge. This helps to prevent overshooting the target by making the game too challenging where before it was a bit too easy, or undershooting the target by making it too easy where before it was a bit too challenging.

\subsubsection{Background Rationale}

The adaptation algorithm is inspired largely by Baradoy's DanceBeat project [2], which also reacted to a player's heart rate performance in order to adjust the pace, and thus the challenge, of an exergame. The usage of curves to smooth towards a target is inspired by Stach's Heart Burn game [49].

The algorithm is able to adapt the game to better suit players because it employs a player model, a data structure representing relevant aspects of the player. Smith offers a taxonomy of player modelling [48], suggesting an organized series of qualifiers which can concisely and comprehensively describe a player modelling application relative to others.

Our game can be described in the terms of that taxonomy in order to position relative to other adaptivity research. It is "individual" in scope, serving each player based on their own input, real-time heart rate data. It is "generative" in purpose, directly producing precise detail for gameplay, the pace of exercise. Its domain is "human reactions", focusing on an invoked reaction in the player, heart rate, in order to make decisions. Its source is "synthetic", relying on concepts external to the game to inform its design and procedure; specifically it relies on theories and research practices of psychological flow, as well as ACSM recommendations for exercise measurement. These concepts do not need to enter the player's understanding for the adaptivity to function. 


\subsubsection{Adaptation for Challenge Management: Evaluation}

The game's implementation of exercise moves generally resembles many fitness routines. It is expected to be generally adequate to challenge players. However, optimizing the experience of each player stands to have a positive effect for individuals.

We evaluate this effect by comparing the results of our two participant groups - the experimental group, playing in Adapt mode, and the control group, playing in Normal mode. Notable differences between the results of these groups can be correlated with the experimental condition: the presence or absence of adaptation during gameplay. These correlations are towards investigating Research Question \#1.

\subsection{Story-Driven Exergaming}

One of the major requirements for an exergame is to engage the player in an attractive experience. In order to attract players, our game contextualizes the exercise routine with a continuous story. Over the course of a series of cinematic stages and action stages, the player character interacts with others around him in a struggle for survival.

The cinematic stages are animated and use sound effects. Dialogue is not used in order to simplify the presentation and avert language barriers. In order to produce a compelling amount of high quality content, they are presented in a simple format: a motion comic. Cinematic stages are composed of mostly still frames with occasional movement of objects.

\subsubsection{Gameplay Stages}

The game's story proceeds over the course of the stages as follows. The exercise moves are described in section 3.1.4.

1. Cinematic - The protagonist and player character is introduced, a monkey sailor named Jacques, wearing a bandana. Also introduced is his companion parrot, Francesco. They are aboard the ship Bearded Guppy. Looking through his spyglass, Jacques spots a ship flying an enemy pirate flag bearing down on them. He springs into action, leaping onto the Guppy's rigging.

2. Action - Jacques climbs up the rigging. Players perform a climbing motion, the Monkey Climb.

3. Cinematic - Reaching the top, Jacques unfurls the flag, preparing the ship for confrontation. The Captain appears and orders the sails be trimmed. Jacques jumps down to help. 
4. Action - Jacques pulls ropes to manipulate the sails. Players perform a squatting action to mimic a large bodily pulling motion, the Rigging Squat.

5. Cinematic - The sails are set. The enemy ship fires a cannonball which shakes the deck and spills the Captain overboard. Jacques throws a rope down after him and prepares to pull him up.

6. Action - Jacques pulls the captain back onto the ship. Players perform a stepping action to mimic a large bodily pulling motion, the Rigging Pull.

7. Cinematic - The captain is rescued. Meanwhile, the enemy ship has closed, and enemy crocodiles are boarding the Guppy. Jacques, Francesco, and all deckhands prepare to repel boarders.

8. Action - A sword battle takes place. Players perform alternate between two poses designed to mildly test coordination, as if fighting with a sword in each hand, the Sword Hop.

9. Cinematic - A special enemy appears - the crocodile captain Maurice. He swings over, kicks Jacques overboard, and captures Francesco.

10. Action - Left behind, Jacques swims for shore. Players perform a jumping-jack-like action to mimic a backstroke, the Swim Jack.

11. Cinematic - Jacques reaches a remote island. He contemplates how to find Maurice and rescue his pet. Here, the game is complete, in a "to be continued" ending.

\subsubsection{Hiding Exercise in Plain Sight}

In the DanceBeat project, Baradoy [2] recommends that the mechanics of an exergame should be designed so that the desired physical exertion results from achieving the in-game goals. This supports the gamification of exercise, but there is an important distinction to be made in terms of how the user perceives their in-game achievement and the resulting exercise. To what degree should the user be aware of the exercise involved in the game?

A major demand for exergames is to fulfill fitness needs for individuals who are averse to conventional exercise methods. As a result, it is tempting to approach exergame design from the standpoint that the exercise aspect should be concealed in some way. A designer's intent could be for the player to engage with an exergame without realising the exercise aspect involved. However, not only is such concealment or deception ethically questionable [73], in the long run it is unnecessarily restrictive. 
We can consider multiple stages of awareness during the player's total experience of an exergame. In advance of gameplay, the potential player is in some situation that leads to the decision to play, such as making a purchase, or selecting a game from an existing collection. Later, when gameplay has commenced, the player is interacting with the game, engaging with the content. In the pre-gameplay stage, it is not desirable for an exergame to conceal its nature as a fitness solution that involves physical exercise. Fitness benefit, the core service that has been gamified, is not unto itself repulsive to players. For instance, Wii Fit was marketed primarily on such benefit, and was very successful commercially [39]. An exergame can make itself known for its true nature, as both entertainment and fitness are selling points.

In the gameplay stage, we can ask the question again: what should the player's level of awareness of the exercise be? At this point, the player has reached the point where exercise is most arduous - actually performing it. However, we propose that even at this point, presenting the exercise for what it is does not disrupt enjoyment, as long as it has been integrated properly with the game elements of the experience. In other words, if a robust gamification has taken place, and both components of dual-flow are fulfilled, then the player is engaged with the game while maintaining awareness of the exercise, experiencing both enjoyment and a productive workout.

In contrast, existing exergames do not tend to take this approach. Usually, they either focus on more on the game context, or more on the fitness context. For instance, Ubisoft's Marvel Avengers for play with the Microsoft Kinect utilizes characters which are familiar to fans and employs game mechanics involving highly contextualised motions. For example, Hulk has ground-smashing moves that are invoked by dramatic arm motions that emulate the result. Here, the focus is on the game context. Meanwhile, Ubisoft's Your Shape series offers a full featured game experience that hardly ever departs from the context of a workout session. Here, the focus is more on the fitness context.

The alternative to presenting the workout for what it is during gameplay is to conceal it in some way, drawing attention away from the fitness aspects of the game. While it is important to focus the player's attention on game aspects, we believe that it is unnecessary to approach exergame design expecting to totally remove the player's awareness of exercise. So long as the exertion demanded by the game constitutes an effective workout, the user will inevitably realise that they are breathing hard, working their muscles, maintaining a pace, and so on. 
Instead of designing against the player's awareness of exercising, integrating that awareness into the game design may help to promote exercise habits. It has been seen that an increased awareness of one's level of exercise can increase willingness to play exergames [61], and this relationship may go in the other direction as well. With this approach, a well-integrated workout is presented as part of an engaging game, rather than being concealed by it. The player enjoys the game either way and, having been conscious of a satisfying exercise experience, may be emboldened to pursue exercise in other forms. We refer to this approach as hiding the exercise "in plain sight".

\subsubsection{Story-Driven Exergaming: Evaluation}

In our game, the story exhibits continuity over multiple stages in a drawn-out narrative rising action. We suspect that this continuity can promote engagement, where players become emotionally invested in the progress and difficulties of the character who serves as their avatar. Pursuant to this suspicion, the story in its current form has been designed with an open ending, without a final resolution. We propose that this will generate interest in a continuation of the story.

Hiding the exercise component of our exergame "in plain sight", as described in the previous section, entails supporting the player's awareness of the fact that they are participating in a workout, while supplying them with content to engage with at the same time. We propose to evaluate the validity of this approach by generating player interest and investment in the story, in the context of our exergame which promotes, rather than suppress, workout awareness. While the player's investment is driven by the story, their awareness of the workout is not discouraged. Because the two aspects are integrated, the investment is in the exergaming experience overall. In the long term, this could hopefully lead to the realization that exercise can be fun when contextualized within the story, as well as in other contexts such as conventional exercise alone and with others.

During the study, informal player comments have supported these propositions. We investigate the propositions formally using questions on the survey in Appendix B. The survey includes direct, original questions, as well as the Flow State Scale [26] in order to allow participants to report experiences of enjoyment, performing exercise, and psychological flow. These reports will be towards investigation of Research Question \#2. 


\subsection{Fulfilling Design Requirements and Addressing Limitations}

In the Background, we examined two sets of requirements that can help our exergame design. One is an original compilation of lessons in promoting flow experiences, gleaned from our review of flow studies. The other is a list of recommendations for general exergame design from [71]. We fulfill these requirements to various degrees depending on their applicability to the study.

\subsubsection{Invoking and Measuring Flow}

Both physical exercise [51,57,62] and video gaming [53] have been seen as conducive to flow. Exergames exploit this compatibility, delivering a flow-conducive experience which combines those activities. In this endeavor, we measure the degree of success of our game using the Flow State Scale [26], a general-purpose survey instrument. The phrasing of its questions has been modified very slightly to be compatible with our study; see Appendix B.

In order to invoke flow, our game design considers the flow lessons outlined from the review in section 2.1.3. Considering these lessons means we should avoid some shortcomings and capitalize on some strengths of past studies. We have been able to address those lessons to various degrees in the following ways.

1. Allow sufficient time. Ten minutes or less is problematic. While a minimum safe period is not known, an hour has been recommended as quite sufficient. Generally, the longer the period, the better the chance of flow experience. In practice, however, we must consider constraints for our study. These include the physical endurance of participants in the exergame, how much time we can ask of participants while still expecting an acceptable response rate, and how much content we can make available for the game's continuous story. A single playthrough of our game takes about 11 minutes. In our experimental sessions, we have asked players to play the game twice (for a total of 22 minutes, plus a rest between the two plays). Given our constraints, this is how we afford maximum available opportunity for flow experience.

2. Use everyday language when asking participants about their experiences. The survey instrument uses plain, easy language to ask about fun, physical effort, and past experiences. It incorporates the Flow State Scale [26], which also accords with this lesson.

3. Do not neglect ACT, LOSS and TRAN. While challenging to measure and interpret by self-report, these remain important components of classic flow. Employing the Flow State Scale comprehensively examines these and all other flow components. 
4. If possible, mix self reports with objective measures. This is a principal design feature of this study, gathering data from the game and the heart rate monitor, and also employing a survey.

5. If possible, use more than one framework. While the design of the game, and the study itself, is motivated in large part by the dual-flow model [45], we draw inspiration also from service-based gamification. As well, the results are gathered and interpreted using the Flow State Scale. Performing the study by employing these frameworks results in a more nuanced perspective on the design and value of exergames.

Asking participants to play through two rounds of the game using the same content is a limitation of the study. It is a resort based on a limited capacity to produce content in order to allow sufficient time for flow experiences as noted above. Ideally, a greater labor capacity and more efficient content pipeline could support one continuous experience with no repeating content. Future expansions to the study should prioritize this concern.

The break between the two rounds may also be noted as another limitation on promoting flow experiences, since it interrupts the continuity of the experience. However, given the intensity of our interval-based exercise stages, we find that taking a break is physically appropriate to allow players to recover before more play. This study seeks to validate the interval-training-based, story-driven format for exergames. Future studies might adopt this format while employing a greater level of exercise prescription expertise in order to surmount the need for a prolonged break, or fill the break with a much less intense secondary game activity, if appropriate according to that expertise.

\subsubsection{Exergame Design Practices}

The game fulfills many of Yim's [71] recommendations for exergame design outlined in section 2.1. Two of those recommendations pertain to multiplayer aspects, and are thus not directly applicable to our exclusively single-player game. The other recommendations are fulfilled to varying degrees:

- Integrate music. During action stages, the game plays music which is themed to the story. There are two tracks with different moods in order to set the atmosphere of each stage according to story events. The pace of the music is adjusted by pitch-shifting to approximately match the pace of the exercise. 
- Facilitate leadership for novice players. The range of this study is very short term - participants have little time to practice, relative to a marketed game they could take home and replay. Each stage features a different exercise which the participants, as players, must put into practice for the first time. Leadership for the player is afforded by visual input of NPC helper characters. Throughout action stages, these characters constantly demonstrate a perfect example of the exercise for the player to follow. Combined with the audio feedback and combo mechanic, these characters guide the player to a high score and a productive workout. Note that although the player is lead by these characters in terms of game mechanics, the player's avatar is not a follower in the story. Rather, the protagonist is seen to be bold and outgoing in the cinematic stages, demonstrating that leadership and guidance for the player does not interfere with an empowering narrative aspect.

- Provide achievable short-term goals. The interval-based exercise modus of the game ensures that the player's effort is demanded in brief but meaningful segments. Although the game's story is continuous throughout play, it is told in small digestible segments where the player understands at any given moment what the protagonist's motivations are. Also, the motivation to maximize combo score within the allotted duration of each action sequence provides a series of short, manageable challenges that fit together in the broader context of the story.

- Hide player's fitness level. The player's performance is reflected back by the game only in the form of very abstract feedback (section 3.1.5). The player's overall fitness level is not visualized or judged directly in gameplay.

\subsubsection{Gathering Robust Experiential Reports}

The experiential aspects of the present study rely on the survey instrument (Appendix B) which incorporates the Flow State Scale (FSS). The survey is a series of seven-point Likert scale questions. Statements are to be matched to a response value from the key shown in Table 1.

Table 1 Value Key for Likert Responses

\begin{tabular}{|l|l|l|l|l|l|l|l|}
\hline Value & 1 & 2 & 3 & 4 & 5 & 6 & 7 \\
\hline $\begin{array}{l}\text { Associated } \\
\text { Response }\end{array}$ & $\begin{array}{l}\text { strongly } \\
\text { disagree }\end{array}$ & disagree & $\begin{array}{l}\text { somewhat } \\
\text { disagree }\end{array}$ & $\begin{array}{l}\text { neither } \\
\text { agree or } \\
\text { disagree }\end{array}$ & $\begin{array}{l}\text { somewhat } \\
\text { agree }\end{array}$ & agree & $\begin{array}{l}\text { strongly } \\
\text { agree }\end{array}$ \\
\hline
\end{tabular}


Reliance on self-report instruments has drawbacks which were noted in section 2.1.2.2. To address these concerns, we have taken precautions while designing our questions.

For our original questions, particularly experiential ones such as "the game was straightforward" were matched with inverted versions such as "the game was confusing". When responses are analysed, inverted questions are reversed (i.e. on a scale of 1 to 7, a response of 3 becomes a 5). These inverted questions avoid monotony during the survey that could lead participants to select responses carelessly. Instead of a survey where all questions ask if the game was good in some way, the inclusion of inverted questions creates a survey that asks if it was good and if it was bad alternately.

The FSS does not employ inverted questions, but has a similar precaution built in. Each component of flow is addressed with no less than four separate questions using different phrasing. Even if a participant understands a question differently than the researcher intended, several other questions are there to compensate. This redundancy avoids a dependency on a single response to what can be sophisticated experiential aspects.

When the survey is presented to participants, the ordering of all questions is randomized. Related questions are not grouped together, helping to avoid carryover when participants are answering. Redundant questions are not grouped together, helping to mask their redundancy. Questions are not seen together with their inverted versions, helping to mask this inversion.

With these precautions, we are confident that our survey yields meaningful results.

In order to gain insight on our survey responses, we have subjected them to t-tests and f-tests. It may be noted that, in general principle, these tests are not especially applicable to Likert responses. However, they served the purpose of this study since the questions were of seven points (better than the typical five) and the results were of an appropriate distribution.

\subsection{Recruitment}

A six-week period was allocated to perform the study. The study was advertised graphically in print, through email and through social media (Appendix C). The study was promoted in the online edition of the school paper. Anyone of adult age was welcome to participate at their own discretion. 
Several sources of participants were tapped. Individuals close at hand were encouraged to participate.

Participants in past studies within the department were contacted. Faculty, staff and students were notified by email and encouraged to share further. Some undergraduate classes were offered some course credit in exchange for participation.

An online form was set up to streamline recruitment by allowing participants to select any of a series of available timeslots. The time slots offered were one hour periods spanning approximately nine hours each day for most of the six-week period. Even after selecting a timeslot, participants were welcome to reschedule at their convenience.

Before the session, the participant would sign a consent form (Appendix D). 


\section{Results}

A sample of 25 people participated in the study by playing the game and completing the survey. 16 were male and 9 were female. Ages ranged from 18 to 36 years old. These included Carleton University students and other people in Ottawa.

Sessions took place with individuals participating alone. Participants were randomly assigned into two groups for independent measures testing. The experimental group played the game in Adapt mode, experiencing the game with adaptation for challenge management. The control group played the game in Normal mode, experiencing a non-adjusted default level of challenge. Both groups completed the same survey (Appendix B).

Participants report for themselves a wide range of regularity of exercise. On average, neither group reports themselves as outstandingly athletic, nor particularly sedentary. When asked if they get significant regular exercise, the averages response was 4.1 for Adapt group and 3.6 for Normal group, in a Likert range of 1 to 7 . Therefore we are not concerned about skewed results due to significant discrepancy in physical ability.

\subsection{Analysis}

The game recorded data on the player's heart rate, as well as internal state data, throughout the play sessions. Interpretation of the data and the survey results leads to several findings.

\subsubsection{Adaptation is Suggested to Increase Workout Reliability}

Research Question \#1 asked about the effect of individual adaptivity for challenge management on the player's exergaming experience. Our answer is that there is a suggestion of increased reliability, derived primarily from a difference between the two groups in the amount of time spent in the target exercise zone.

Throughout the game, the participant's heart rate is monitored. The total duration of the game is the time taken to proceed through all gameplay stages, which involves watching all cinematic stages and playing all action stages. 
Each participant achieves a 'target time percent'. This is the percentage of the duration of their play session during which their heart rate fell within the target zone, the "moderate" zone prescribed by the ASCM, as discussed in section 3.3.5. For this percentage of the session, the participant is considered to be achieving moderate-intensity cardiovascular exercise.

Towards Research Question \#1, the primary measurement effected by the condition of adaptation was expected to be the average target time percent. Overall, the average target time percent for the experimental group (the Adapt group, 0.31, std dev 0.09) was not considerably different than the control group (the Normal group, 0.35, std dev 0.12). Both groups experienced about the same moderate-intensity workout. This is not unexpected, as the normal, non-adaptive game experience is designed to be a serviceable "default" challenge, not necessarily worse than the adaptive experience on average.

However, in post-hoc analysis, the variance of target time percent was found to be considerably lower for the Adapt group (0.0086) than for the Normal group (0.0166). We interpret this to mean that with adaptation, the workout was more consistent between users. Although significance is not seen (we suspect due to sample size, p-value from f-test is 0.28 ), this may support the validity of our adaptation technique for challenge management in an exergame: with adaptation, any given user of the game may be more likely to receive the expected level of challenge relative to their skills. We describe this as a 'suggestion' of increased exergaming reliability in terms of dual-flow effectiveness, since the workouts seem to be more consistent.

Increased consistency would make the game more reliable as a fitness solution by fulfilling the effectiveness component of the dual-flow model, balancing physical demand against the player's fitness level. It would also make the game a more reliable source of entertainment by fulfilling the attractiveness component of the dual-flow model, balancing the demand of the game mechanic against the player's abilities. With the present sample size the study was able to attract, the results cannot definitively show this with regards to statistical significance, but we believe a larger study is worthwhile to confirm the suggestion of these results.

Increased reliability is suggested by other variance measurements of the exercise data as well. Notable results from heart rate data are presented in Table 2. Where the variance is lower for the Adapt group, the workout received with adaptation appears to be more consistent between participants, and the idea that adaptivity improves workout reliability is supported. For p-values, t-tests are performed for averages, while f-tests are performed for variances. 
Results for player success rate also evince a more consistent experience for the Adapt group. A player's success rate is the proportion of action steps he was able to match in time; it is a measure of how well the exercise was actually performed according to motion sensor data. Similar to the heart rate results, the groups saw similar averages, but considerably different variances. The Adapt group's variance was lower to a degree approaching significance (p-value 0.065 , where we would like to see below 0.05 for significance). The full-body motion input data suggests that the challenge encountered by the Adapt group was of a more reliable level between individuals.

In addition to those results from the objective data, players subjectively reported on the survey about the perceived physical demand more consistently in the experimental group than the Normal group (variances 1.28 to 2.1$)$. 
Table 2 Variance of Time Participants Spent in Exercise Ranges

\begin{tabular}{|c|c|c|c|c|}
\hline $\begin{array}{l}\text { Game } \\
\text { Timeframe }\end{array}$ & Exercise Range & $\begin{array}{l}\text { Adapt Group } \\
\text { Variance of } \\
\text { Time Spent } \\
\text { in Range }\end{array}$ & $\begin{array}{l}\text { Normal } \\
\text { Group } \\
\text { Variance of } \\
\text { Time Spent } \\
\text { in Range } \\
\end{array}$ & $\begin{array}{l}\text { Suggests Increased Reliability With } \\
\text { Adaptation? }\end{array}$ \\
\hline \multirow[t]{5}{*}{$\begin{array}{l}\text { Entire } \\
\text { session }\end{array}$} & $\begin{array}{l}\text { Target (moderate) HRR } \\
\text { zone }\end{array}$ & 0.0086 & 0.0166 & $\begin{array}{l}\text { Yes by order of magnitude } \\
\text { ( } p \text {-value } 0.28 \text { ) }\end{array}$ \\
\hline & Lower half of target zone & 0.0063 & 0.0084 & Marginally ( $p$-value 0.65 ) \\
\hline & Upper half of target zone & 0.0028 & 0.0030 & Not considerably ( $p$-value 0.96 ) \\
\hline & High HRR zone & 0.0198 & 0.0349 & Yes (p-value 0.36$)$ \\
\hline & Moderate \& high HRR & 0.0318 & 0.0368 & Marginally (p-value 0.82) \\
\hline \multirow[t]{5}{*}{$\begin{array}{l}\text { First play- } \\
\text { through }\end{array}$} & $\begin{array}{l}\text { Target (moderate) HRR } \\
\text { zone }\end{array}$ & 0.0124 & 0.0224 & Yes (p-value 0.34) \\
\hline & Lower half of target zone & 0.0127 & 0.0110 & No (p-value 0.80$)$ \\
\hline & Upper half of target zone & 0.0051 & 0.0052 & Not considerably ( $p$-value 0.99) \\
\hline & High HRR zone & 0.0344 & 0.0519 & Marginally (p-value 0.50$)$ \\
\hline & Moderate \& high HRR & 0.0460 & 0.0504 & Not considerably ( $p$-value 0.88 ) \\
\hline \multirow[t]{5}{*}{ Rest Period } & $\begin{array}{l}\text { Target (moderate) HRR } \\
\text { zone }\end{array}$ & 0.0029 & 0.0225 & $\begin{array}{l}\text { Yes, with significance } \\
(p \text {-value } 0.002)\end{array}$ \\
\hline & Lower half of target zone & 0.0022 & 0.0109 & $\begin{array}{l}\text { Yes, with significance } \\
\text { (p-value 0.01) }\end{array}$ \\
\hline & Upper half of target zone & 0.0003 & 0.0028 & $\begin{array}{l}\text { Yes, with significance } \\
\text { (p-value } 0.0003)\end{array}$ \\
\hline & High HRR zone & 0.0011 & 0.0081 & $\begin{array}{l}\text { Yes, with significance } \\
\text { (p-value } 0.002 \text { ) }\end{array}$ \\
\hline & Moderate \& high HRR & 0.0058 & 0.0511 & $\begin{array}{l}\text { Yes, with significance } \\
\text { (p-value } 0.001)\end{array}$ \\
\hline \multirow{5}{*}{$\begin{array}{l}\text { Second } \\
\text { play- } \\
\text { through }\end{array}$} & $\begin{array}{l}\text { Target (moderate) HRR } \\
\text { zone }\end{array}$ & 0.0218 & 0.0330 & Marginally ( $p$-value 0.50 ) \\
\hline & Lower half of target zone & 0.0092 & 0.0160 & $\begin{array}{l}\text { Yes by order of magnitude ( } p \text {-value } \\
0.37 \text { ) }\end{array}$ \\
\hline & Upper half of target zone & 0.0086 & 0.0079 & No (p-value 0.88$)$ \\
\hline & High HRR zone & 0.0293 & 0.0578 & Yes (p-value 0.27) \\
\hline & Moderate \& high HRR & 0.0631 & 0.0674 & Not considerably ( $p$-value 0.92 ) \\
\hline
\end{tabular}

\subsubsection{Story-Driven Exergaming Can Promote Flow and Enjoyment}

Research Question \#2 asked how enjoyable the game is, with regards to its format as a workout experience integrated as a story-driven game. Our response is that our game was enjoyed, and therefore we believe the story-driven approach to exergame design is viable. The primary measurement of this answer were the survey results from the whole pool of participants. 
Enjoyment or fun is difficult to reliably define, let alone measure [35]. To approach it in a robust way, we look for indication of psychological flow experiences during play using the Flow State Scale (FSS). Among the aspects of flow experience are autotelic enjoyment of the task, and satisfaction overall with the experience, so indications of flow help us to detect enjoyment.

These measurements inform us of the degree to which story-driven game design can help to fulfill dualflow attractiveness. We evaluate average (mean) results and standard deviations for the whole pool of participants, and also look for differences between groups by comparing those averages.

A subset of results data is highlighted throughout this description of results. A detailed summary of survey results is available in Appendix E.

FSS results show an indication of flow overall at an average of $5.2(\operatorname{std} \operatorname{dev} 0.5)$ in a Likert range of 1 to 7. We find this to be a fairly strong indication of flow experience, given our limited timeframe discussed in section 3.6.1.

The weakest flow component was TRAN, the transformation of time, speeding up or slowing down (average 3.9 overall, std dev 1.1, fairly similar across the two groups). This component describes a warping of perception relative to reality, and therefore it can be expected that TRAN experiences are not easy to obtain quickly and without significant immersion. Therefore the low rating for this component is not surprising, given our game's learning curve, as well as our limitation on timeframe.

The strongest flow component was GOAL, clear awareness of goals (average 5.86 overall, std dev 0.9, fairly similar across the two groups). This is also unsurprising given our game's uncomplicated rhythmfollowing mechanic. It accords with the absence of cognitive obstacles reported in section 4.1.1.

Enjoyment in particular was strongly indicated. The FSS results for ENJY (autotelic enjoyment) were positive (average 5.32, std dev 0.9). When asked by our direct question (not from the FSS) about whether the game was fun, the response was strongly positive (average 5.9, std dev 0.9). It was entertaining, holding the player's attention rather than becoming boring (average 5.7, std dev 0.7).

Other direct questions revealed success for the story. The game's story was helpful for the player to exercise, rather than being a distraction (average 5.9, std dev 1.2). Players indicated a fairly positive interest in a sequel to continue the story (average 5.9, std dev 1.4). 


\subsubsection{Verification of Interval Training Intensity}

The particular exercise moves used in the present study (section 3.1.4) are not directly founded on previous research. They are based on our design sensibility, common sense regarding exercise, and some trial and error in preliminary tests during development. They are primarily meant to be contextualized within the game's story while emulating the style of typical workout moves. The results demonstrate that these moves are viable for exergaming and resulted in meaningful exertion for players.

Heart rate results are used to examine the effectiveness of the game in terms of the dual-flow framework. Heart rate data from play sessions conformed to the basic outline of an interval training session, and showed the achievement of effective exercise for a considerable timeframe. Throughout the game, players spent considerable time in the moderate intensity heart rate reserve (HRR) zone, which is our primary target, and also some time in the high intensity zone. From one level to the next, these amounts generally increase as the game goes on, showing players increased exertion over time.

Figure 14 shows the escalation of player effort in terms of HRR as an average across all players in each action stage, averaged across both rounds of play. This illustrates how meaningful exercise was achieved by participants by showing the average target time percent, defined in section 4.1.1, as the reading for the moderate HRR zone. The remainder of the player's time in the stage is divided between being above or below that moderate zone. Each target time percent to be averaged is obtained separately for each player, since each player has their own target zone according to their age. Age determines theoretical maximum heart rate, and therefore the parameters of a heart rate response zone, as described in section 3.3. 


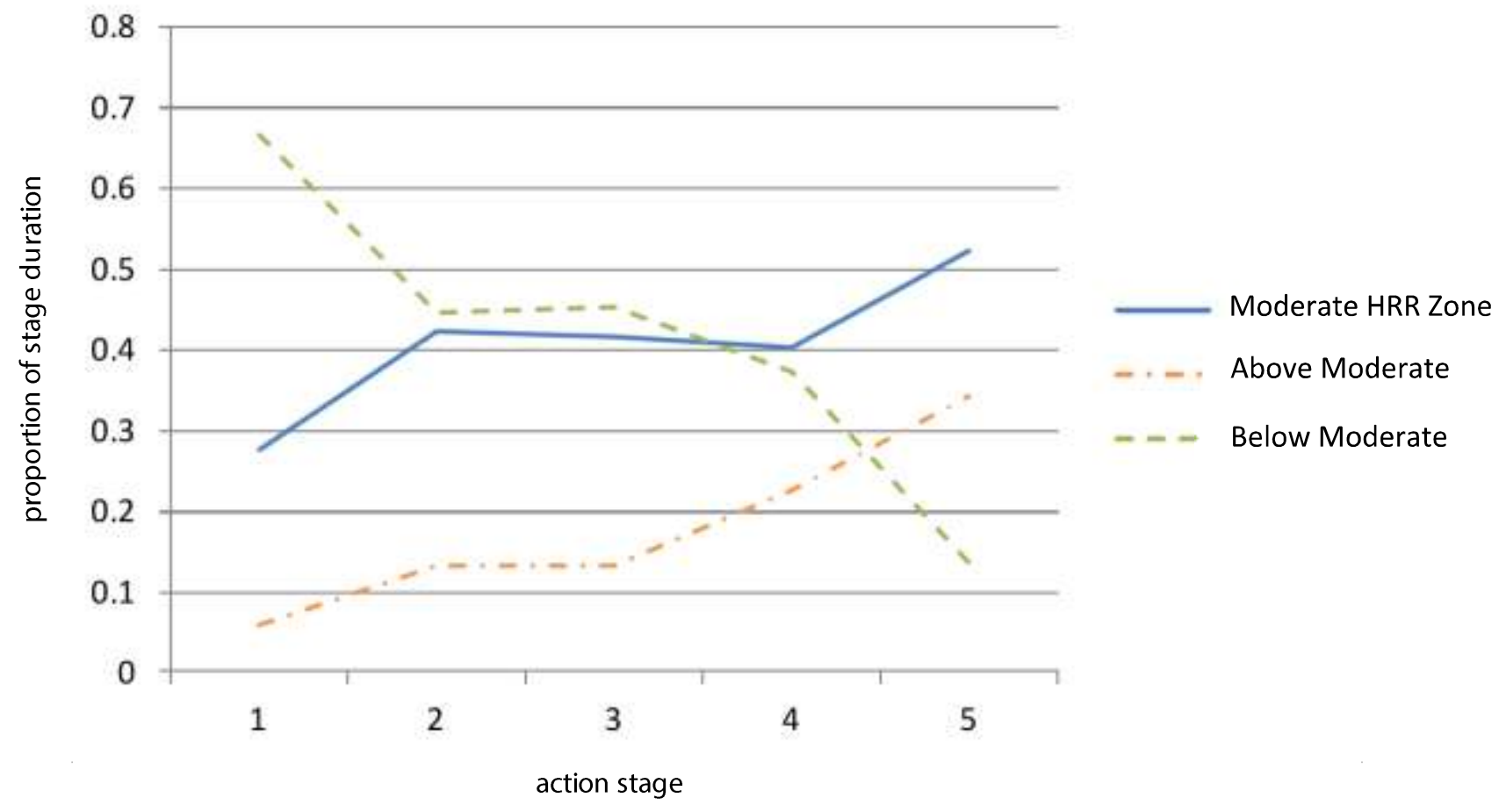

Figure 14 Average proportion of time spent in heart rate reserve intensity zones in each action stage, for all players overall, for both rounds averaged.

The two rounds are divided by a break that allows players to cool down for duration of their choosing. Each round sees this escalation in HRR. The second round tends to start a little warmer than the first, presumably due to carry-over from significant exertion in the first round.

The progression from cool to warm is not smooth, but falters at the midpoint. We suspect that the third action stage, featuring the Rigging Pull, is somewhat less demanding than the rest. This is not against our expectation, and it is not a bad thing in terms of exercise design, as a minor reprieve at the midpoint may help some players conserve stamina and complete the game. 
Section 3.3 describes our implementation of interval training exercise in a gamified form. As part of that description, Figure 11 illustrates the concept. The progression of high-intensity and low-intensity periods is described using an approximate sin wave. Since participants exhibited heart rate responses matching this rising-and-falling pattern, we believe the interval training concept was carried out successfully. Here, Figure 15 presents a typical example of heart rate results from one participant's session. The rolling average of the participant's heart rate is tracked over two rounds of play. Two approximate sin waves, similar to those in Figure 11, have been added to show the progression of the two rounds of the game. Each wave has five peaks, representing the five action stages of each round. The participant's heart rate reaction to the exertion demanded by the game approximately follows the conceptual outline, fulfilling an interval-based workout.

\subsubsection{Absence of Perceived Cognitive and Physical Obstacles}

Our confidence in responding to our research questions is bolstered by indications that there were no major difficulties for players interacting with the game. The game was comprehendible, the story was understandable, and the physical demand was reasonable. Highlights of the data are presented here; a detailed summary of survey results is available in Appendix E.

The story was rated as quite comprehendible (average 6.1, std dev 1.1) and the characters quite understandable (average 6.1, std dev 0.9).

The body poses required by the exercise were rated as fairly comfortable (average 5.5, std dev 1.1) and fairly understandable (average 5.3, std dev 1.1). Physical demand was rated moderately easy, not too challenging (average 5.4, std dev 1.3).

Overall, the game was perceived as quite straightforward and not confusing (average 6.5, std dev 0.7). The game was moderately easy to understand and play, rather than too challenging (average 5.08, std dev $1.2)$.

Based on these results, and on researcher observations during play sessions, we conclude that there were no outstanding issues with the game to interfere with the study. We find support that a moderate-intensity challenge is fulfilled. 


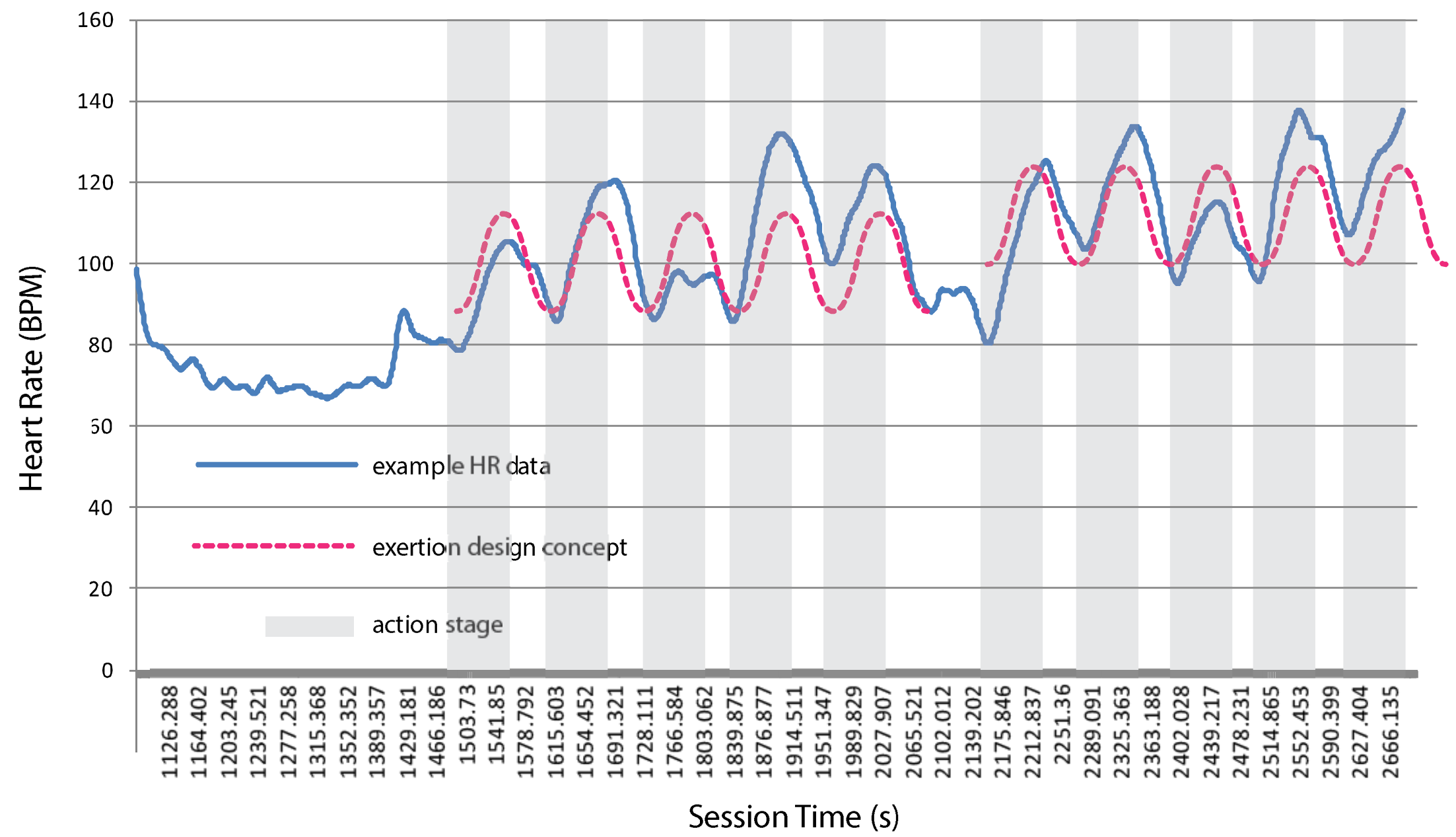

Figure 15 Typical HR result from a single participant illustrating interval training implementation 


\subsubsection{Correlations with Motion Gaming Experience}

Motion games are a super-category of exergames. The term refers to all video games played with some sort of bodily motion. This motion exceeds that of conventional gaming forms like controllers and keyboard, but it does not necessarily promote exercise that is meaningful to a player's fitness level. Exergames are motion games that do promote meaningful exercise. Results contain some disparities that may be associated with prior motion gaming experience. Implications for supporting and exploiting these attributes are noted in the Discussion.

\subsubsection{Disparity in Groups and Possibly Gender}

There was disparity between groups in motion gaming experience. The Normal group plays motion games much more in regular life (average 3.9 in a Likert range of 1 to 7 , std dev 2.3) than the Adapt group (average 2.1, std dev 1.2). The Normal group reports an awareness of many games that promote exercise, i.e. exergames (average 4.5, std dev 1.9) more than the Adapt group (average 4, std dev 1.9).

Disparity in motion gaming experience may be associated with a gender disparity. Males in both groups play more motion games in regular life (average 3.3) than females (average 2.7). There is a gender disparity in awareness of exergames (males more, average 4.7, females average 3.4) that is greater than the group disparity.

When asked about significant video game experience in regular life, there was not a great disparity between the Normal (average 5.2, std dev 2.4) and Adapt (5.1, std dev 2.2) groups. But males in both groups play video games more (average 5.8, std dev 1.9) than females (average 4, std dev 2.6). This isolates a gender disparity (rather than a group disparity) in video game experience, which would agree with a gender disparity in motion gaming experience.

The groups themselves have some gender disparity. The adapt group has 7 males and 5 females, and the Normal group has 9 males and 4 females. With the indication in mind that males, and therefore the Normal group, have more motion gaming experience, some differences in the results are identifiable that may be correlated to experience. 


\subsubsection{Desire for More Exergames}

We associate the disparity in motion gaming experience with other results. After playing, desire to play more exercise-based games was greater in the Normal group than the Adapt group (averages 5.6, std dev 0.9 , to 4.8 , std dev 1.4), and more consistent (variances of 0.9 to 2.0 ). Desire was greater in males than females overall (averages 5.4, std dev 1.4, to 5 , std $\operatorname{dev} 0.8$ ). That desire is correlated positively with reported motion gaming experience (correlation 0.22 ).

Therefore, we suspect that motion gaming experience is conducive to fostering a player's future desire for exergaming, after trying our story-driven approach to exergame design.

\subsubsection{Satisfaction with Game Feedback}

Both participant groups generally responded fairly similarly to the FSS. The flow components that saw the biggest differences between groups were ENJY (autotelic enjoyment, averages 4.9, std dev 0.9 for Adapt and 5.7, std dev 0.8for Normal in a Likert range of 1 to 7) and FDBK (clear feedback from game, averages 5.1, std dev 1.0 for Adapt and 6, std dev 0.7 for Normal). The responses for FDBK were also more consistent for the Normal group (variances 1.2 Adapt and 0.5 Normal).

We suspect that the Normal group, with more motion gaming experience, had less dependence on clear feedback. They were able to get into the game and comprehend the mechanics more easily because of their prior experiences. This may have impacted feelings of enjoyment during gameplay, given the results for ENJY.

This would agree with the results for our question (not part of the FSS) about whether the sound effects were good. As described in section 3.1.5, audio feedback is an essential part of our game feedback to the player. The Normal group thought the audio was better (average 5.7, std dev 1.0) than the Adapt group (average 4.6, std dev 1.2), with similar consistency (similar variance). This makes sense if the Normal group, more experienced motion gamers, has less reliance on clear feedback.

Alternatively, interpretation of the question "the sounds effects were good" may have been influenced by flow experiences. Audio feedback is provided by a light, pleasant noise for successful motions, and a more jarring noise for failed motions. The positive sound effect may have become white noise for players who were more focused or closer to optimal performance, at thus not noticed as much. This would lead to lower ratings, since there would be less conscious memory of the feedback. In this case, lower ratings for the audio by the Adapt group indicate stronger flow experience for that group, presumably due to the effect of adaptation for challenge management. 


\subsubsection{Story Investment}

The Normal group, who describe themselves as more experienced motion gamers, reported less investment in the story. We suspect that their experience made a story less critical for them to enjoy the experience, as they are relatively more accustomed to interacting with games using body movements

Table 3 presents notable responses supporting the notion that the story was less important for the Normal group, more experienced motion gamers. Differences in variances are approaching significance (insofar as applicable to Likert responses).

Table 3 Story Investment between Groups

\begin{tabular}{|l|l|l|l|l|l|l|l|l|}
\hline Question & \multicolumn{2}{|l|}{$\begin{array}{l}\text { A different story } \\
\text { would be preferable }\end{array}$} & $\begin{array}{l}\text { I cared about the } \\
\text { story, it was } \\
\text { compelling }\end{array}$ & $\begin{array}{l}\text { Story enhanced (or } \\
\text { did not detract } \\
\text { from) my } \\
\text { enjoyment }\end{array}$ & $\begin{array}{l}\text { I would be interested } \\
\text { in continuing the story } \\
\text { in a sequel game. }\end{array}$ \\
\hline & Adapt & Normal & Adapt & Normal & Adapt & Normal & Adapt & Normal \\
\hline $\begin{array}{l}\text { Mean } \\
+- \text { std dev }\end{array}$ & $\begin{array}{l}2.4 \\
+-1.3\end{array}$ & $\begin{array}{l}3.1 \\
+-1.6\end{array}$ & $\begin{array}{l}5.17 \\
+-1.3\end{array}$ & $\begin{array}{l}4.77 \\
+-1.5\end{array}$ & $\begin{array}{l}5.79 \\
+-0.7\end{array}$ & $\begin{array}{l}5.65 \\
+-1.2\end{array}$ & $\begin{array}{l}6.08 \\
+-0.9\end{array}$ & $\begin{array}{l}5.77 \\
+-1.7\end{array}$ \\
\hline Variance & 1.72 & 2.6 & 1.74 & 2.32 & 0.52 & 1.56 & 0.81 & 2.86 \\
\hline Median & 2 & 3 & 5 & 5 & 6 & 6 & 6 & 6 \\
\hline Mode & 4 & 4 & 4.5 & 5 & 6 & 6 & 7 & 7 \\
\hline Support & $\begin{array}{l}\text { Lower mean } \\
\text { indicates greater } \\
\text { affection for present } \\
\text { story. } \\
\text { (p-value 0.027) }\end{array}$ & $\begin{array}{l}\text { Higher mean } \\
\text { indicates greater } \\
\text { affection } \\
\text { (p-value 0.49) }\end{array}$ & $\begin{array}{l}\text { Lower variance } \\
\text { indicates more } \\
\text { consistent affection } \\
\text { (p-value 0.08) }\end{array}$ & $\begin{array}{l}\text { Lower variance } \\
\text { indicates more } \\
\text { consistent affection } \\
\text { (p-value 0.05) }\end{array}$ \\
\hline
\end{tabular}

Table shows responses in a Likert range of 1 to 7.

\subsubsection{Perception of Challenge}

We suspect that the more experienced group (Normal) perceived the physical challenge as less strenuous than the other group. Both groups indicated fairly strongly that they could have kept going in the exergame, even after the session was over, the Normal group more so (average 5.7, std dev 1.3 in a Likert range of 1 to 7) than the Adapt group (average 5, std dev 1.4).

Neither group overall felt that the physical demand became troublesome. The Normal group expressed this more strongly (average 2.8, std dev 2.0) than the Adapt group (average 3.5, std dev 1.5, where a response further below the midpoint of 4 is a stronger indication that the demand was not troublesome). However, the Normal group made this report with much less consistency (variance 4) than the Adapt group (variance 2.5), so this suspicion is questionable. 


\subsection{Discussion}

In this section we discuss implications of enhanced workout reliability, which has potential to help deliver exergaming benefit. We expound that applicability of interval-training with storytelling to higher intensity levels is not yet clear. Finally, there are some limitations in our game with which we must contend.

\subsubsection{Applying Reliability}

Our game targeted a moderate-intensity workout for players. According to heart rate data interpreted using ACSM standards, we achieved this for a considerable timeframe during play sessions, a timeframe that we consider appropriate for our story-driven interval training approach. For the group playing with adaptivity for challenge management, that achievement is suggested to have been more reliable, with a tighter grouping of results reflected by smaller variances.

This reliability, if confirmed by further study, could be exploited by allowing players to select their own level of exercise. As a home product, an exergame following the model set by our game could offer multiple settings. By incorporating adaptivity for challenge management, the designers could be confident that the player would experience the desired challenge.

Similarly, an expert person or algorithm could be enabled to prescribe a level of exercise for an individual player. A game that incorporated more medical or physiotherapeutic insight than the present study could leverage adaptivity by targeting a certain setting, either by being set manually by a health professional or being guided by a procedural model designed by health professionals. That would be towards fulfilling a need for health-enabled exergames [17].

\subsubsection{Graduating to Higher Intensity Levels}

The game is designed to target a moderate-intensity workout, with or without adaptivity for challenge management. Our most direct measure of success with regards to invoking exercise is the proportion of time spent in the heart-rate response (HRR) zone indicating moderate exertion. While designing the study, targeting a moderate intensity was a design decision meant to set a modest, achievable goal for our untested exergame format. However, there are two reasons we may wish to graduate the game to a higher intensity level. 
The first reason is that our choice to target a moderate intensity may have limited the clarity of our results. Participants had a variety of fitness levels and heart performances. Some people tended to overshoot our HRR target zone, exhibiting heart rate higher than the target during exertion, and some tended to undershoot. Adaptation seems to have gone some way to addressing this, hence the signs of increased consistency for the Adapt group. Our analysis may have been clearer if our target was a high-intensity workout, which players are less likely to overshoot.

With overshooting the target HRR zone being less of a concern, it would be a simpler matter to adapt the level of challenge in order to push players into higher and higher HRR response, instead of primarily trying to get it "just right". Results between the two participant groups might be more clear-cut, since the Normal group might be less likely to achieve high-intensity workout results on average to the same degree as the Adapt group.

The second reason a higher intensity is desirable is simply to advance the flexibility of the game. In the long term, a player may not wish to stay at the same workout intensity. In order to fulfill increasing fitness goals, the level of challenge must be escalated over time.

Results indicate at least some time was spent by participants in the high-intensity HRR zone, meaning that the game is already able to push players into the high zone. However, for future iterations of the game to be redesigned thoroughly in order to graduate to higher intensity levels, some considerations are discussed in section 5.1.1.

\subsubsection{Viability for Higher Intensity Levels}

Our game design exploits a similarity between narrative progression in games and the periodic intensity of interval training discussed in section 3.3.1. This served the design by affording a timeframe for telling a story in which the exercise was contextualized. Our observations support this approach for our selected purpose of offering a moderate-intensity workout.

Similar to conventional high-intensity interval training (HIIT) routines, our game demands significant exertion on a periodic basis. However, there are considerations for expanding our story-driven approach which introduce differences between our game and HIIT. Those differences may challenge the viability of our design at higher intensity levels. 
HIIT typically involves repetition of a single exercise motion over multiple sets. Our game, on the other hand, involves sets of multiple different exercises. The interval training offered by our game could be thought of as "impure" in this regard - instead of targeting one muscle group or movement type, we move rapidly between types.

This variety of moves is necessary for our style of contextualization in the story; however it may not be essentially necessary for story-driven exergames generally. For instance, it is not unheard of for racing games to tell stories; Need for Speed: The Run [77], directed by Michael Bay, is an example. A racingbased exergame which uses a cycling mechanic at all times might follow that game's example of compelling narrative told mostly through a single kind of racing gameplay. A narrative campaign could be developed for Heart Burn [49], for example.

Another difference separates our approach from typical HIIT. Interval training is often described in terms of a "time-on, time-off" ratio of the duration of exercise periods against the duration of rest periods. A ratio of 2:1 is typical [54] for workouts that are high-intensity overall, whereas our moderate-intensity workout offers a ratio of about 1:1 for design reasons detailed in section 3.3.2.

Graduating to a higher-intensity might require increasing the time-on, time-off ratio. Depending on the resulting durations, this requires some consideration. If time-on is increased, care must be taken to not overwhelm the endurance of players. Adaptivity for challenge management may be able to help in this regard. If time-off is decreased, this puts pressure on the time allotment for storytelling. For future games, designers could consider how to advance the plot during the exercise in addition to doing so between sets, thereby alleviating time constraints. Whether storytelling in the same moment as exergaming interaction is conducive to player engagement is open to investigation.

\subsubsection{Improving Feedback Clarity and Appeal}

As outlined in section 3.1.5, our game incorporates several elements of feedback to the player, both visual and audio. During play sessions, we collected some encouraging positive comments, particularly about the appropriateness and helpfulness of the sound effects. Less often, there were some negative comments from players who were less successful about the clarity of feedback or the responsiveness of the game overall. This might be due to player performance, or from technical shortcomings. While being mindful of the limitations of our game, we find these comments encouraging as well, in a sense: Players are disturbed if they feel they aren't "doing it right" (whether it is indeed their doing or an intermittent fault in the game). This reflects a desire for mastery and success, and hence investment in the game experience. 
Although the feedback elements were generally well-received, some comments indicated that the visuals could be even simpler than they are currently. In the current version of the game, the player has three places to look during play: at the avatar, at the non-player characters providing an example to follow, and at the combo counter indicating the consistency of player performance. Reducing the number of places to look by subtracting from or combining those visual elements might increase the accessibility of the game. Visual user interface factors in exergaming are open to investigation.

The mixed results make it clear that the feedback elements of our game could stand to be improved. Particularly, section 4.1.2.3 implies a demand for better feedback to support less experienced motion gamers. For audio, better sound effects could be selected, perhaps a variety of randomized effects to mitigate annoying repetition. For visual feedback, we have admired the presentation style of Ubisoft's Your Shape: Fitness Evolved [78], which uses real-time stylized visualization of the entire body. Implementing a similar visualization might be a good next step for future versions of our game. This would be a clear advancement over the current method of using player input to decide which animation to play next. 


\section{Conclusion}

Exergames have the potential to leverage the attractiveness of gaming in order to deliver an effective fitness solution. To explore the ways in which they may be designed to do this, we have presented an original exergame in which players perform pose-based input through wearable sensors.

The game is theoretically rooted in psychological flow in order to bridge experiential aspects of gaming and exercise. By examining a broad variety of flow research, guidelines were identified which informed our design. Service-based gamification [22] has been employed in order to integrate exercise into a gaming experience.

Adaptivity for challenge management has been implemented to help fulfill the objectives of the dual-flow model [45]. Interval training has been implemented in conjunction with an expandable story-driven approach to game content.

The flow experiences of players have been evaluated, as well as heart-rate based measurements of exercise. The workout received by players is suggested to have been more reliable when their play was moderated by adaptation. The story-driven approach was seen as compelling, and generated interest in continuing to play exergames.

Criticism of the game's feedback fidelity has been heeded. The potential of adaptivity to help deliver exergaming benefit has been suggested as increased workout reliability. The outlook for story-driven, interval training style exergames is positive according to player responses, but it is subject to further consideration when graduating the gameplay to higher intensity levels. A player's degree of prior experience may also be a factor in the value of story-driven exergaming.

\section{$5.1 \quad$ Future Considerations}

The discussion of results has been based in large part on what could have been better or how the game could be expanded or adjusted to new contexts or objectives. By describing our results in a positive, hypothetical light, we hope to foster further development of our concept. There remain some distinct considerations for future iterations of our game, and other exergames. 


\subsubsection{Scalability of Story-Driven Approach}

The viability a story-driven approach to exergame design has been suggested by results. Results showed that players were interested in the story, understood it and became invested in it. We have detected no way in which the story was a hindrance to the attractiveness or effectiveness of the game.

A logical next step for our game is to increase the depth of interaction between the player's input and the results in the world of the game. Several participants commented that they felt an expectation for the story to be altered in some way depending on their performance. One way to implement this, for example, would be that each level has a pass or failure condition that must be fulfilled with a certain score or other metric. This would be similar to many linear video games where each major challenge must be met in succession. With this feature in our game, a player might fail the sword fighting level and be defeated, and have to retry it in order to progress.

The issue with approaching increased interaction depth in this linear manner is the sustainability of interest in meeting the challenge using exercise moves. While a player can choose to persist against a major challenge in a conventional video game many times, physical fatigue is a limiting factor in exergames. If the player is unable to progress steadily enough, the pacing of the story may be disrupted, and the player's interest in it as well.

Another approach to increasing interaction depth that would avoid this linear problem is branching paths. If the player was defeated in that swordfight, the story could continue in a whole new direction. However, this design approach faces the same issue as all choose-your-own-adventure stories: content demand. Branching paths mean there are many possible courses for the player's journey, yet each player might only see one path among those many, unless they replay the game. This is an inefficient content presentation strategy, demanding a lot of content that any given player might never see. There is research in procedurally generating stories and quests that might allow designers to avoid this inefficiency by letting the game create more content as needed automatically $[31,60,76]$. 
Alternatively to procedural story generation, a solution may lie in exploiting the experiential qualities of experienced motion gamers, relative to inexperienced ones. We found reason to suspect that more experienced motion gamers found the presence of a story less critical to enjoying the game. This could be applied to future initiatives for fitness benefit through gaming. Players could be introduced to motion gaming form factors and experiences using games that are known to be entertaining and which deliver deep interaction, but which are not necessarily focused on fitness. Later, those experiences could provide a bridge into more exercise-focused games. At that point, according to our suspicion, the demand for story interaction would be reduced, and thus the demand for content on game creators is reduced. This progression of "easing players in" to exergaming could be an efficient way to deliver exercise benefit while supporting player demand for interaction with high-quality game content at the right time in the player's progression.

\subsubsection{Dual-Axis Adaptation}

The dual-flow model is centred on the challenge encountered by exergame players. It is described in terms of the two components of an exergaming challenge: game challenge influencing attractiveness, and physical challenge influencing effectiveness. These aspects ultimately culminate in a single, integrated player experience. The approach we have taken is to address both aspects at once. By motivating simple self-competition with a combo counter, and relying on investment in a story, we attempt to create an experience that fulfills dual flow using a single, unified game mechanic.

In future, it might be compelling to unpack this approach with a more nuanced mechanic, one which addresses each aspect of challenge more separately and distinctly. In terms of adaptation, such a mechanic would involve more axes of adjustment. While our game has a single axis, the pace of the motions, a game which addresses the dual-flow aspects of challenge separately would probably have a dual-axis mechanic. Using adaptation, this would afford game designers and researchers a finer degree of control over the player experience, potentially increasing its optimality.

In a dual-axis exergame mechanic, one axis would focus particularly on managing physical challenge, and another would focus particularly on managing game challenge. Since the result is still a single unified player experience, the focus of each axis is not entirely exclusive, and the effect of one would probably bleed into the other's experiential influence. Whether this bleeding effect is manageable is an open question that will depend of the particular application. 
For example, an exergame could employ pace (the rate at which moves are regularly performed) as one axis, and complexity (the variety of different moves needed to be performed) as another axis. Sea Monkeys in its current form to employs pace. The game could be expanded with a pose-matching or gesture-matching mechanic, contextualized in the story as a training session for sword duels, in order to employ complexity. In this mechanic, the pace of motion is the physical axis and the complexity of combinations of moves is the game axis. It would be akin to an improvisational dancing game, requiring repeated demonstration of a procedurally generated move sequence by a trainer character.

A dual-axis adaptive exergame would also require a more complex model of the player. In section 3.4.1 we considered adaptation to be composed of two parts, and evaluation and an adjustment axis, which feed into each other to complete a feedback loop. In addition to the adjustment axes just described, a dual-axis mechanic would require evaluation elements to close two feedback loops. As evaluation for the pace axis, our game monitors and models the player's heart and treats heart performance as equivalent to exercise. As evaluation for the complexity axis, the dynamic sword training mechanic could track the rate and accuracy of move completion.

A dual-axis adaptation mechanic might create a more reliably optimized player experience than a singleaxis one. The main design challenge would be effectively separating the two aspects of challenge, game and physical. That challenge would be one of mitigation rather than an obstacle to be totally overcome, because in the end, the result is one unified experience for the player.

As a bridge to dual-axis adaptation, future exergaming studies could focus on adaptation axes other than pace, to examine their effects in isolation. In this study, our results suggest some success in improving physical workout by adjusting pace. However, our flow-state results did not directly indicate an effect of pace adjustment on flow experience. Studying an alternative adaptation axis, such as complexity or accuracy, may yield clearer indications. 


\section{References}

1. Adams, M.A., Marshall, S.J., Dillon, L., et al. A theory-based framework for evaluating exergames as persuasive technology. Proceedings of the 4th International Conference on Persuasive Technology - Persuasive '09, ACM Press (2009), 1.

2. Baradoy, G. A Physiological Feedback Controlled Exercise Video Game. 2012. http://theses.ucalgary.ca/handle/11023/311.

3. Bast, M. The gamification of weight loss. TED Blog. http://blog.ted.com/2014/01/02/thegamification-of-weight-loss/.

4. Beard, K.S. and Hoy, W.K. The Nature, Meaning, and Measure of Teacher Flow in Elementary Schools: A Test of Rival Hypotheses. Educational Administration Quarterly 46, 3 (2010), 426-458.

5. Beume, N., Danielsiek, H., Eichhorn, C., et al. Measuring flow as concept for detecting game fun in the Pac-Man game. 2008 IEEE Congress on Evolutionary Computation (IEEE World Congress on Computational Intelligence), IEEE (2008), 3448-3455.

6. Bogost, I. Gamification is bullshit. 2011.www.bogost.com/blog/gamification_is_bullshit.shtml.

7. Camarda, S.R. de A., Tebexreni, A.S., Páfaro, C.N., et al. Comparison of maximal heart rate using the prediction equations proposed by Karvonen and Tanaka. Arquivos brasileiros de cardiologia 91, 5 (2008), 311-4.

8. Chen, H., Wigand, R.T., and Nilan, M.S. Optimal experience of Web activities. Computers in Human Behavior 15, 5 (1999), 585-608.

9. Chou, T.-J. and Ting, C.-C. The role of flow experience in cyber-game addiction. Cyberpsychology \& behavior : the impact of the Internet, multimedia and virtual reality on behavior and society 6 , 6 (2003), 663-75.

10. Christison, A. and Khan, H.A. Exergaming for health: a community-based pediatric weight management program using active video gaming. Clinical pediatrics 51, 4 (2012), 382-8.

11. Csikszentmihalyi, M. and LeFevre, J. Optimal experience in work and leisure. Journal of Personality and Social Psychology 56, 5 (1989), 815-822.

12. Csikszentmihalyi, M. Flow: The Psychology of Optimal Experience. HarperCollins, 2008.

13. Epstein, Z. Microsoft says Xbox 360 sales have surpassed 76 million units, Kinect sales top 24 million. BGR. http://bgr.com/2013/02/12/microsoft-xbox-360-sales-2013-325481/.

14. Gackenbach, J.I. The Relationship Between Perceptions of Video Game Flow and Structure. Loading... 2, 3 (2008). 
15. Gellish, R.L., Goslin, B.R., Olson, R.E., McDonald, A., Russi, G.D., and Moudgil, V.K. Longitudinal modeling of the relationship between age and maximal heart rate. Medicine and Science in Sports and Exercise 39, 5 (2007), 822-9.

16. Ghani, J.A. and Deshpande, S.P. Task Characteristics and the Experience of Optimal Flow in Human-Computer Interaction. The Journal of Psychology 128, 4 (1994), 381-391.

17. Göbel, S., Hardy, S., Wendel, V., Mehm, F., and Steinmetz, R. Serious games for health. Proceedings of the international conference on Multimedia-MM '10, ACM Press (2010), 1663.

18. Goldfield, G.S., Kalakanis, L.E., Ernst, M.M., and Epstein, L.H. Open-loop feedback to increase physical activity in obese children. International journal of obesity and related metabolic disorders : journal of the International Association for the Study of Obesity 24, 7 (2000), 888-92.

19. Graves, L., Stratton, G., Ridgers, N.D., and Cable, N.T. Comparison of energy expenditure in adolescents when playing new generation and sedentary computer games: cross sectional study. BMJ (Clinical research ed.) 335, 7633 (2007), 1282-4.

20. Gulati, M., Shaw, L.J., Thisted, R.A., Black, H.R., Bairey Merz, C.N., and Arnsdorf, M.F. Heart rate response to exercise stress testing in asymptomatic women: the st. James women take heart project. Circulation 122, 2 (2010), 130-7.

21. Hsu, C.-L. and Lu, H.-P. Why do people play on-line games? An extended TAM with social influences and flow experience. Information \& Management 41, 7 (2004), 853-868.

22. Huotari, K. and Hamari, J. Defining gamification. Proceeding of the 16th International Academic MindTrek Conference on - MindTrek'12, ACM Press (2012), 17.

23. Inal, Y. and Cagiltay, K. Flow experiences of children in an interactive social game environment. British Journal of Educational Technology 38, 3 (2007), 455-464.

24. Inbar, O., Oren, A., Scheinowitz, M., Rotstein, A., Dlin, R., and Casaburi, R. Normal cardiopulmonary responses during incremental exercise in 20- to 70-yr-old men. Medicine and science in sports and exercise 26, 5 (1994), 538-46.

25. Jackson, S.A. and Eklund, R.C. Assessing flow in physical activity: The Flow State Scale-2 and Dispositional Flow Scale-2. Journal of Sport \& Exercise Psychology 24, 2 (2002), 133-150.

26. Jackson, S.A. and Marsh, H. Development and validation of a scale to measure optimal experience: The Flow State Scale. Journal of Sport \& Exercise Psychology 18, 1 (1996), 17-35.

27. Jegers, K. Pervasive game flow. Computers in Entertainment 5, 1 (2007), 9.

28. Kolata, G. 'Maximum' Heart Rate Theory Is Challenged. The New York Times, 2001. http://www.nytimes.com/2001/04/24/health/maximum-heart-rate-theory-is-challenged.html. 
29. Laursen, P.B. and Jenkins, D.G. The Scientific Basis for High-Intensity Interval Training: Optimising Training Programmes and Maximising Performance in Highly Trained Endurance Athletes. Sports Medicine 32, 1 (2002), 53-73.

30. Lee, I. and Kwon, H.-J. Relations among Flow, Information Processing Strategies, and Performance in a Computer-based Simulation Game. Proceedings of World Conference on Educational Multimedia, Hypermedia and Telecommunications 2005, AACE (2005), 986-992.

31. Li, B. and Riedl, M.O. An offline planning approach to game plotline adaptation. Proceedings of the Sixth AAAl Conference on Artificial Intelligence and Interactive Digital Entertainment, (2010).

32. Lin, J.J., Mamykina, L., Lindtner, S., Delajoux, G., and Strub, H.B. Fish'n'Steps: encouraging physical activity with an interactive computer game. UbiComp'06 Proceedings of the 8th international conference on Ubiquitous Computing, (2006), 261-278.

33. Luke, R.C. Oxygen cost and heart rate response during interactive whole body video gaming. 2005.

34. Macvean, A.P. Developing adaptive exergames for adolescent children. Proceedings of the 11th International Conference on Interaction Design and Children - IDC '12, ACM Press (2012), 339.

35. Mandryk, R.L., Inkpen, K.M., and Calvert, T.W. Using psychophysiological techniques to measure user experience with entertainment technologies. Behaviour \& Information Technology 25, 2 (2006), 141-158.

36. McLellan, H. Experience Design. CyberPsychology \& Behavior 3, 1 (2000), 59-69.

37. Nickel, A. Designing better exergames: Application of flow concepts and the FITT principle to full body exertion video games and flexible challenge systems. 2013, 187. http://search.proquest.com/docview/1496764878/abstract?accountid=9894.

38. Pagoto, S., Schneider, K., Jojic, M., DeBiasse, M., and Mann, D. Evidence-based strategies in weight-loss mobile apps. American journal of preventive medicine 45, 5 (2013), 576-82.

39. Parfitt, B. JAPAN: Wii Fit sells one million. MCVUK.com. http://www.mcvuk.com/news/read/japan-wii-fit-sells-one-million.

40. Payne, B.R., Jackson, J.J., Noh, S.R., and Stine-Morrow, E.A.L. In the zone: flow state and cognition in older adults. Psychology and aging 26, 3 (2011), 738-43.

41. Quinn, R.W. Flow in Knowledge Work: High Performance Experience in the Design of National Security Technology. Administrative Science Quarterly 50, 4 (2005), 610-641.

42. Robergs, R.A. and Landwehr, R. The surprising history of the "HRmax=220-age" equation. Official Journal of The American Society of Exercise Physiologists 5, 2 (2002), 1-10. 
43. Rogatko, T.P. The Influence of Flow on Positive Affect in College Students. Journal of Happiness Studies 10, 2 (2007), 133-148.

44. Saelens, B.E. and Epstein, L.H. Behavioral engineering of activity choice in obese children. International journal of obesity and related metabolic disorders : journal of the International Association for the Study of Obesity 22, 3 (1998), 275-7.

45. Sinclair, J., Hingston, P., and Masek, M. Considerations for the design of exergames. Proceedings of the 5th international conference on Computer graphics and interactive techniques in Australia and Southeast Asia - GRAPHITE '07, ACM Press (2007), 289.

46. Skadberg, Y.X. and Kimmel, J.R. Visitors' flow experience while browsing a Web site: its measurement, contributing factors and consequences. Computers in Human Behavior 20, 3 (2004), 403-422.

47. Smith, A., Lewis, C., and Hullett, K. An inclusive taxonomy of player modeling. University of California, Santa Cruz, (2011).

48. Smith, A.M., Lewis, C., Hullet, K., and Sullivan, A. An inclusive view of player modeling. Proceedings of the 6th International Conference on Foundations of Digital Games - FDG '11, ACM Press (2011), 301-303.

49. Stach, T., Graham, T.C.N., Yim, J., and Rhodes, R.E. Heart rate control of exercise video games. Proceedings of Graphics Interface 2009, Canadian Information Processing Society (2009), 125132.

50. Staiano, A.E. and Calvert, S.L. Exergames for Physical Education Courses: Physical, Social, and Cognitive Benefits. Child development perspectives 5, 2 (2011), 93-98.

51. Stavrou, N.A. and Zervas, Y. Confirmatory factor analysis of the flow state scale in sports. International Journal of Sport and Exercise Psychology 2, 2 (2004), 161-181.

52. Sweetser, P., Johnson, D., Ozdowska, A., and Wyeth, P. GameFlow heuristics for designing and evaluating real-time strategy games. Proceedings of The 8th Australasian Conference on Interactive Entertainment Playing the System - IE '12, ACM Press (2012), 1-10.

53. Sweetser, P. and Wyeth, P. GameFlow. Computers in Entertainment 3, 3 (2005), 3.

54. Talanian, J.L., Galloway, S.D.R., Heigenhauser, G.J.F., Bonen, A., and Spriet, L.L. Two weeks of high-intensity aerobic interval training increases the capacity for fat oxidation during exercise in women. Journal of Applied Physiology 102, 4 (2007), 1439-1447.

55. Tan, B., Aziz, A.R., Chua, K., and Teh, K.C. Aerobic demands of the dance simulation game. International journal of sports medicine 23, 2 (2002), 125-9. 
56. Tanaka, H., Monahan, K.D., and Seals, D.R. Age-predicted maximal heart rate revisited. Journal of the American College of Cardiology 37, 1 (2001), 153-156.

57. Tenenbaum, G. and Fogarty, G.J. The Flow Experience: A Rasch Analysis of Jackson's Flow State Scale. Journal of Outcome Measurement 3, 3 (1999), 278-294.

58. Thames, R.C. Dungeon Flow: Player Experiences of Flow in World of Warcraft. 2010. http://wakespace.lib.wfu.edu/jspui/bitstream/10339/30415/1/Thames_wfu_0248M_10019.pdf.

59. Thompson, W.R., Gordon, N.F., and Pescatello, L.S., eds. ACSM's Guidelines for Exercise Testing and Prescription. Lippincott Williams \& Wilkins, Philadelphia, 2010.

60. Thue, D., Bulitko, V., and Spetch, M. Interactive storytelling: A player modelling approach. Intelligence and Interactive, July (2007), 43-48.

61. Trost, S.G., Sundal, D., Foster, G.D., Lent, M.R., and Vojta, D. Effects of a Pediatric Weight Management Program With and Without Active Video Games: A Randomized Trial. JAMA pediatrics, (2014).

62. Vlachopoulos, S.P., Karageorghis, C.I., and Terry, P.C. Hierarchical confirmatory factor analysis of the Flow State Scale in exercise. Journal of sports sciences 18, 10 (2000), 815-23.

63. Wang, L.-C. and Chen, M.-P. The effects of game strategy and preference-matching on flow experience and programming performance in game-based learning. Innovations in Education and Teaching International 47, 1 (2010), 39-52.

64. Whitehead, A., Crampton, N., Fox, K., and Johnston, H. Sensor networks as video game input devices. Proceedings of the 2007 conference on Future Play - Future Play'07, ACM Press (2007), 38.

65. Whitehead, A., Johnston, H., Fox, K., Crampton, N., and Tuen, J. Homogeneous accelerometerbased sensor networks for game interaction. Computers in Entertainment 9, 1 (2011), 1-18.

66. Whitehead, A., Johnston, H., Nixon, N., and Welch, J. Exergame effectiveness. Proceedings of the 5th ACM SIGGRAPH Symposium on Video Games - Sandbox '10, ACM Press (2010), 55-62.

67. Wohlfart, B. and Farazdaghi, G.R. Reference values for the physical work capacity on a bicycle ergometer for men - a comparison with a previous study on women. Clinical Physiology and Functional Imaging 23, 3 (2003), 166-170.

68. Xin Ding, D., Hu, P.J.-H., Verma, R., and Wardell, D.G. The Impact of Service System Design and Flow Experience on Customer Satisfaction in Online Financial Services. Journal of Service Research 13, 1 (2009), 96-110.

69. Yannakakis, G. and Odense, M. Comparative fun analysis in the innovative playware game platform. Proceedings of the 1st World Conference for Fun 'n Games, (2006), 1-8. 
70. Yannakakis, G.N. and Togelius, J. Experience-Driven Procedural Content Generation. IEEE Transactions on Affective Computing 2, 3 (2011), 147-161.

71. Yim, J. and Graham, T.C.N. Using games to increase exercise motivation. Proceedings of the 2007 conference on Future Play - Future Play '07, ACM Press (2007), 166.

72. Yin-Poole, W. PlayStation 3 hits 70 million units shipped worldwide mark six years after launch. Eurogamer. http://www.eurogamer.net/articles/2012-11-16-playstation-3-hits-70-million-unitsshipped-worldwide-mark-six-years-after-launch.

73. Zagal, J., Björk, S., and Lewis, C. Dark Patterns in the Design of Games. Foundations of Digtal Games, (2013), 8.

74. Zephyr Technology Corporation. Validity of BioHarness ${ }^{\mathrm{TM}}$ Heart Rate vs 3-lead ECG.

ZephyrAnywhere white papers, 2008. http://www.zephyr-

technology.com/store/images/stories/pdf/whitepaper_heartratevalidation.pdf.

75. Zephyr Technology Corporation. HxM BT - Wireless Bluetooth Heart Rate Monitor for Android \& Windows Phone 8. ZephyrAnywhere products page, 2012.

http://www.zephyranywhere.com/products/hxm-bluetooth-heart-rate-monitor/.

76. Zook, A., Lee-Urban, S., Drinkwater, M., and Riedl, M.O. Skill-based Mission Generation: A Datadriven Temporal Player Modeling Approach. (2012).

77. Need for Speed: The Run for Xbox 360 Reviews. Metacritic, 2011.

http://www.metacritic.com/game/xbox-360/need-for-speed-the-run.

78. Your Shape: Fitness Evolved - Sales. VGChartz, 2014.

http://www.vgchartz.com/game/45626/your-shape-fitness-evolved/. 


\section{Appendix A Comparison of Formulas for Maximum Heart Rate}

The first column lists a set of ages. The following columns list the result of each formula based on the age.

\begin{tabular}{|c|c|c|c|c|c|c|c|c|c|}
\hline Age & $\begin{array}{l}\text { Gellish A } \\
\text { (selected) }\end{array}$ & Gellish B & Gellish C & $\begin{array}{l}\text { Gulati } \\
\text { (women) }\end{array}$ & $\begin{array}{l}\text { Wohlfart } \\
\text { (men) }\end{array}$ & $\begin{array}{l}\text { Wohlfart } \\
\text { (women) }\end{array}$ & $\begin{array}{l}\text { Inbar via } \\
\text { Robergs }\end{array}$ & Tanaka & $\begin{array}{l}\text { Haskell } \\
\& \text { Fox }\end{array}$ \\
\hline 20 & 189.2 & 179 & 193 & 188.4 & 191.8 & 186.7 & 192.1 & 194 & 200 \\
\hline 25 & 187.6 & 180.8 & 189.5 & 184 & 189.8 & 185.8 & 188.675 & 190.5 & 195 \\
\hline 30 & 185.7 & 181.6 & 186 & 179.6 & 187.5 & 184.7 & 185.25 & 187 & 190 \\
\hline 35 & 183.4 & 181.6 & 182.5 & 175.2 & 184.9 & 183.3 & 181.825 & 183.5 & 185 \\
\hline 40 & 180.8 & 180.6 & 179 & 170.8 & 181.9 & 181.7 & 178.4 & 180 & 180 \\
\hline 45 & 177.8 & 178.8 & 175.5 & 166.4 & 178.5 & 179.6 & 174.975 & 176.5 & 175 \\
\hline 50 & 174.5 & 176 & 172 & 162 & 174.6 & 177.1 & 171.55 & 173 & 170 \\
\hline 55 & 170.8 & 172.4 & 168.5 & 157.6 & 170.2 & 174.1 & 168.125 & 169.5 & 165 \\
\hline 60 & 166.8 & 167.8 & 165 & 153.2 & 165.4 & 170.4 & 164.7 & 166 & 160 \\
\hline 65 & 162.4 & 162.4 & 161.5 & 148.8 & 160 & 166 & 161.275 & 162.5 & 155 \\
\hline 70 & 157.7 & 156 & 158 & 144.4 & 154 & 160.8 & 157.85 & 159 & 150 \\
\hline 75 & 152.6 & 148.8 & 154.5 & 140 & 147.6 & 154.7 & 154.425 & 155.5 & 145 \\
\hline 80 & 147.2 & 140.6 & 151 & 135.6 & 140.6 & 147.7 & 151 & 152 & 140 \\
\hline 85 & 141.4 & 131.6 & 147.5 & 131.2 & 133.2 & 139.8 & 147.575 & 148.5 & 135 \\
\hline 90 & 135.3 & 121.6 & 144 & 126.8 & 125.4 & 130.9 & 144.15 & 145 & 130 \\
\hline
\end{tabular}




\section{Appendix B Survey Instrument}

Following is the survey instrument presented to each participant of the study. When presented to participants, the Likert questions appear in a randomized order. Many questions are reversed versions of other questions for verification.

Personal Data

Participant ID

Age

Gender

Health conditions related directly to heart

On a scale of $1-7$ ( 1 = strongly disagree, 7 = strongly agree $)$, please indicate your level of agreement with each of the following statements.

\begin{tabular}{|l|l|l|l|l|l|l|}
\hline 1 & 2 & 3 & 4 & 5 & 6 & 7 \\
\hline $\begin{array}{l}\text { strongly } \\
\text { disagree }\end{array}$ & disagree & $\begin{array}{l}\text { somewhat } \\
\text { disagree }\end{array}$ & $\begin{array}{l}\text { neither } \\
\text { agree or } \\
\text { disagree }\end{array}$ & $\begin{array}{l}\text { somewhat } \\
\text { agree }\end{array}$ & agree & $\begin{array}{l}\text { strongly } \\
\text { agree }\end{array}$ \\
\hline
\end{tabular}

\section{Past Experience Questions:}

In regular life, I get a significant amount of exercise (through any means).

In the past, I have played a significant amount of video games.

In the past, I have played a significant amount of video games controlled by body motion.

I am aware of a lot of video games that promote physical exertion and exercise.

\section{General Questions:}

The game was fun.

The game was boring.

The game was entertaining.

The game was straightforward.

The game was confusing.

After playing the game, I want to continue playing exercise games.

\section{Game Quality Questions:}

The graphics and animations were good.

The sound effects were good.

\section{Game Learning Questions:}

I understood how to do the poses.

Trying to do the poses was confusing at times.

The tutorial images were helpful to learn the poses.

The game needs a better way to tell me what poses to do.

\section{Story Involvement Questions:}

I understood the characters.

I did not recognize the characters.

I followed the events of the story.

The story was confusing. 
I cared about the events of the story.

The story was not compelling.

I would be interested in continuing the story in a sequel game.

A totally different story would be preferable to play.

My enjoyment was enhanced by the story.

The story detracted from my enjoyment of the game.

The story helped me exercise.

The story was distracting.

After playing the game, I want more entertainment involving pirates.

\section{Exercise Questions:}

Playing the game was easy.

The game was challenging to play.

The body poses were awkward.

It was comfortable to do the body poses.

I easily kept up with the physical demand of the game.

The game's physical demand was too much at times.

The game stopped at the right time.

Physically, I could have kept playing even after the game was over.

Playing the game was fine for a while, but then the physical demand became troublesome.

The Flow State Scale [26] (originally a likert 1-5) ([57] lists relation to components)

1. I was challenged, but I believed my skills would allow me to meet the challenge.

2. I made the correct movements without thinking about trying to do so.

3. While playing, I knew clearly what I wanted to do.

4. It was really clear to me that I was doing well.

5. My attention was focused entirely on what I was doing.

6. I felt in total control of what I was doing.

7. I was not concerned with what others may have been thinking of me while playing.

8. Time seemed to alter (either slowed down or speeded up) while playing.

9. I really enjoyed the experience.

10. My abilities matched the high challenge of the situation.

11. While playing, it felt like things were just happening automatically.

12. I had a strong sense of what I wanted to do while playing.

13. I was aware of how well I was performing.

14. It was no effort to keep my mind on what was happening.

15. I felt like I could control what I was doing.

16. I was not worried about my performance during the event.

17. The way time passed seemed to be different from normal while playing.

18. I loved the feeling of that session and want to capture it again.

19. I felt I was competent enough to meet the high demands of the situation.

20. While playing, I performed automatically.

21 . I knew what I wanted to achieve.

22. I had a good idea while I was performing about how well I was doing.

23. I had total concentration.

24. I had a feeling of total control.

25. While playing, I was not concerned with how I was presenting myself.

26. It felt like time stopped while I played.

27. The experience left me feeling great.

28. The challenge and my skills were at an equally high level. 
29. I did things spontaneously and automatically without having to think.

30. My goals were clearly defined.

31 . I could tell by the way I was performing how well I was doing.

32. I was completely focused on the task at hand.

33. I felt in total control of my body.

34. I was not worried about what others may have been thinking of me.

35. At times, it almost seemed like things were happening in slow motion.

36. I found the experience extremely rewarding. 


\section{Appendix C Poster Call for Participants}

This poster was distributed in print and online to call for study participants.

\section{CALL FOR PARTICIPANTS}

\section{Exercise Video Game Study}

Come and play an original game controlled by body poses!
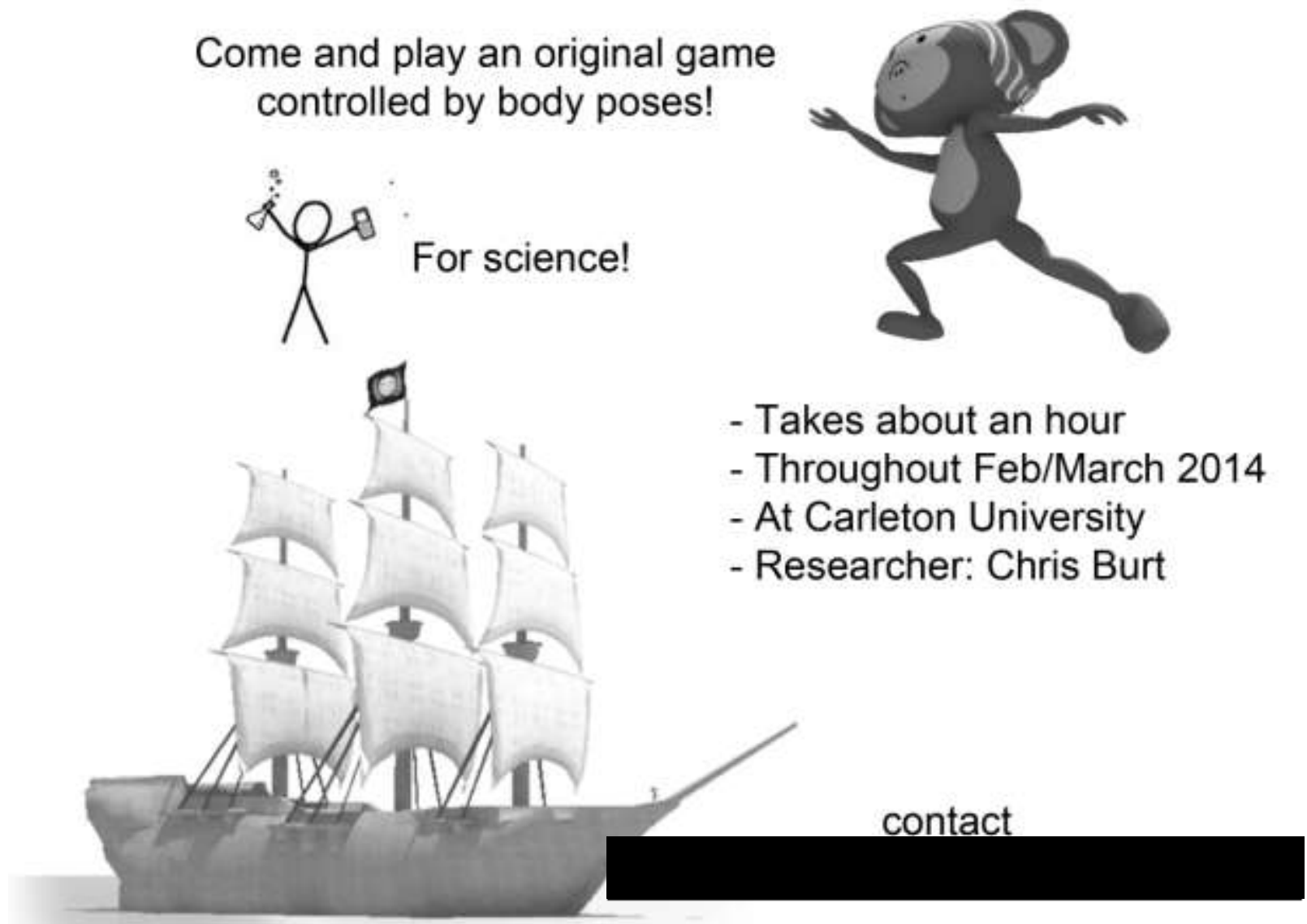


\section{Appendix D Consent Form}

This form showed approval from the Ethics department and collected participant consent.

\section{Consent Form}

Title: Improving Exercise Video Game Benefit by Adapting Challenge Based on Heart Rate

Date of ethics clearance: 12 December, 2013 (\#100729)

Ethics Clearance for the Collection of Data Expires: 31 May, 2014

I, choose to participate in a study on exercise-based video games. This study aims to evaluate the effectiveness of adapting difficulty levels. The researcher for this study is Christopher Burt in the School of Information Technology, Human-Computer Interaction.

He is working under the supervision of Anthony Whitehead in the same school.

This study involves one 60-minute session, comprised of playing the game and completing a survey. With your consent, the session will be video recorded and audio recorded.

During the session, you will wear a Zephyr HxM heart rate monitor, a small wireless device affixed about the chest with a soft elastic band. You will also wear custom-designed bodypose sensors, small wireless devices affixed about the arms and legs with velcro straps. All worn devices are non-obtrusive and require no attention or effort to wear.

During a session with the exercise video game, should the researcher become aware of potential health issues of the participant, the researcher will stop the procedure. The researcher will inform the participant that unusual readings are being noted, and ask that the participant receive written approval from a physician before continuing any participation in the study.

The data will be kept anonymous and confidential. All data will be coded such that your name is not associated with the data. All research data, including digital recordings and any notes, will be kept in a locked space at Carleton University. Only the research personnel directly involved with this experiment will have access to the data.

You have the right to end your participation in the study at any time, for any reason. You can withdraw by communicating with the researcher or the research supervisor by any means. If you withdraw from the study, all information you have provided will be immediately destroyed.

Once the project is completed, all research data will be destroyed. The approximate date of destruction is 1 April 2015. Electronic data will be erased and hard copies will be shredded.

This project was reviewed by the Carleton University Research Ethics Board, which provided clearance to carry out the research. Should you have questions or concerns related to your involvement in this research, please contact: 


\section{REB contact information:}

Professor Andy Adler, Chair

Professor Louise Heslop, Vice-Chair

Research Ethics Board

Carleton University

1325 Dunton Tower

1125 Colonel By Drive

Ottawa, ON K1S 5B6

Tel: 613-520-2517

ethics@carleton.ca

Researcher contact information:

Christopher Burt

Human-Computer Interaction

Carleton University

Tel:

Email: ChristopherBurt@cmail.carleton.ca

\section{Supervisor contact information:}

Anthony Whitehead

School of Information Technology

Carleton University

Tel:

Email: Anthony.Whitehead@carleton.ca 


\section{Appendix E Likert Response Data Summaries}

The t-test values compare the results between the Adapt group and Normal group.

\begin{tabular}{|c|c|c|c|c|c|c|c|c|c|}
\hline Question & $\begin{array}{l}\text { Mean } \\
\text { Overall }\end{array}$ & $\begin{array}{l}\text { Mean } \\
\text { Adapt }\end{array}$ & $\begin{array}{l}\text { Mean } \\
\text { Normal }\end{array}$ & $\begin{array}{l}\text { Mean } \\
\text { Male }\end{array}$ & $\begin{array}{l}\text { Mean } \\
\text { Female }\end{array}$ & $\begin{array}{l}\text { StdDev } \\
\text { Overall }\end{array}$ & $\begin{array}{l}\text { StdDev } \\
\text { Adapt }\end{array}$ & $\begin{array}{l}\text { StdDev } \\
\text { Normal }\end{array}$ & t-test \\
\hline story helped & 5.9 & 6.04 & 5.77 & 5.75 & 6.17 & 1.18 & 1.16 & 1.24 & 0.58 \\
\hline game is easy & 5.08 & 5.08 & 5.08 & 5.19 & 4.89 & 1.18 & 1.22 & 1.19 & 0.99 \\
\hline want more exercise games & 5.24 & 4.83 & 5.62 & 5.38 & 5 & 1.23 & 1.4 & 0.96 & 0.12 \\
\hline physical demand was easy & 5.38 & 5.38 & 5.38 & 5.31 & 5.5 & 1.28 & 1.13 & 1.45 & 0.99 \\
\hline aware of many exergames & 4.24 & 4 & 4.46 & 4.69 & 3.44 & 1.94 & 1.95 & 1.98 & 0.56 \\
\hline sound effects good & 5.16 & 4.58 & 5.69 & 5.25 & 5 & 1.21 & 1.16 & 1.03 & 0.02 \\
\hline cared about story & 4.96 & 5.17 & 4.77 & 4.84 & 5.17 & 1.41 & 1.32 & 1.52 & 0.49 \\
\hline game was entertaining & 5.68 & 5.5 & 5.85 & 5.72 & 5.61 & 0.73 & 0.77 & 0.69 & 0.25 \\
\hline understood characters & 6.12 & 6 & 6.23 & 6.19 & 6 & 0.86 & 0.77 & 0.95 & 0.51 \\
\hline good graphics & 4.44 & 4.33 & 4.54 & 4.5 & 4.33 & 1.64 & 1.72 & 1.61 & 0.76 \\
\hline stopped at right time & 5.12 & 4.75 & 5.46 & 5.31 & 4.78 & 1.24 & 1.06 & 1.33 & 0.15 \\
\hline poses were comfortable & 5.48 & 5.38 & 5.58 & 5.5 & 5.44 & 1.08 & 1.11 & 1.1 & 0.65 \\
\hline story was comprehendable & 6.06 & 6.21 & 5.92 & 6.13 & 5.94 & 1.05 & 0.58 & 1.37 & 0.51 \\
\hline regularly exercise & 3.88 & 4.17 & 3.62 & 3.44 & 4.67 & 2.15 & 2.41 & 1.94 & 0.53 \\
\hline game was straightforward & 6.48 & 6.46 & 6.5 & 6.5 & 6.44 & 0.65 & 0.62 & 0.71 & 0.88 \\
\hline tutorials adequate & 5.84 & 5.63 & 6.04 & 6 & 5.56 & 0.99 & 1.07 & 0.9 & 0.31 \\
\hline want more pirates & 5.32 & 5 & 5.62 & 5.31 & 5.33 & 1.55 & 1.65 & 1.45 & 0.33 \\
\hline story enhanced enjoyment & 5.72 & 5.79 & 5.65 & 5.63 & 5.89 & 1.01 & 0.72 & 1.25 & 0.74 \\
\hline could have kept going & 5.36 & 5 & 5.69 & 5.25 & 5.56 & 1.38 & 1.41 & 1.32 & 0.22 \\
\hline game was fun & 5.92 & 5.83 & 6 & 5.94 & 5.89 & 0.95 & 1.11 & 0.82 & 0.67 \\
\hline have played video games & 5.12 & 5.08 & 5.15 & 5.75 & 4 & 2.3 & 2.23 & 2.44 & 0.94 \\
\hline want sequel & 5.92 & 6.08 & 5.77 & 5.88 & 6 & 1.35 & 0.9 & 1.69 & 0.57 \\
\hline $\begin{array}{l}\text { poses understandable } \\
\text { physically became }\end{array}$ & 5.32 & 5.33 & 5.31 & 5.28 & 5.39 & 1.1 & 1.35 & 0.85 & 0.95 \\
\hline troublesome & 3.12 & 3.5 & 2.77 & 3.56 & 2.33 & 1.81 & 1.57 & 2.01 & 0.32 \\
\hline
\end{tabular}




\begin{tabular}{|c|c|c|c|c|c|c|c|c|c|c|}
\hline Question & $\begin{array}{l}\text { Mean } \\
\text { Overall }\end{array}$ & $\begin{array}{l}\text { Mean } \\
\text { Adapt }\end{array}$ & $\begin{array}{l}\text { Mean } \\
\text { Normal }\end{array}$ & $\begin{array}{l}\text { Mean } \\
\text { Male }\end{array}$ & $\begin{array}{l}\text { Mean } \\
\text { Female }\end{array}$ & $\begin{array}{l}\text { StdDev } \\
\text { Overall }\end{array}$ & $\begin{array}{l}\text { StdDev } \\
\text { Adapt }\end{array}$ & $\begin{array}{l}\text { StdDev } \\
\text { Normal }\end{array}$ & t-test & \\
\hline different story preferable & 2.76 & 2.42 & 3.08 & 3 & 2.33 & 1.48 & 1.31 & 1.61 & 0.27 & \\
\hline have played motion games & 3.04 & 2.08 & 3.92 & 3.25 & 2.67 & 2.11 & 1.24 & 2.4 & 0.03 & \\
\hline Question & $\begin{array}{l}\text { Mode } \\
\text { Overall }\end{array}$ & $\begin{array}{l}\text { Mode } \\
\text { Adapt }\end{array}$ & $\begin{array}{l}\text { Mode } \\
\text { Normal }\end{array}$ & $\begin{array}{l}\text { Median } \\
\text { Overall }\end{array}$ & $\begin{array}{l}\text { Median } \\
\text { Adapt }\end{array}$ & $\begin{array}{l}\text { Median } \\
\text { Normal }\end{array}$ & $\begin{array}{l}\text { Var } \\
\text { Overall }\end{array}$ & $\begin{array}{l}\text { Var } \\
\text { Adapt }\end{array}$ & $\begin{array}{l}\text { Var } \\
\text { Normal }\end{array}$ & f-test \\
\hline story helped & 6.5 & 6.5 & 6.5 & 6.5 & 6.5 & 6 & 1.4 & 1.34 & 1.53 & 0.84 \\
\hline game is easy & 5.5 & 3.5 & 5.5 & 5.5 & 5.25 & 5.5 & 1.39 & 1.49 & 1.41 & 0.92 \\
\hline want more exercise games & 6 & 5 & 6 & 5 & 5 & 6 & 1.52 & 1.97 & 0.92 & 0.21 \\
\hline physical demand was easy & 6.5 & 4 & 6.5 & 5.5 & 5.25 & 6 & 1.63 & 1.28 & 2.09 & 0.42 \\
\hline aware of many exergames & 5 & 5 & 6 & 5 & 4 & 5 & 3.77 & 3.82 & 3.94 & 0.97 \\
\hline sound effects good & 5 & 5 & 5 & 5 & 5 & 5 & 1.47 & 1.36 & 1.06 & 0.68 \\
\hline cared about story & 5 & 4.5 & 5 & 5 & 5 & 5 & 2 & 1.74 & 2.32 & 0.64 \\
\hline game was entertaining & 5.5 & 6 & 6.5 & 5.5 & 5.5 & 5.5 & 0.54 & 0.59 & 0.47 & 0.71 \\
\hline understood characters & 6.5 & 6 & 6.5 & 6.5 & 6 & 6.5 & 0.73 & 0.59 & 0.9 & 0.49 \\
\hline good graphics & 6 & 6 & 6 & 5 & 5 & 5 & 2.67 & 2.97 & 2.6 & 0.82 \\
\hline stopped at right time & 6 & 5 & 6 & 5 & 5 & 6 & 1.53 & 1.11 & 1.77 & 0.45 \\
\hline poses were comfortable & 6 & 5 & 6 & 5.5 & 5.25 & 6 & 1.18 & 1.23 & 1.2 & 0.96 \\
\hline story was comprehendable & 7 & 6 & 7 & 6.5 & 6 & 6.5 & 1.11 & 0.34 & 1.87 & 0.01 \\
\hline regularly exercise & 1 & 1 & 1 & 4 & 5 & 4 & 4.61 & 5.79 & 3.76 & 0.47 \\
\hline game was straightforward & 7 & 7 & 7 & 6.5 & 6.5 & 6.5 & 0.43 & 0.38 & 0.5 & 0.67 \\
\hline tutorials adequate & 6.5 & 6 & 6.5 & 6 & 6 & 6.5 & 0.97 & 1.14 & 0.81 & 0.56 \\
\hline want more pirates & 7 & 4 & 7 & 6 & 4 & 6 & 2.39 & 2.73 & 2.09 & 0.65 \\
\hline story enhanced enjoyment & 6 & 6 & 6 & 6 & 6 & 6 & 1.02 & 0.52 & 1.56 & 0.08 \\
\hline could have kept going & 5 & 5 & 6 & 5 & 5 & 6 & 1.91 & 2 & 1.73 & 0.8 \\
\hline game was fun & 6 & 6 & 6 & 6 & 6 & 6 & 0.91 & 1.24 & 0.67 & 0.3 \\
\hline have played video games & 7 & 7 & 7 & 6 & 6 & 7 & 5.28 & 4.99 & 5.97 & 0.77 \\
\hline want sequel & 7 & 7 & 7 & 6 & 6 & 6 & 1.83 & 0.81 & 2.86 & 0.05 \\
\hline poses understandable & 5.5 & 5.5 & 6.5 & 5.5 & 5.5 & 5 & 1.21 & 1.83 & 0.73 & 0.13 \\
\hline
\end{tabular}




\begin{tabular}{|c|c|c|c|c|c|c|c|c|c|c|}
\hline Question & $\begin{array}{l}\text { Mode } \\
\text { Overall }\end{array}$ & $\begin{array}{l}\text { Mode } \\
\text { Adapt }\end{array}$ & $\begin{array}{l}\text { Mode } \\
\text { Normal }\end{array}$ & $\begin{array}{l}\text { Median } \\
\text { Overall }\end{array}$ & $\begin{array}{l}\text { Median } \\
\text { Adapt }\end{array}$ & $\begin{array}{l}\text { Median } \\
\text { Normal }\end{array}$ & $\begin{array}{l}\text { Var } \\
\text { Overall }\end{array}$ & $\begin{array}{l}\text { Var } \\
\text { Adapt }\end{array}$ & $\begin{array}{l}\text { Var } \\
\text { Normal }\end{array}$ & f-test \\
\hline $\begin{array}{l}\text { physically became } \\
\text { troublesome }\end{array}$ & 1 & 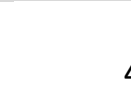 & 1 & 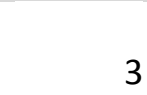 & 3.5 & te & 3.28 & 2.45 & 4.03 & 0.42 \\
\hline different story preferable & 4 & 2 & 4 & 3 & 2 & 3 & 2.19 & 1.72 & 2.58 & 0.51 \\
\hline have played motion games & 1 & & 1 & 2 & 2 & 5 & 4.46 & 1.54 & 5.74 & 0.04 \\
\hline
\end{tabular}


Responses regarding psychological flow components represent the averages of Flow State Scale questions pertaining to each component.

\begin{tabular}{|c|c|c|c|c|c|c|c|c|c|}
\hline Question & $\begin{array}{l}\text { Mean } \\
\text { Overall }\end{array}$ & $\begin{array}{l}\text { Mean } \\
\text { Adapt }\end{array}$ & $\begin{array}{l}\text { Mean } \\
\text { Normal }\end{array}$ & $\begin{array}{l}\text { Mean } \\
\text { Male }\end{array}$ & $\begin{array}{l}\text { Mean } \\
\text { Female }\end{array}$ & $\begin{array}{l}\text { StdDev } \\
\text { Overall }\end{array}$ & $\begin{array}{l}\text { StdDev } \\
\text { Adapt }\end{array}$ & $\begin{array}{l}\text { StdDev } \\
\text { Normal }\end{array}$ & t-test \\
\hline flow ENJY & 5.32 & 4.94 & 5.67 & 5.45 & 5.08 & 0.95 & 0.97 & 0.81 & 0.05 \\
\hline flow GOAL & 5.86 & 5.92 & 5.81 & 5.98 & 5.64 & 0.86 & 0.71 & 1 & 0.76 \\
\hline flow CHAL & 5.56 & 5.67 & 5.46 & 5.53 & 5.61 & 0.63 & 0.7 & 0.56 & 0.42 \\
\hline flow CONC & 5.33 & 5.48 & 5.19 & 5.25 & 5.47 & 0.91 & 0.86 & 0.96 & 0.44 \\
\hline flow CONT & 5.4 & 5.29 & 5.5 & 5.34 & 5.5 & 0.8 & 0.76 & 0.85 & 0.53 \\
\hline flow FDBK & 5.58 & 5.08 & 6.04 & 5.52 & 5.69 & 1.02 & 1.08 & 0.73 & 0.02 \\
\hline flow ACT & 5.29 & 5 & 5.56 & 5.44 & 5.03 & 0.83 & 0.79 & 0.79 & 0.09 \\
\hline flow TRAN & 3.9 & 3.71 & 4.08 & 3.89 & 3.92 & 1.13 & 1.08 & 1.19 & 0.43 \\
\hline $\begin{array}{l}\text { flow LOSS } \\
\text { FLOW }\end{array}$ & 4.69 & 4.44 & 4.92 & 4.81 & 4.47 & 1.67 & 1.73 & 1.65 & 0.48 \\
\hline OVERALL & 5.21 & 5.06 & 5.36 & 5.25 & 5.16 & 0.5 & 0.5 & 0.48 & 0.14 \\
\hline
\end{tabular}

\begin{tabular}{|c|c|c|c|c|c|c|c|c|c|c|}
\hline Question & $\begin{array}{l}\text { Mode } \\
\text { Overall }\end{array}$ & $\begin{array}{l}\text { Mode } \\
\text { Adapt }\end{array}$ & $\begin{array}{l}\text { Mode } \\
\text { Normal }\end{array}$ & $\begin{array}{l}\text { Median } \\
\text { Overall }\end{array}$ & $\begin{array}{l}\text { Median } \\
\text { Adapt }\end{array}$ & $\begin{array}{l}\text { Median } \\
\text { Normal }\end{array}$ & $\begin{array}{l}\text { Var } \\
\text { Overall }\end{array}$ & $\begin{array}{l}\text { Var } \\
\text { Adapt }\end{array}$ & $\begin{array}{l}\text { Var } \\
\text { Normal }\end{array}$ & f-test \\
\hline flow ENJY & 6 & 4 & 6 & 5.25 & 5.13 & 6 & 0.9 & 0.93 & 0.66 & 0.56 \\
\hline flow GOAL & 5.75 & 5.75 & 6.25 & 6 & 5.88 & 6 & 0.73 & 0.5 & 1 & 0.27 \\
\hline flow CHAL & 5.5 & 5.5 & 5.25 & 5.5 & 5.63 & 5.25 & 0.39 & 0.49 & 0.31 & 0.44 \\
\hline flow CONC & 5 & 5.75 & 5 & 5.5 & 5.75 & 5.25 & 0.83 & 0.74 & 0.93 & 0.72 \\
\hline flow CONT & 5.25 & 4.5 & 5.25 & 5.25 & 5.13 & 5.5 & 0.64 & 0.58 & 0.73 & 0.71 \\
\hline flow FDBK & 5.75 & 5.75 & 6.75 & 5.75 & 5.25 & 6 & 1.04 & 1.16 & 0.53 & 0.19 \\
\hline flow ACT & 5.25 & 5 & 6 & 5.25 & 5.13 & 5.5 & 0.68 & 0.63 & 0.63 & 1 \\
\hline flow TRAN & 3.25 & 3.25 & 3.5 & 4 & 3.88 & 4 & 1.27 & 1.16 & 1.41 & 0.75 \\
\hline flow LOSS & 6 & 5.5 & 6 & 5.25 & 5 & 5.25 & 2.8 & 2.99 & 2.73 & 0.87 \\
\hline \multicolumn{11}{|l|}{ FLOW } \\
\hline OVERALL & 5.53 & 4.89 & $\mathrm{n} / \mathrm{a}$ & 5.31 & 4.94 & 5.47 & 0.25 & 0.25 & 0.23 & 0.86 \\
\hline
\end{tabular}

